Aus der Abteilung Anaesthesiologie II - Operative Intensivmedizin (Ehem. Leiter: Prof. Dr. med. H. Burchardi) im Zentrum Anaesthesiologie, Rettungs- und Intensivmedizin der Medizinischen Fakultät der Universität Göttingen

\title{
Polysomnographie auf der Intensivstation
}

Eine Untersuchung des Schlafes von Patienten einer Intensivstation der Universität Göttingen mit Hilfe der PSG

\author{
INAUGURAL - DiSSERTATION \\ zur Erlangung des Doktorgrades \\ der Medizinischen Fakultät \\ der Georg-August-Universität zu Göttingen \\ vorgelegt von Thomas Moritz Engels \\ aus Memmingen
}

Göttingen 2010 
Dekan:

I. Berichterstatter:

II. Berichterstatter/in:

III. Berichterstatter/in:

Tag der mündlichen Prüfung:
Prof. Dr. med. C. Frömmel

Prof. Dr. med. H. Burchardi 


\section{Inhaltsverzeichnis}

1 Einleitung 3

1.1 Der normale menschliche Schlaf . . . . . . . . . . . . . . . 3

1.2 Der Schlaf auf der Intensivstation . . . . . . . . . . . . . . . . 9

1.3 Zielsetzung \& Fragestellung . . . . . . . . . . . . . . . . 17

2 Methoden $\quad 18$

$2.1 \quad$ Studienart . . . . . . . . . . . . . . . . . . . . . . 18

2.2 Studienort . . . . . . . . . . . . . . . . . . . . . . . . . . . . 18

2.3 Studienpopulation . . . . . . . . . . . . . . . . . . . . . . . . 19

2.4 Studienablauf . . . . . . . . . . . . . . . . . . . 21

2.5 Untersuchungsdurchführung . . . . . . . . . . . . . . . . . . 24

2.6 Schlafstadienanalyse, Schlafparameter, Arousal . . . . . . . . . . 28

2.7 Prüfung der Messungen . . . . . . . . . . . . . . . . . . . . . 32

2.8 Auswertung . . . . . . . . . . . . . . . . . . 35

$\begin{array}{lll}3 & \text { Ergebnisse } & 40\end{array}$

3.1 Die Population . . . . . . . . . . . . . . . . . . . . . . . 40

3.2 Auswertung . . . . . . . . . . . . . . . . . . . . 43

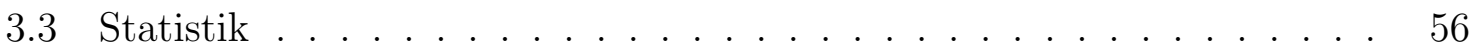

4 Diskussion $\quad 60$

4.1 Studienort und Studienplanung . . . . . . . . . . . . . . 60 
4.2 Population . . . . . . . . . . . . . . . . . . . . 61

4.3 Untersuchungsverfahren . . . . . . . . . . . . . . . . . . . . . 62

4.4 Auswertung . . . . . . . . . . . . . . . . . . . . . . . 64

4.5 Ergebnisse . . . . . . . . . . . . . . . . . . . . . 64

4.6 Statistik . . . . . . . . . . . . . . . . . 76

4.7 Resumee . . . . . . . . . . . . . . . . . . . 76

5 Zusammenfassung $\quad 80$

6 Anhang $\quad 82$

6.1 Abkürzungsverzeichnis . . . . . . . . . . . . . . . . . . . . . . 82

6.2 Abbildungen und Tabellen . . . . . . . . . . . . . . . . . 84

8 Abbildungsverzeichnis $\quad 96$

9 Tabellenverzeichnis $\quad 98$ 


\section{Kapitel 1}

\section{Einleitung}

\subsection{Der normale menschliche Schlaf}

Definition: "Schlaf, durch Änderungen des Bewusstseins, entspannte Ruhelage und Umstellung verschiedener vegetativer Körperfunktionen gekennzeichneter Erholungsvorgang des Gesamtorganismus, vor allem des Zentralnervensystems, der von einer inneren, mit dem Tag-Nacht Wechsel synchronisierten Periodik gesteuert wird; dabei kommt es zu einem Verlust der räumlichen, zeitlichen und personellen Orientierung durch eine vom Stammhirn verursachte Funktionsänderung der Hirnrinde. Ebenso wie die Aufmerksamkeit im Wachen variieren kann, ändert sich auch die Schlaftiefe, kenntlich an der Stärke des zur Unterbrechung des Schlafes erforderlichen Weckreizes." (Brockhaus 2004 S.4197)

Schlaf ist kein statischer Zustand des Zentralnervensystems. Vielmehr verändern sich die Hirnfunktionen in zyklisch aufeinander folgenden Zeitabschnitten (sogenannte Schlafzyklen) während einer Schlafperiode (z.B. im Laufe einer Nacht). Der Schlaf kann in zwei verschiedene Grundzustände aufgeteilt werden, und zwar den Non-Rapid-EyeMovement-Schlaf (NREM-Schlaf) und den Rapid-Eye-Movement-Schlaf (REM-Schlaf). 
Der Schlafzyklus beginnt mit dem NREM-Schlaf, der wiederum in 4 Stadien S1 bis S4 eingeteilt wird, und geht dann in den REM-Schlaf über. Die Stadien S1-S4 sind durch ansteigende Schlaftiefe, und ein eigenes charakteristisches EEG-Muster gekennzeichnet. Im Stadium S1 kann der Schlafende am leichtesten erweckt werden und im Schlafstadium S4 am schwersten. Somit ist die akustische Arousalschwelle (AAT), das heißt die Weckreaktion auf einen akustischen Reiz, im Stadium S1 am niedrigsten und im S4 am höchsten (Ferrara et al. 1999). Das Wachstadium ist durch die höchste Alpha-Aktivität und hohen submentalen Muskeltonus geprägt. Für die Schlafstadien S1 und S2 ist ein schnelles hochfrequentes EEG mit niedriger Amplitude charakteristisch.

Im Stadium S2 ist das EEG etwas niederfrequenter als im Stadium S1 und weist sogenannte Spindeln und K-Komplexe auf. Spindeln werden ihrem Aussehen entsprechend benannt, sie haben Frequenzen um 12-14 Hz bei mittlerer Amplitude und dauern länger als 0,5 Sekunden an. Die K-Komplexe hingegen sind gekennzeichnet durch eine schnell negative Amplitude mit nachfolgend langsam ansteigender, positiver Komponente. Die Stadien S3 und S4 werden auch als Deltaschlaf bezeichnet und sind charakterisiert durch langsame Wellen mit großen Amplituden, die $\delta$-wellen von circa 1-2 Hz und einer Amplitude über $75 \mathrm{mV}$. Stadium S4 unterscheidet sich lediglich durch vermehrte $\delta$-wellen von Stadium S3. Das Merkmal des REM-Schlafes ist ein hochfrequentes EEG mit sogenannten Sägezahnwellen, schnellen, gleichlaufenden Augenbewegungen und einem sehr niedrigen Muskeltonus.

Der Aufbau einer Schlafperiode wird Schlafarchitektur genannt. Eine Schlafperiode umfasst in einer 8-stündigen Schlafperiode 5-6 Schlafzyklen. Dabei ist innerhalb eines Zyklus ein rhythmischer Wechsel vom NREM-Schlaf zum REM-Schlaf zu beobachten, welcher alle 90 bis 110 Minuten auftritt. Typischerweise tritt der REM-Schlaf in den letzten 10-30 Minuten eines Zyklus auf. Der REM-Schlafanteil nimmt im Laufe einer Nacht zu, analog dazu nimmt der Anteil des NREM-Schlafs ab (Carskadon und Dement 2000, Rechtschaffen und Kales 1968, Schwab 1994).

In Abbildung 1.1 ist die Aufteilung der Schlafstadien eines gesunden Probanden nach Carskadon und Dement sowie Steinberg et al. aus dem Jahre 2000 wiedergegeben. 
Abbildung 1.1: Verteilungsmuster der Schlafstadien eines Gesunden nach Carskadon und Dement (2000) sowie Steinberg et al. (2000)

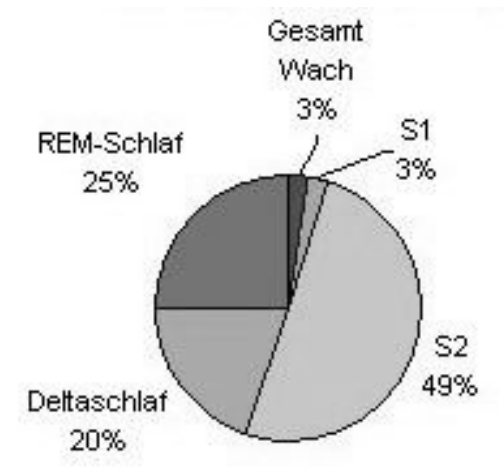

\begin{tabular}{|l|}
\hline Gesamt \\
Wach \\
$\square S 1$ \\
$\square S 2$ \\
$\square$ Deltaschlaf \\
$\square$ REM- \\
Schlaf \\
\hline
\end{tabular}

\subsubsection{Historischer Überblick}

Die Technik der Polysomnographie (PSG) wurde in den Schriften von Loomis et al. von 1935 und 1937 erstmals eingeführt. Er erkannte, dass die hirnelektrische Aktivität mit zunehmender Tiefe des Non-Rapid-Eye-Movement-Schlafs (NREM-Schlaf) kontinuierlich abnimmt. Dement und Kleitman (1957a,b) beschrieben den REM-Schlaf als periodische Phasen rascher Augenbewegungen, die von hochfrequenten und niedrigamplitudigen Hirnströmen begleitet werden. 1968 standardisierten Rechtschaffen und Kales in ihrem Manual die Terminologie, Techniken und Aufnahmesysteme für verschiedene Schlafstadien am Menschen. Neueste Techniken erlauben es, die PSG digital aufzunehmen und eine große Menge an Daten über den Schlaf zu gewinnen. Somit besteht die Möglichkeit, mittels PSG, Aussagen über Qualität und Quantität des menschlichen Schlafs zu treffen. Es entstanden viele Studien die sich mit dem Schlaf kranker und schwer kranker Menschen beschäftigen. Dass der Schlaf der Patienten von dem eines Gesunden stark abweicht, ist leicht vorstellbar. Die Ursachen, die zu diesen Veränderungen führen, sind unzulänglich geklärt. Patienten einer Intensivstation 
(Intensiv-Care-Unit; ICU) weisen häufig einen stark von der Norm abweichenden Schlaf auf. Es gibt verschiedene Ursachen, wie z.B. Lärm, Therapie, Diagnostik oder Waschen und Körperpflege, die negativen Einfluss auf den Schlaf ausüben, die bisher nicht verringert werden konnten. Das junge und weite Feld der Somnologie ist noch ungenügend erforscht. Noch viele Studien und Experimente werden nötig sein, um die vielfachen Faktoren, die auf den Schlaf und im Speziellen auf den Schlaf der Patienten in intensivmedizinischer Behandlung wirken, zu bestimmen und ihre Auswirkungen einschätzen zu können. Träume kommen im Schlaf und helfen uns das Erlebte zu verarbeiten. Man sagt den Träumen und dem Schlaf eine heilende Wirkung nach. Diese Untersuchung soll ein Beitrag dazu sein, dass der Schlaf und dessen heilende Wirkung im Krankenhaus, speziell auf der Intensivstation, wieder Einzug hält.

\subsubsection{Das Arousal}

Es kann vorkommen, dass der Schlaf durch Weckreaktionen, sogenannte Arousals, unterbrochen wird. Ein Arousal kann zu einer partiellen, temporären oder vollständigen Weckreaktion führen. Es hat auf den Schlaf stets eine unterbrechende Wirkung und stellt für viele Schlafstörungen einen wesentlichen Pathomechanismus dar. Bereits im diagnostischen Manual von Rechtschaffen und Kales (1968) werden Arousalreaktionen berücksichtigt. Für die klinische Schlafmedizin sind diese Kriterien jedoch nicht ausreichend gewesen. Deshalb entwickelte 1997 die American Sleep Disorders Association (ASDA) eine EEG-bezogene Arousal-Klassifikation. Ihr liegen die klassischen Ableitungen von Rechtschaffen und Kales (1968) zugrunde. Per definitionem wird unter einem Arousal eine abrupte Frequenzänderung oder Frequenzbeschleunigung im EEG verstanden. Sie beinhaltet Theta-Wellen, Alpha-Wellen oder Frequenzen über 16 Hz. Schlafspindeln mit ihren charakteristischen Frequenzen zwischen 12 und $14 \mathrm{~Hz}$ sind ausgenommen.

Steinberg et al. (2000) beschreiben Arousals als 'organische Schutzfunktionen', die nur pathologisch sind, wenn sie gehäuft auftreten. Sie seien eine Reaktion auf bedrohli- 
che Umstände z.B. aus der Umwelt wie Feuer oder Kälte oder auf andere bedrohliche Zustände des Körpers wie Atemstillstand, kardiale Ischämien oder angsterregende Trauminhalte. Die Ursachen können vielfältig sein und werden hauptsächlich in enterozeptive (psychophysisch, sensorisch oder neuronal) oder exterozeptive (akustisch, optisch, taktil, olfaktorisch oder thermisch) unterteilt (Steinberg et al. 2000 S.147). Die Weckreaktionen insbesondere die akustisch bedingten Arousalreaktionen sind für schlafmedizinische Fragestellungen in der modernen Intensivmedizin von entscheidender Bedeutung, da der Schallpegel auf einer Intensivstation im Vergleich zu Normalbedingungen erhöht ist (Freedman et al. 2001, Schwab 1994, Topf 1992).

Die Auslöser eines Arousals sind von unterschiedlichster Art. Lärm, Licht, Manipulation oder Schmerz können ein Arousal auslösen, aber auch spezielle Erkrankungen, wie zum Beispiel das Schlafapnoesyndrom (SAS) führen nach Sanner (1992) zu einem gehäuften Auftreten von Arousals. Ein Schlafzyklus dauert 90 bis 110 Minuten. Laut Carola et al. (1990) und Wood (1993) durchlaufen Patienten, die während eines Zyklus durch ein Arousal, gleich welcher Art, gestört wurden, erneut, beginnend bei Stadium S1, die komplette Schlafperiode.

\subsubsection{Arousalklassen}

Die Standard-Arousals entsprechen der modernen Schlafmedizin und werden in Tabelle 1.1 (S.8) definiert. Die verschiedenen Arousals erlauben eine qualitative Aussage hinsichtlich der Entstehung, um somit eine Diagnose wie zum Beispiel SAS stellen zu können. Da diese Untersuchung auf der Intensivstation stattfand, musste die Arousalklassifikation um zusätzliche Einflüsse, die beim Normalschlaf nicht vorkommen, erweitert werden. Es existiert hier eine Mehrzahl zusätzlicher Einflüsse, die ein Arousal hervorrufen können. Darunter sind in erster Linie externe Einflüsse zu nennen, die von besonderer Bedeutung sind und mit der hier beschriebenen Methodik auch erfasst und analysiert werden können. Besonders werden akustische Störungen berücksichtigt, da diese häufiger zu erwarten waren als die taktilen. Da es sich um spezifische Arousals einer Intensivstation (ICU) handelt, die im normalen Schlaf nicht vorkommen, werden 
Tabelle 1.1: Zusammenfassende Definition der Standard-Arousals nach Stein-

berg et al. 2000 (S.44-55 und S.145-148)

\begin{tabular}{|c|c|c|}
\hline Arousal & Abkürzung & Definition \\
\hline Spontan & A: & $\begin{array}{l}\text { Arousal ohne erkennbaren Grund; in keinem Kanal } \\
\text { ersichtliches Ereignis vorausgegangen. }\end{array}$ \\
\hline Extremitätenbewegung & A:LM & $\begin{array}{l}\text { Bewegungsassoziiertes Arousal; } 0,5 \text { bis } 5 \text { sec Bewe- } \\
\text { gung vor dem Arousal mit Amplitude mind. } 25 \mathrm{mV}\end{array}$ \\
\hline Period. Extremitätenbewegung & $\mathrm{A}: \mathrm{PLM}$ & $\begin{array}{l}\text { Vor Arousal mindestens } 4 \text { eindeutige periodisch wie- } \\
\text { derkehrende Beinbewegungen. Intervall zwischen Be- } \\
\text { wegungen einer Sequenz } 5 \text { bis } 90 \mathrm{sec} \text {. }\end{array}$ \\
\hline Resp. Extremitätenbewegung & A:RRLM & $\begin{array}{l}\text { Zeitlicher Zusammenhang zwischen respiratorischen } \\
\text { Ereignissen (Apnoe o. Hypopnoe) und Extremitäten- } \\
\text { bewegungen. }\end{array}$ \\
\hline \multicolumn{3}{|l|}{ Auf die Atmung bezogene Arousals: } \\
\hline Apnoe & $\mathrm{A}: \mathrm{APN}$ & $\begin{array}{l}\text { Arousal mit mind. 90\% Verminderung des Luftflusses } \\
\text { wenigstens 10sec vor Arousal. }\end{array}$ \\
\hline Zentrale Apnoe & $\mathrm{A}: \mathrm{CA}$ & $\begin{array}{l}\text { Siehe A:APN. Es darf keine Obstruktion der Atem- } \\
\text { wege vorliegen. Thorax-, Abdomen- und Atem- } \\
\text { flusskurve weisen gleichermaßen einen um mehr als } \\
90 \% \text { verminderten Ausschlag auf. }\end{array}$ \\
\hline Obstruktive Apnoe & $\mathrm{A}: \mathrm{OA}$ & $\begin{array}{l}\text { Im Unterschied zur zentralen Apnoe ist hier eine } \\
\text { Atembewegung des Thorax und Abdomen zu erken- } \\
\text { nen. Der Flow ist mehr als } 90 \% \text { vermindert. }\end{array}$ \\
\hline Gemischte Apnoe & $\mathrm{A}: \mathrm{GA}$ & $\begin{array}{l}\text { Mischung der obstruktiven und zentralen Kompo- } \\
\text { nenten. }\end{array}$ \\
\hline Hypopnoe & $\mathrm{A}: \mathrm{HYPN}$ & $\begin{array}{l}\text { Arousal mit mind. } 50 \% \text { Verminderung des Luftflusses } \\
\text { wenigstens } 10 \mathrm{sec} \text { vor Arousal. }\end{array}$ \\
\hline Zentrale Hypopnoe & $\mathrm{A}: \mathrm{CH}$ & $\begin{array}{l}\text { Siehe A:HYPN. Hinzu kommt, dass keine Obstrukti- } \\
\text { on der Atemwege vorliegt. Thorax-, Abdomen- und } \\
\text { Atemflusskurve gleichermaßen um mehr als } 50 \% \text { ver- } \\
\text { minderter Ausschlag. }\end{array}$ \\
\hline Obstruktive Hypopnoe & $\mathrm{A}: \mathrm{OH}$ & $\begin{array}{l}\text { Atembewegung von Thorax und Abdomen zu erken- } \\
\text { nen. Der Flow ist mehr als } 50 \% \text { vermindert. }\end{array}$ \\
\hline Gemischte Hypopnoe & $\mathrm{A}: \mathrm{GH}$ & $\begin{array}{l}\text { Mischung der obstruktiven und zentralen Kompo- } \\
\text { nenten. }\end{array}$ \\
\hline Entsättigung & A:DESAT & Abfall $>5 \%$ der momentanen Sättigung vor Arousal. \\
\hline Schnarchen & A:SNORE & $\begin{array}{l}\text { Schnarch-bedingtes Arousal. Dem Arousal geht ein } \\
\text { Schnarchereignis voraus. Amplitude: über } 20 \mathrm{mV} \text {. } \\
\text { Dauer: Maximal 10, minimal 3sec. Unterbrechung } \\
\text { des Schnarchens < 1sec. minimale Frequenz: } \sim 10 \mathrm{~Hz} \text {. }\end{array}$ \\
\hline
\end{tabular}

Wir beschränkten uns auf die Standard-Arousals auf A:; A:LM; A:PLM; A:APN und A:HYPN und A.SNORE. Period.: Periodisch; Resp.: Respiratorisch 
diese im Folgenden Intensiv-Care-Unit-Arousals oder kurz ICU-Arousals genannt.

\subsection{Der Schlaf auf der Intensivstation}

Auf einer Intensivstation befinden sich vorwiegend Patienten, deren Vitalfunktionen bedroht sind, oder aufrecht erhalten werden müssen, oder postoperativ bedingt einer intensiven Überwachung bedürfen. Wood (1993) konnte zeigen, dass Schlaf ein entscheidender Faktor ist, der zur Erholung und Genesung von Patienten beiträgt. Helton et al. zeigten 1980 in ihren Untersuchungen, dass der Schlaf eines Intensivpatienten, wenn er Ruhe am meisten benötigt, bis zu 14 mal pro Stunde unterbrochen wurde. In der Literatur werden sogenannte Schlafparameter verwendet, die versuchen den Schlaf der Patienten quantitativ oder qualitativ zu beschreiben. Sie können mit Hilfe der PSG bestimmt werden. Definition und Erläuterung siehe Tabelle 2.3 (S.30), orientierende Kennwerte in Tabelle 4.1 (S.66). Die Schlafparameter eines Patienten in intensivmedizinischer Behandlung zeigen verglichen mit den Schlafparametern eines Gesunden starke Veränderungen. In der Literatur gibt es darüber weitreichende Übereinstimmungen wie z.B. bei den Autoren Rosenberg et al. (1995), Schwab (1994) und Steinberg et al. (2000). Hauptsächlich verlängert sich die Einschlaflatenz. Schlafdauer, Totale Schlafzeit, Schlafeffizienz und -kontinuität verringern sich, der $\delta$ - und REM-Schlaf verringern sich zu Gunsten der leichten Schlafstadien S1 und S2. Diese Befunde wurden in Tabelle 6.1 zusammengefasst und sind im Anhang auf Seite 85 einzusehen.

Die Gründe der Schlafunterbrechungen sind unter anderem in der intensiven Überwachung zu suchen. Es ist nötig, dass die Vitalparameter intensivmedizinisch betreuter Patienten auch nachts engmaschig kontrolliert werden. Eine Vielzahl an pflegerischen Maßnahmen, diagnostischen und therapeutischen Interventionen wie z.B. Röntgenuntersuchungen, unterstützende Beatmung, Blutabnahme, Visite, Verbands- und Katheterkontrolle, Physiotherapie, Pneumonieprophylaxe sowie Lagerung und Körperpflege tragen dazu bei, den Schlaf und die Ruhe des Patienten empfindlich zu stören. Mechanische Beatmung und ein endotrachealer Tubus stören laut der Untersuchung von Calvete 
Vazquez et al. (2000) den Schlaf am meisten. Auch das oben genannte Schlafapnoesyndrom kann durch die nachfolgende Hypopnoe oder Apnoe eine hypoxisch bedingte Weckreaktion hervorrufen. Als Auslöser und Verstärker des SAS seien hier z.B. Medikamente genannt, die auf die Atmung und den Muskeltonus depressiv wirken. Hier beginnt der Übergang zu den intrinsichen Einflüssen, denn auch innere Einflüsse des Patienten führen zu Veränderungen der Schlafarchitektur. Jede Anspannung oder Ausschüttung von Adrenalin und anderen Stresshormonen bedingt eine Beeinträchtigung des Schlafes. Stressreaktionen des Körpers werden psychisch durch vielerlei Faktoren getriggert. $\mathrm{Zu}$ nennen sind unter anderem Schmerzen, Furcht und Angst, Ungewissheit, verminderter Kontakt zur Außenwelt, psychosoziale Faktoren, Postagressionsstoffwechsel, Medikamente oder Fieber (Calvete Vazquez et al. 2000).

Wie bereits erwähnt können Arousals extrinsisch oder intrinsisch ausgelöst werden. Schwab untersuchte die Einflüsse auf intensiv überwachte Patienten und berücksichtigte auch den Schallpegel. Er differenzierte dabei zwischen verschiedenen Lärmquellen und gab zusätzlich die Höhe der Lärmbelastung an (Schwab 1994).

Alle in Tabelle 1.2 genannten Faktoren können die oben genannten Schlafparameter negativ beeinflussen oder können ein Arousal hervorrufen. Mit Hilfe der PSG können Schlafparameter, Arousals und Weckreaktionen auch bei intensivmedizinisch behandelten Patienten sichtbar gemacht werden. Aufgrund der methodischen Einschränkungen können nur die extrinsischen Einflüsse beurteilt werden. Daher werden im folgenden Kapitel speziell die akustischen Einflüsse behandelt.

\subsubsection{Akustische Einflüsse auf Arousals}

Für den gesunden Menschen ist Schlaf unter Lärmbelästigung qualitativ nicht hochwertig und hat somit nur einen geringen Erholungswert. Die meisten gesunden Probanden, die unter Lärmbedingungen schlafen mussten, gaben laut Topf et al. (1995) an, schlecht oder wenig geschlafen zu haben. Patienten einer Intensivstation sind diesen äußeren Einflüssen zusätzlich ausgesetzt. Auch Nachts sind Schwestern und Ärzte 


\section{Tabelle 1.2: Intrinsische und extrinsische Faktoren auf ICU}

1. Intrinsische Faktoren

- Schwere der Krankheit

- Medikation

- REM unterdrückende Stoffe

* Narkotika, Barbiturate, Antidepressiva

- NREM unterdrückende Stoffe

* Benzodiazepine

- Schmerz

- Fieber

- Kontrollverlust (Einschränkung, pharmakologische Paralyse)

- Furcht, Angst, psychosozialer Stress

2. Extrinsische Faktoren

- Arzt/ Pflege

- Diagnostische Verfahren

- Pflege-bedingte Interventionen

- Invasive Prozeduren

- Umweltfaktoren

- Licht

- Lärm

* Geräte inklusive Beatmung und Alarme (45-76 dB)

* Hintergrundgeräusch (55-72 dB)

* Pflege oder respiratory care (respiratorische Pflege) (55-83 dB); darunter fallen das Beatmungsgerät selbst, Absaugen und andere mit der respiratorischen Pflege zusammenhängende Tätigkeiten.

* Konversation von Ärzten und Personal untereinander (60-74 dB)

- Reizende Gerüche

Intrinsische und extrinsische Faktoren eines Schlafenden Patienten einer ICU - modifiziert nach Schwab 1994 (S.686). 
durchgehend auf der Station tätig und erzeugen durch ihre Arbeit Lärm. Eine Vielzahl an intensivmedizinischen Geräten wie Monitore, Perfusoren oder Beatmungsgeräte tragen dazu bei, den Schallpegel drastisch zu erhöhen. Calvete Vazquez et al. stellten in ihrer Untersuchung im Jahre 2000 fest, dass die Alarmsignale der medizinischen Geräte am meisten stören. Auch Wood benannte 1993 Lärm auf einer Intensivstation als den größten Störfaktor, wobei der Patient besonders durch die Kommunikation des Personals untereinander gestört wurde.

Bentley et al. gingen 1977 einen Schritt weiter und definierten eine maximale Schallschwelle von $30 \mathrm{~dB}$, die nicht überschritten werden dürfe, um erholsamen Schlaf oder Schlaf generell zu ermöglichen. In den Untersuchungen Bentleys et al. wurde festgestellt, dass dieser Richtwert auf einer Intensivstation deutlich überschritten wird. Auch Schwab setzte 1994 eine obere Schallgrenze von 35 bis 40 dB, die notwendig sei, damit ein Patient ungestört einschlafen könne. Überschreitungen dieser Grenze führten zu vermehrten Arousals.

In einer Studie Aarons wurde der Lärm einer Intensivstation mit den Schlafunterbrechungen eines Schlafenden verglichen. Er fand heraus, dass in einer bestimmten Zeit, in der vermehrt Schallspitzen größer als 80 dB auftraten, auch vermehrt Arousals auftraten. Aufgrund der hohen Signifikanz zwischen Arousals und Schallhöchstwerten über $80 \mathrm{~dB}$ folgerte er, dass viele Patienten aufgrund eines erhöhten Schallpegels an Schlafentzug leiden (Aaron et al. 1996).

Es existiert eine weitere Untersuchung von Freedman et al. (2001), in der erstmals Patienten zeitgleich mit PSG und Schallpegelmessung überwacht wurden. Dabei stellten Freedman et al. einen direkten Zusammenhang zwischen Lärm und Arousal her. In deren Untersuchung wurde erstmals eine Schallspitze mit einem Arousal verknüpft. Die Geräte waren in Echtzeit gekoppelt. Somit war es Freedman et al. möglich, akustische Arousals von nicht-akustischen zu unterscheiden (siehe Abbildung 1.2 S.13, aus Freedman et al. 2001 (S.452)).

Eine erhöhte Arousalanzahl kann Schlafdeprivation verursachen. Bei Schlafdeprivation kann sich auch die Arousalschwelle des Schläfers verändern. In der Studie über die Ad- 
Abbildung 1.2: Schall und Arousal

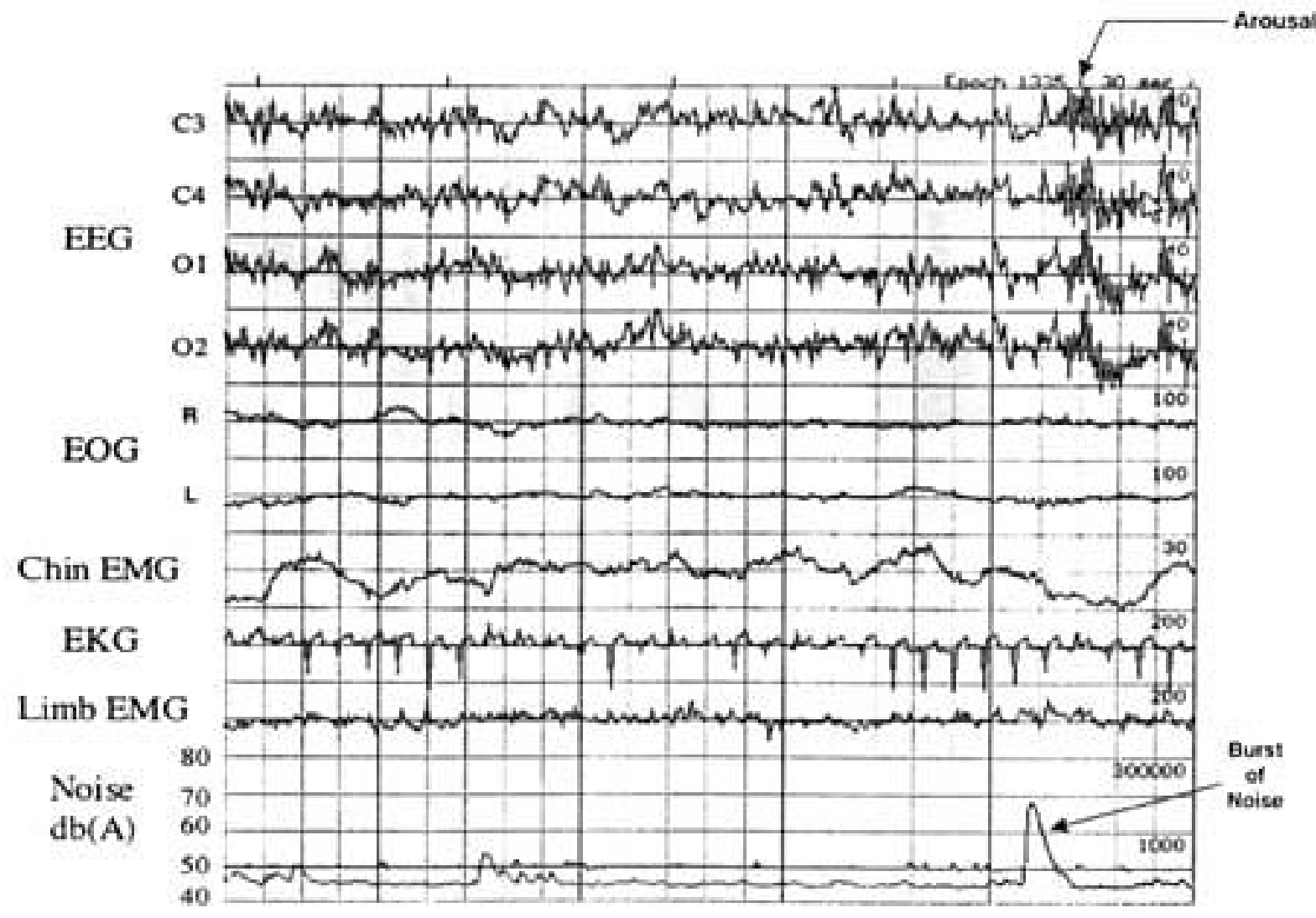

Polysomnographie mit 4 Kanälen (C3, C4, O1 und O2) der Elektroenzephalographie (EEG), rechtem und linkem Elektrookulogramm (EOG), Kinn- und Extremitätenelektromyogramm (EMG), EKG und fortwährender Schallpegelmessung (Mittlerer Schall). Das EEG ist repräsentativ für Schlafstadium S1 mit einem Arousal durch einen Umgebungslärm von $69 \mathrm{~dB}(\mathrm{~A})$ hervorgerufen.

Auf eine Schallspitze des Schallpegels (Pfeil: Burst of noise) folgt ein Arousal (Pfeil: Arousal) (Freedman et al. 2001 S.452). 
aptation der akustischen Arousalschwelle (AAT) bei $\delta$-Schlafentzug mussten Ferrara et al. 1999 feststellen, dass die Schwelle signifikant anstieg. Sie untersuchten Patienten, die kontrolliert bei Eintreten des $\delta$-Schlafs geweckt wurden. Somit verminderte sich der prozentuale Anteil an $\delta$ - und REM-Schlaf auf ein Minimum. Die Schlafstadien S1 und S2 waren vorherrschend. Daraufhin stellten sie fest, dass diese Patienten wesentlich schwerer auch im Stadium S1 oder S2 durch akustische Reize zu erwecken waren, als es bei Schlafgesunden in gleichem Schlafstadium sonst der Fall war. Bei vermindertem $\delta$-Schlaf, aber vermehrtem Schlafstadium S1 und S2 ist ein stärkerer akustischer Reiz notwendig, um den Schlafenden zu erwecken. Jedoch, so Ferrara et al., ist trotz der großen Adaptationsfähigkeit des menschlichen Körpers mit einer Minderung der Schlafqualität zu rechnen (Ferrara et al. 1999). Wie dargelegt wurde, ist auf den meisten Intensivstationen Lärm eine wesentliche Ursache für Störungen von Qualität und Quantität des Schlafes. Oft resultieren Schlafdeprivation und -fragmentation, die wiederum die Wahrnehmungsmöglichkeit und den mentalen Status eines Patienten beeinflussen. So kann der von Hansell im Jahre 1984 beschriebene Teufelskreis, Lärm Schlafdeprivation - Durchgangssyndrom, entstehen.

\subsubsection{Der Schlaf und die Psyche: Das Durchgangssyndrom}

Das Durchgangssyndrom ist laut McGuire et al. (2000) gekennzeichnet durch Antriebsstörungen, Gefühlsarmut, Verlangsamung aller psychischen Funktionen, Gedächtnisstörungen, schwankenden Bewusstseinsstand, schlechte Orientierung zu Ort, Zeit und Person, Wahnvorstellungen und Halluzinationen, Aggressivität oder Passivität (McGuire et al. 2000). Synonyme: ICU-Syndrom, ICU-Psychose, postoperatives Delirium, Delirium, Durchgangssyndrom

Ein Durchgangssyndrom kann früh auftreten und dauert oft ca. 24 - 48 Stunden, kann aber auch bis zu 14 Tagen andauern. Einige Autoren behaupten, dass die Inzidenz des Durchgangssyndroms auf einer ICU bei 36 Prozent der Patienten läge (McGuire et al. 2000, Dyer et al. 1995). Das bedeutet also, dass mehr als jeder dritte Patient 
auf der von McGuire et al. untersuchten Intensivstation an einem Durchgangssyndrom litt. Jedoch muss die Inzidenz des Durchgangssyndroms von der Art der Intensivstation abhängig gemacht werden. $\mathrm{Zu}$ den vielen pathophysiologischen Faktoren, die zu einem Durchgangssyndrom führen können, gehören metabolische Dysbalancen, Elektrolytentgleisungen, Infektionen, Schädelhirntraumen, Gefäßschäden und Minderperfusionen, zerebrale Schädigungen durch Hypoxämie oder Medikamente (Anästhetika, An-

algetika, Antibiotika, Anticholinergika, Antiarrhythmika oder Antihypertensiva usw.). Auch der prämorbide kognitive Status eines Patienten ist nach McGuire et al. entscheidend für die Ausprägung einer ICU-Psychose. Letztlich kommen noch psychologische Stressfaktoren, Schlafdeprivation, Lärm und andere Einflüsse der Umgebung hinzu. Jedoch konnten McGuire et al. 2000 keinen direkten Zusammenhang zwischen Schlafdeprivation, psychosozialem Stress, der Umgebung und dem Durchgangssyndrom nachweisen. Sie halten daher die postoperative Medikation für wesentlich.

Im Widerspruch dazu stellte Schwab (1994) fest, dass sich bei gesunden Probanden, die einem 2- bis 5-tägigen Schlafentzug ausgesetzt wurden, die Symptome eines Durchgangssyndroms zeigten (Schwab 1994). Schwab nannte Schlafdeprivation eine Hauptursache für ein Durchgangssyndrom. Zur Behandlung schlug er vor, die Reizüberflutung zu vermindern und Kommunikation sowie Orientierung der Patienten zu Ort und Zeit zu fördern. Aaron et al. (1996), Hansell (1984) und Schwab (1994) postulieren, dass Lärm, Arousals und Schlaffragmentierung das Durchgangssyndrom auslösen können. Auch Krachman et al. (1995) sowie McGuire et al. (2000) vermuteten ferner, dass damit ein verlängerter Krankenhausaufenthalt bzw. eine steigende Morbiditäts- und Mortalitätsrate assoziiert sein könnte.

\subsubsection{Schlaf und Heilung auf ICU}

Schlaf ist schon seit jeher mit Heilung in Verbindung gebracht worden. Eine Krankheit, welcher Genese auch immer, ist stets eine Herausforderung für Psyche, Geist und Körper. Aufrechterhaltung der Hämostase und Immunkompetenz stellen hohe Anfor- 
derungen an den Organismus. Die als Folge einer Operation oder Trauma auftretende typische Stoffwechselveränderung nennt man Postaggressionsstoffwechsel. Er ist durch eine katabole Stoffwechsellage und eine gestörte Substratverwertung gekennzeichnet. Der Energiebedarf ist gestiegen. Erholung und Ruhe sind in dieser Phase für den Patienten sehr wichtig. Zusätzlicher Stress für Geist und Körper, zum Beispiel in Form von Schlafentzug, psychosoziale Belastung, Lärm und fehlende Ansprache können die Heilung beeinträchtigen.

Carola et al. stellten 1990 die Hypothese auf, dass die NREM-Stadien S3 und S4 den größten Erholungswert für den Patienten haben. Wie schon erwähnt, ist die Schlafarchitektur des Patienten auf der Intensivstation dahingehend verändert, dass der $\delta$-Schlaf zugunsten der Schlafstadien S1 und S2 vermindert ist. Ein Schlaf mit verminderter $\delta$-Aktivität ist also weniger erholsam als der eines Gesunden. Carola et al. folgerten weiter, dass Schlafentzug eine zusätzliche Belastung für die Gesundheit des Patienten sei (Carola et al. 1990).

Auch Rosenberg et al. (1995) vertreten die Ansicht, dass das Umfeld einer ICU die Genesung eines Patienten ungünstig beeinflusst.

Um eine effektive Verbesserung der Heilungschancen zu erreichen, und nicht zuletzt um Dauer und Kosten eines Krankenhausaufenthaltes zu verringern, ist es notwendig, die Störungen und negativen Einflüsse auf den Heilungsverlauf zu kennen. Man sollte die Auswirkungen auf den Organismus einschätzen können, um Beeinträchtigungen durch eine intensivmedizinische Behandlung zu vermeiden. Sinnvoll ist es in diesem Zusammenhang deshalb, diejenigen Störungen der Patienten zu untersuchen, die auch beeinflusst werden können. Solche sind z.B. Störungen des Schlafverlaufes durch die Intensivumgebung. Daher sollen in dieser Untersuchung, auf Grundlage von bisher erhobenen Forschungsergebnissen, die folgenden Aufgaben und Fragen beantwortet werden. 


\subsection{Zielsetzung \& Fragestellung}

- Kritisch kranke Patienten einer Intensivstation des Uniklinikums Göttingen sollen von 22:00 bis 6:00 Uhr, 8 Stunden zeitgleich mit PSG und Schallpegelmessung überwacht werden.

- Es sollen Hypnogramme und Lärmprofile erstellt werden, um den Schlaf der Patienten besonders auf externe Einflüsse und eventuelle Schlafstörungen hin zu untersuchen. Dabei soll insbesondere der Einfluss des Lärms untersucht und dessen Ursache genauer bestimmt werden.

- Die Hypothese, dass ein erhöhter Schallpegel auf einer Intensivstation zu vermehrten Arousals und damit zu einer Reduzierung der Schlafquantität und -qualität führt, soll geprüft werden.

- Es sollen Lärmspitzen direkt mit dem Auftreten von Arousals verglichen werden, wie es erstmals Freedman et al. (2001), siehe auch Kapitel 1.2.1 (S. 10) beschrieben hatten. Jedoch sollen zusätzlich bestimmte akustische Arousals verifiziert werden, die einen Rückschluss darauf zulassen sollen, welche Lärmursachen häufig sind und gehäuft Arousals auslösen.

- Hierbei sollen unterschiedliche Arten der Arousals eruiert werden, die Rückschlüsse auf Arbeitsabläufe einer Intensivstation zulassen, um die Abläufe an die Bedürfnissen eines schwer Kranken anpassen zu können.

- Die erhobenen Schlafparameter sollen mit den Ergebnissen von Steinberg et al. (2000) und Freedman et al. (2001) verglichen werden.

- Weiter soll ein Zeitprofil erstellt werden, das das zeitliche Auftreten der verschiedenen Arousals illustriert.

- Letztlich sollen die erhobenen Schlafparameter auf Korrelation mit den erhobenen Arousalarten (ICU; nicht-ICU) überprüft werden. 


\section{Kapitel 2}

\section{Methoden}

\subsection{Studienart}

Es handelt sich um eine neurophysiologische Querschnittsuntersuchung an intensivmedizinisch hospitalisierten Patienten.

\subsection{Studienort}

Die Untersuchung ist auf den anästhesiologischen Intensivstationen 0112 und 0113 der Universitätsklinik Göttingen, Robert-Koch-Str. 40, 37075 Göttingen durchgeführt worden. Auf diesen Intensivstationen, mit 14 bzw. 8 Bettplätzen, sind postoperative Patienten und solche, die intensivpflichtiger Überwachung oder Behandlung bedürfen.

Einen Grundriss der Station 0112 zeigt Abbildung 2.1 (S.19).

Die Stationen sind in mehrere Zimmer unterteilt und bieten teilweise einen Sicht- und Lärmschutz der Patienten untereinander sowie zum Personal. Die Zimmer sind mit ein, zwei, drei oder vier Bettplätzen ausgestattet. Die Ein- und Zweibettzimmer befinden sich am weitesten entfernt von den Schwesternzentralen und sind von drei Seiten eingeschlossen und mit einer Schiebetür verschließbar; diese sind meist den weniger überwachungsbedürftigen Patienten vorbehalten. Die Vierbettzimmer befinden sich in unmittelbarer Nachbarschaft zur Schwesternzentrale und beherbergen hauptsächlich Patienten mit hohem Pflege- und Überwachungsniveau. Eine Abtrennung der Viererzimmer zum Pflegebereich ist nicht vorhanden. 
Abbildung 2.1: Lageplan der Intensivstation der Universität Göttingen 0112

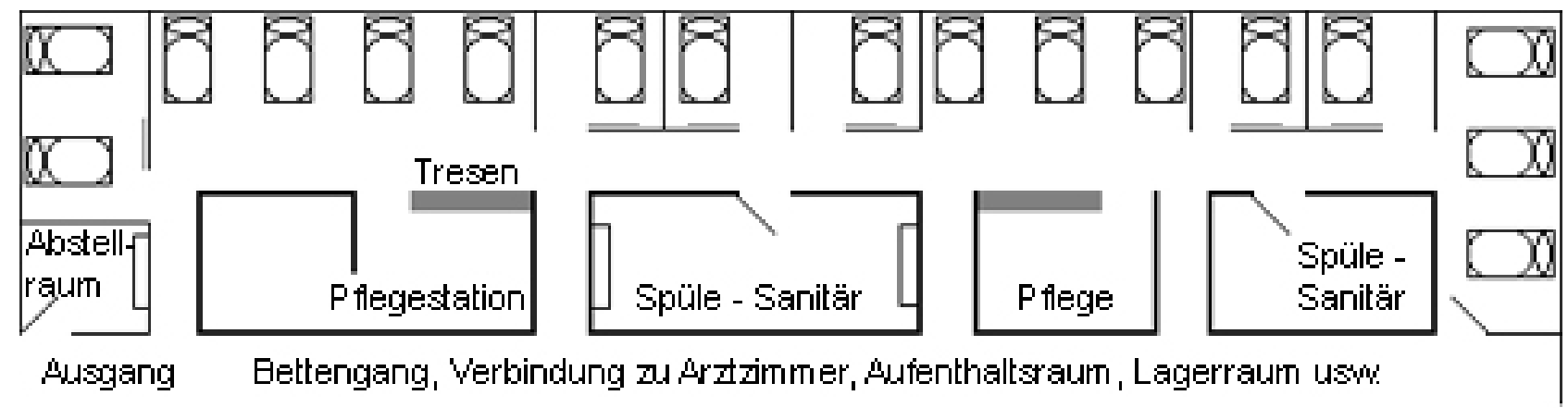

Die Stationen 0112 und 0113 sind ungefähr baugleich, sie sind spiegelverkehrt und unterscheiden sich in nur wenigen unwesentlichen Details.

\subsection{Studienpopulation}

Es wurden 31 Patienten im Zeitraum von Dezember 2000 bis September 2001 untersucht. Das mittlere Alter betrug $53 \pm 14,98$ Jahre (20 bis 76 Jahre), davon 21 Männer und 10 Frauen, die ein oder zwei Nächte je 8 Stunden mittels PSG überwacht wurden. Elf davon wurden in zwei aufeinanderfolgenden Nächten untersucht, die anderen 20 nur eine Nacht.

\subsubsection{Auswahl der Patienten}

Die Patienten wurden unter Berücksichtigung der Ein- und Ausschlusskriterien durch die ärztlichen Untersucher ausgewählt und nach eingehender Befragung und körperlicher Untersuchung in die Studie eingeschlossen. Die Patienten wurden ausführlich über den Inhalt der Studie unterrichtet, über Risikofaktoren aufgeklärt und gaben ihr schriftliches Einverständnis.

\subsubsection{Aufnahmekriterien}

In die Studie aufgenommen wurden Patienten im Alter zwischen 18 und 80 Jahren, die sich aus verschiedenen medizinischen Indikationen einer intensivmedizinischen Behandlung unterziehen mussten. Die Patienten mussten extubiert sein und spontan atmen. 


\subsubsection{Ausschlusskriterien}

- Manifeste cerebrale und/oder neuronale Erkrankung oder Schädigung.

- Bekannte vorbestehende Insomnie oder schlafgebundene Erkrankung.

- Bekannter vorbestehender Hypnotika- oder anderer Drogenabusus (insbesondere Alkohol).

- Bekannte vorbestehende Antidepressivamedikation.

- Beatmete Patienten.

- Erforderliche Akutintervention während der Untersuchung (Reanimation, notfallmäßige Intubation, etc.)

- Septische oder infektiöse Patienten.

\subsubsection{Abbruchkriterien}

Der Studienabbruch erfolgte bei Auftreten eines der Ausschlusskriterien, einer beeinträchtigenden unerwünschten Wirkung oder auf Wunsch des Patienten, wobei hierfür ausdrücklich keine Gründe genannt werden mussten. Vier Patienten brachen die Messung vorzeitig ab.

\subsubsection{Ethik und Sicherheit}

Die Untersuchung wurde entsprechend den Grundsätzen der revidierten Deklaration des Weltärztebundes von Helsinki in der aktuell gültigen Fassung und in der "Note for Guidance in Good Clinical Practice“ GCMP (GCMP/ICH/135/95, gültig ab 17.1.97) durchgeführt (CPMP 1990). Das Studienprotokoll wurde der medizinischen Ethikkommission der Georg-August-Universität vorgelegt (lfd. Antragsnummer 10/06/00) und am 01.01.01 von ihr genehmigt.

Die Vitalparameter und Schlafparameter wurden online erfasst. Gleichzeitig befanden sich die Patienten unter Sichtkontrolle des Untersuchers. Über den gesamten Untersuchungszeitraum stand die Studie unter ärztlicher Überwachung. 


\subsection{Studienablauf}

Um 21:00 Uhr begann die circa 60-minütige Vorbereitung der Messung. Die PSG wurde über 8 Stunden von 22:00 bis 6:00 Uhr aufgezeichnet. In der Vorbereitungszeit wurde der Patient an die Messgeräte PSG und Pulsoximeter des MEPALß)-Systems angeschlossen. Die Elektroden für die PSG-Ableitung wurden nach den internationalen Kriterien von Rechtschaffen und Kales (1968), den Richtlinien der Deutschen Gesellschaft für Schlafmedizin (DGSM) (Penzel et al. 1993) und dem internationalen 10/20 System (Jasper 1958) angebracht. Die externen Messgeräte (Schallpegelmessgerät, Pulsoximeter) wurden neben dem Kopfende des Patientenbettes positioniert und mit der PSG und dem Pulsoximeter am Computer angeschlossen. Mit Hilfe der Software DataLab ${ }^{\mathrm{TM}}$ von Medcare $^{\mathrm{TM}}$ (s. Kapitel 2.4.4 Seite 24) konnte die Impedanz der Elektroden gemessen und bei Bedarf die Ableitung korrigiert werden. Waren die Ableitungen qualitativ zufriedenstellend, konnte die Messung beginnen. Ein Untersucher befand sich für die Dauer der Messung in der Nähe des Patienten und notierte online alle Aktivitäten des Patienten sowie der Schwestern und Ärzte. Einschränkungen der Pflege oder Behandlung wurden seitens der Untersucher nicht vorgenommen. Das Pflegepersonal ist darauf hingewiesen worden, dass der Patient jede Behandlung oder Medikation bekommen solle, die vorgesehen war. Es wurde darauf geachtet, dass sich der Patient unter intensivmedizinischen Normalbedingungen befand. Die Messung wurde morgens um 6:00 Uhr beendet. Meist waren die Patienten zu diesem Zeitpunkt schon wach. Ist zu einem früheren Zeitpunkt schon abzusehen gewesen, dass der Patient nicht mehr schlafen würde, konnte die Messung auch eher beendet werden, nicht jedoch vor 5:30 Uhr. Falls der Patient um 6:00 Uhr noch schlief, wurde er geweckt.

\subsubsection{Untersuchungsverfahren und Aufbau}

Die Polysomnographie wurde mit dem mobilen Schlaflabor MEPALß)-System (MAP, Martinsried) durchgeführt. Das Schlaflabor befand sich auf einem fahrbaren Computertisch und bestand aus folgenden Komponenten: Polysomnographischer Ableitkopf, Pulsoximeter, PSGVerstärker, Computersystem (PC) mit der Schlaflabor-Software DataLab ${ }^{\mathrm{TM}}$ und der Auswertungssoftware Analysis Manager ${ }^{\mathrm{TM}}$ sowie den nichtinvasiven Elektroden und Sensoren; ferner wurde ein Schallpegelmessgerät, das 'Soundlevelmeter SL-4001' der Firma Lutron verwendet. 


\section{Abbildung 2.2: Die Platzierung der Elektroden nach dem internationalen} 10/20-System (Jasper 1958)
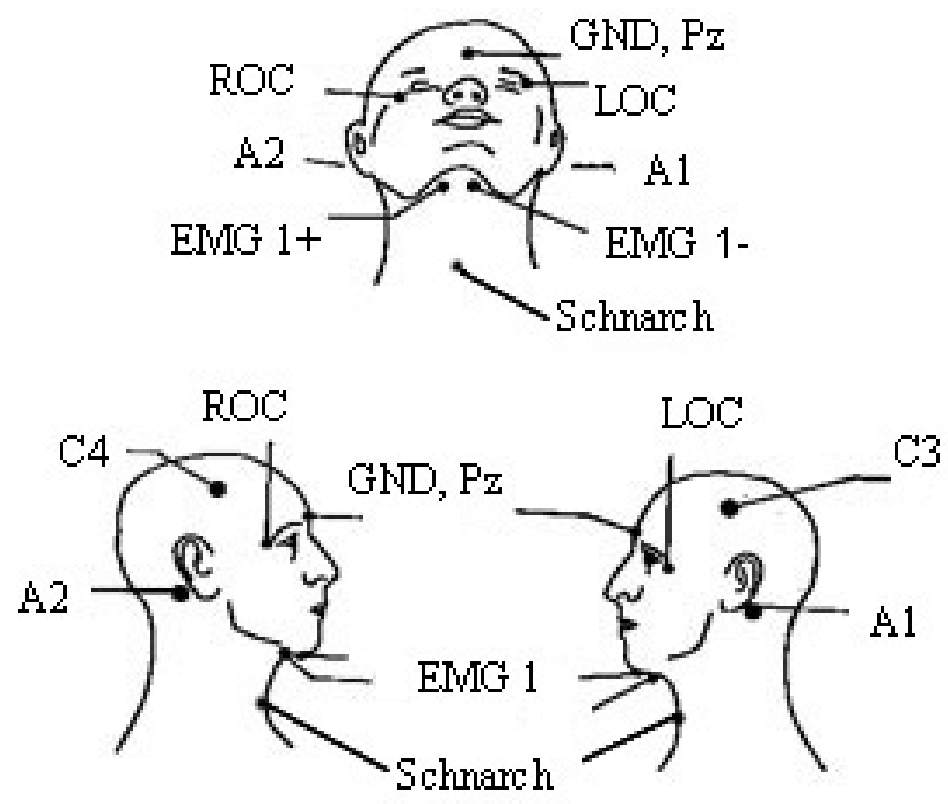

Es befand sich ein Mikrophon 'Schnarch' auf Höhe des Kehlkopfes. Modifiziert nach Rechtschaffen und Kales 1968 (S. 19)

\subsubsection{Polysomnographie}

Für die Polysomnographie wurde das 32-Kanal-System MEPAL@von MAP benutzt. Die abgeleiteten und im Folgenden aufgelisteten Messparameter dienten sowohl zum Erkennen der Schlafstadien, als auch der Erstellung eines Schallprofils. Die Kanäle EEG1, EEG2, EOG1, EOG2 und EMG1 (Kinn) dienten der Bestimmung der Schlafstadien, aus denen dann ein Schlafprofil, ein sogenanntes Hypnogramm, erstellt werden konnte. Die EEG und EOG Elektroden wurden an den Stellen C3, C4, A1, A2, GND und Pz sowie LOC und ROC nach dem internationalen 10/20 System platziert. (Abb. 2.2 S.22). Die Goldelektroden C3 und C4 wurden mit zehn-prozentigem Collodium befestigt. Mit Kontaktgel wurde eine qualitativ hochwertige Ableitung erreicht. Die übrigen Elektroden waren selbstklebend für die 
EEG-Ableitung geeignet. Die beiden EMG-Elektroden am Kinn wurden zwischen den Mandibularbögen leicht versetzt angebracht. EMG2 (Bein links), EMG3 (Bein rechts) und ein Mikrophon, im Weiteren als Kanal 'Schnarch' benannt, unterstützten die Auswertung zur Diagnose von Schlafstadien oder Arousals. Zwei Beinelektroden wurden jeweils auf dem Musculus tibialis anterior proximal im Abstand von ca. $10 \mathrm{~cm}$ angebracht.

Die Atmung kann durch den Atemflussgeschwindigkeitsmesser, den sogenannten 'Flow', den Thoraxsensor, den Abdomensensor, sowie die Sauerstoffsättigung des Blutes $\left(\mathrm{SaO}_{2}\right)$ und das Kehlkopfmikrophon (Kanal 'Schnarch') hinreichend beurteilt werden. Die Thorax- und Abdomensensoren bestanden aus Gurten, die auf der jeweiligen Höhe um den Patienten gelegt wurden. Sensoren konnten über Dehnung die Atemexkursion des Patienten feststellen.

Mit Elektroden wurden EKG und Herzfrequenz (HF) bestimmt. Die EKG-Elektroden wurden im zweiten rechten bzw. im fünften linken Interkostalraum parasternal befestigt. Die Herzfrequenz und die Sättigung konnten über das Pulsoximeter, dessen Sensor an einem Finger befestigt wurde, berechnet und in den Rechner eingespeist werden.

Die genannten Elektroden, außer dem Pulsoximeter, wurden an dem sogenannten Ableitkopf zusammengeführt, dem ein Verstärker nachgeschaltet war. Hiernach folgte die Verbindung zum PC, in dem die Daten aufgenommen wurden (Siehe Abbildung 2.3 S.25). Die polysomnographische Aufzeichnung wurde während der Messung permanent vom Untersucher kontrolliert. Wurden Auffälligkeiten in den Ableitungen festgestellt, die auf eine Verschlechterung des Gesundheitszustandes zurückzuführen waren, sollte der Versuch abgebrochen werden. Dieser Fall trat bei keinem der Patienten ein.

Die abgeleiteten und registrierten Parameter werden im Anhang Tabelle 6.2 (S. 86) aufgelistet.

\subsubsection{Externe Geräte: Schallpegelmessgerät, Pulsoximeter}

Die Schallpegelmessung wurde mit dem Gerät 'Lutron SL-4001 Soundlevelmeter' durchgeführt. Es wurde in einem Bereich von 20-100 dB gemessen. Das Schallpegelmessgerät ist neben den Patienten am Kopfende in einem Meter Entfernung platziert worden. Der Schallpegel wurde über den Adapter in den Computer eingespeist. Somit ist jedem Zeitpunkt ein Schallwert zuzuordnen. Gemessen wurde in Dezibel. 
Bei Ereignissen, die den Schläfer erwecken oder stören konnten, oder akustischen Reizen wurden entsprechende Eintragungen zeitgleich in die Ereignisdatei der DataLab ${ }^{\mathrm{TM}}$ Software vorgenommen. Der externe Adapter machte es möglich, die Daten externer Geräte zeitgleich aufzunehmen. An den externen Adapter wurde auch das 'Pulse Oximeter Model 8600' der Firma 'Nonin Medical Inc.' angeschlossen.

\subsubsection{Computergestützte Registrierung der Daten}

Die Daten von Schallpegel und Pulsoximeter wurden über einen externen Adapter, die PSG mit Hilfe des Ableitkopfes (30-Channel-Sleep-Jackbox) und eines anschließenden PSG-Verstärkers mit AD-Wandler (30-Channel-Sleep-Patient-Amplifier) über eine Schnittstelle in den Computer eingespeist. Der Computer und Monitor waren durch einen Trenntransformator vor Netzstörungen geschützt (siehe Abbildung 2.3 S.25).

Die verwendete Software DataLab ${ }^{\mathrm{TM}}$ diente zur Aufnahme der Daten und AnalysisMana-

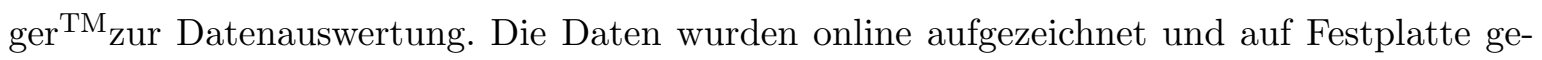
speichert, um zu einem späteren Zeitpunkt ausgewertet zu werden. Der Untersucher konnte mit der Software DataLab ${ }^{\mathrm{TM}_{\text {zeitgleich }}}$ in die digitale Aufzeichnung Ereignisse in einer Ereignisliste dokumentieren. Diese Ereignisse konnten bei der Auswertung einer oder mehreren Epochen zugeordnet werden, und somit zum Beispiel einem Arousal zugewiesen werden. Bei den Ereignissen handelte es sich meist um Gerätealarme, Geräusche von Schwestern, Ärzten oder Patienten. Auch Manipulationen am Patienten oder Aktionen des Patienten selbst konnten Auslöser eines akustischen Ereignisses sein (s.a. Kap. 2.8 S.35). Der Untersucher überprüfte auch die Aufnahmequalität. Verschlechterte sich die Qualität eines Signals, wurde die Elektrode ausgetauscht. Der Patient konnte dabei erweckt werden. Auch diese Tätigkeiten am Patienten wurden in der Ereignisliste festgehalten.

\subsection{Untersuchungsdurchführung}

Vor Aufnahme der Messdaten wurden die Patienten nach dem Ramsay-Score, dem SAPS II und dem EPS-Score eingeteilt. Der Ramsay-Score (Siehe Tab. 2.1) ist ein etabliertes Verfah- 
Abbildung 2.3: Versuchsaufbau

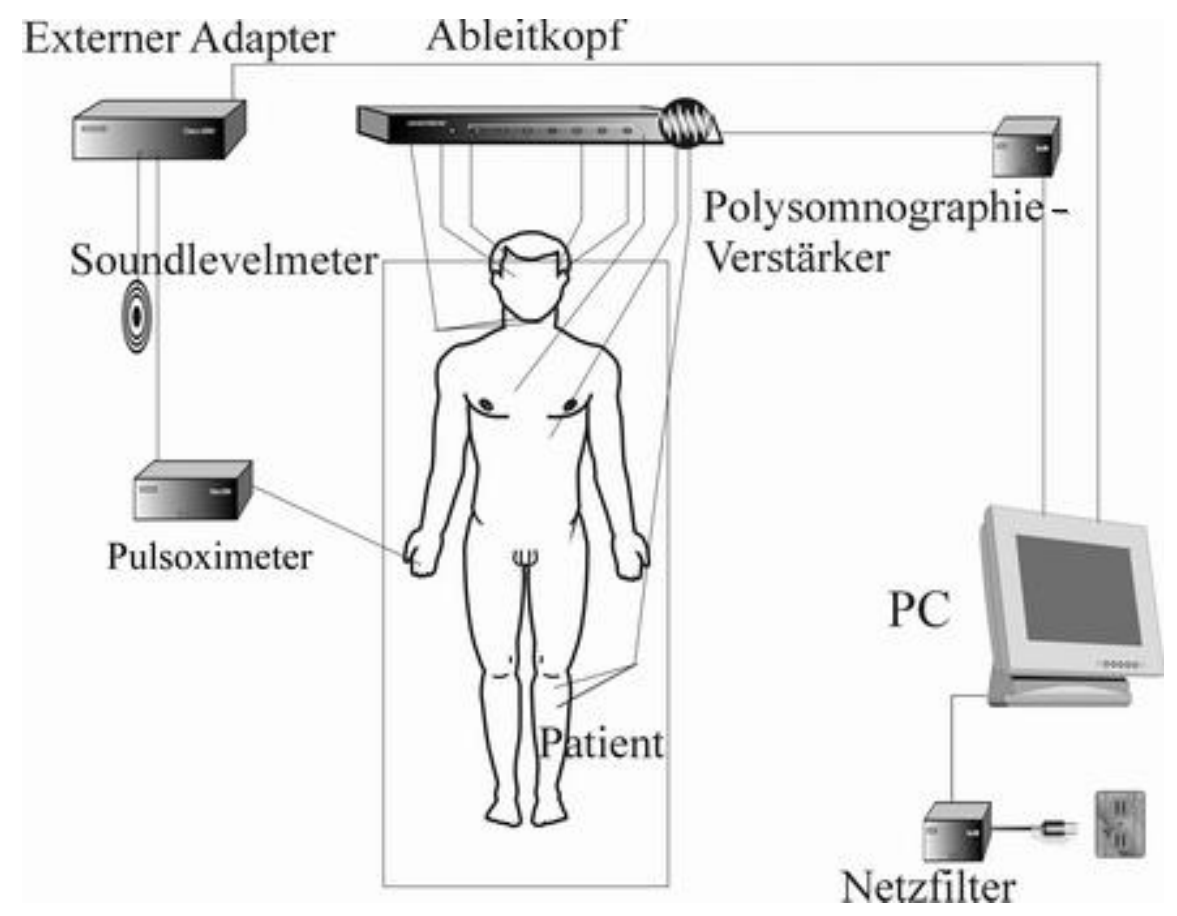

Aufbauschema der eingesetzten Untersuchungsgeräte. 
Tabelle 2.1: Ramsay-Score: die 6 Grade der Sedierung eines Patienten (Ramsay et al. 1974)

- Wach

1. Patient ängstlich und agil oder unruhig oder beides

2. Patient kooperativ orientiert, und ruhig

3. Patient reagiert nur auf Kommando

- Schlaf: Der Patient reagiert auf einen leichten Schlag auf die Glabella oder auf ein lautes akustisches Signal mit

4. lebhafter Antwort

5. träger Antwort

6. keiner Antwort

ren, den Sedierungsgrad eines Patienten einzuordnen (De Jonghe et al. 2000 und Ramsay et al. 1974).

Um die Umgebung des Patienten besser einzuschätzen, wurde die Zahl der Betten im Zimmer notiert und die darin befindlichen Nachbarpatienten ebenfalls nach einem eigens entwickeltem Score eingeteilt, der aus drei Kategorien bestand: der Vigilanz (Erregungszustand) und der Pflegeintensität des Patienten, sowie dem zu erwartenden Schallpegel des oder der Nachbarpatienten. Diese Einteilung wird im folgenden EPS-Score genannt (Siehe Tab. 2.2).

Diese Einteilung erlaubte es, die Vigilanz der Patienten, den Grad der Pflegeintensität und den Grad des zu erwartenden Schallpegels abzulesen. Zu Beginn wurde dem Schläfer und falls vorhanden - den Nachbarpatienten ein Score zugewiesen. Befanden sich ein oder mehr Nachbarpatienten im Raum, so wurde der Score des Patienten mit dem höchsten EPS-Score übernommen und war somit stellvertretend für die Umgebung des untersuchten Patienten. Letztlich wurde der SAPS II Score ermittelt, der das Outcome der Patienten und die Mortalität erfasst. Le Gall et al. (1993) ermittelten den Simplified acute Physiology Score (SAPS 


\section{Tabelle 2.2: Die Kategorien des EPS-Score:}

1 Patient ruhig; wenig Pflegeintensität; kaum Interventionen geringer zu erwartender Schallpegel

2 Patient ruhig; pflegeintensiv; mäßige aber regelmäßige Interventionen; mittlerer zu erwartender Schallpegel

3 Patient unruhig; sehr pflegeintensiv; ständige Interventionen; großer zu erwartender Schallpegel

II), der aus 12 physiologischen Variablen besteht. Darunter werden das Alter, der Grund der Einweisung (elektiver oder notfallmäßiger chirurgischer Eingriff oder andere medizinische Gründe), und drei zugrunde liegende Krankheiten (AIDS, metastasierter Tumor oder hämatologische Erkrankungen) zusammengefasst. Ein erhöhter Score bedeutet eine schlechte Prognose, ein niedriger spricht für eine gute Prognose (Le Gall et al. 1993).

Um die Qualität der Ableitung zu überprüfen, wurde eine Impedanzkontrolle durchgeführt. Dazu wurde ein Kalibriersignal über alle EEG-Kanäle des Verstärkers für die aktiven und die Referenzelektroden erzeugt. Dabei wurden die Impedanzwiderstände gemessen, wobei Widerstände bis zu $10 \mathrm{k} \Omega$ zugelassen wurden. Das Maximum der EEG- und EOG-Widerstände lag bei $5 \mathrm{k} \Omega$. Hohe Impedanzwiderstände beeinträchtigen die Qualität einer hochwertigen Ableitung. Ein in die Software integrierter Netzfilter unterdrückte die 50-Hz-Frequenzen. Daraufhin folgte die physiologische Kalibrierung in Form einer Bioeichung. Man ließ den Patienten definierte Tätigkeiten durchführen, die in der Ereignisliste dokumentiert wurden. Tätigkeiten wie zum Beispiel Augenblinzeln, Augenrollen, Sprechen oder Husten konnten aufgrund des charakteristischen EEG-Musters erkannt und für die Schlafstadienanalyse benutzt werden (Siehe Kap. 2.6 S.28).

Nach Beendigung der Vorbereitungen und mit dem Signal "Licht löschen" wurde die Messung begonnen. Dies wurde mit den Worten "Lights Off" in die Ereignisliste eingetragen. Ab diesem Zeitpunkt konnte der Patient ungestört schlafen. Alle Ereignisse wurden dokumentiert (Beispiele der Ereignisse siehe Liste 6.1 S.87 im Anhang.) Das Ereignis "Lights On" setzte man, wenn das Licht Morgens eingeschaltet und die Messung beendet wurde. 
Die Elektroden und Sensoren wurden vom Patienten gelöst. Die Schlafmarkierungen "Lights Off" und "Lights On" bezeichneten jeweils den Beginn und das Ende der Messung. In einer Nacht entstanden dabei ca. 80 MB große Dateien, die auf einer Festplatte zur nachfolgenden Auswertung gespeichert wurden.

\subsection{Schlafstadienanalyse, Schlafparameter, Arousal}

Die aufgenommenen Daten aller Messungen wurden einer Schlafstadienanalyse inklusive Beurteilung des Schallpegels unterzogen, die Schlafparameter berechnet sowie die Arousals und Wachphasen bestimmt. Dann wurden die Daten auf Vollständigkeit, Validität und Aussagekraft überprüft.

\subsubsection{Auswertung und Schlafstadienanalyse der PSG}

Die Auswertung der Polysomnographie erfolgte nach den internationalen Kriterien von Rechtschaffen und Kales (1968) und den Richtlinien der DGSM (Penzel et al. 1993) am Computer mit der Software SleepView ${ }^{\mathrm{TM}}$. Jede Messung wurde in 30 Sekunden lange Epochen eingeteilt. Jeder Epoche wurde ein Schlafstadium S1, S2, S3, S4, REM, Wach (W) oder Movementtime (MT) zugeordnet. Die aufgenommenen Schlafstadien können graphisch in einem Hypnogramm dargestellt werden. Aus dem Hypnogramm ist die Schlafarchitektur des Patienten zu entnehmen. Es ist dadurch möglich, die Qualität des Schlafes abzuschätzen und mit anderen Hypnogrammen zu vergleichen (Siehe Abbildung 4.2 S.71).

\subsubsection{Lärm}

Der Lärmpegel wurde in Echtzeit mit Hilfe der PSG aufgenommen. Jedem Zeitpunkt wurde ein bestimmter Wert in Dezibel zugewiesen. Zusätzlich errechneten wir den Mittelwert, das Maximum, das Minimum und die Standardabweichung einer gesamten Messung und dann zu den einzelnen Zeitpunkten Eins bis Vier von 22:00 bis 0:00 Uhr; 0:00 bis 2:00 Uhr; 2:00 bis 4:00 Uhr und 4:00 bis 6:00 Uhr. 


\subsubsection{Die Schlafparameter}

Mit den gewonnenen Daten der Schlafstadienanalyse ließen sich Schlafparameter, wie Schlafeffizienz (SEI), "total sleep time“ (TST) und die Dauer der einzelnen Schlafstadien (W, NREM und REM) automatisch berechnen. In Tabelle 2.3 (S.30) sind die Schlafparameter aufgeführt, die benötigt wurden, um den Schlaf eines Patienten qualitativ und quantitativ zu beschreiben. Diesen Werten kam eine besondere Bedeutung zu da sie in dieser Untersuchung die Qualitätsmerkmale sein sollen. Tabelle 4.1 (S.66) erklärt die Berechnung und genaue Bedeutung der einzelnen Parameter.

\subsubsection{Arousals und Wachphasen}

Die Bestimmung der Arousals erfolgte computergestützt nach der aktuellen Arousalklassifikation der ASDA (1997), die auf der Definition von Rechtschaffen und Kales (1968) basiert

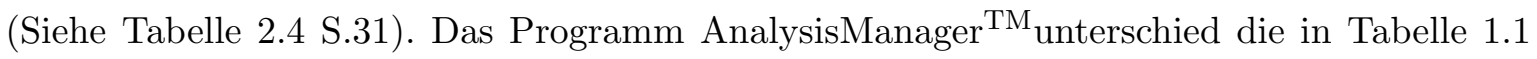
(S.8) aufgeführten Arousals. Die Auswertung wurde nachfolgend überprüft und gegebenenfalls korrigiert. Die ICU-Arousals mussten manuell nachgetragen werden (Siehe Kapitel 2.6.5 S.32). Rechtschaffen und Kales beschreiben das Arousal als eine Steigerung der Aktivität in jedem Kanal bei herrschendem Schlaf, die nur kurz, nicht aber über 15 Sekunden also die Hälfte einer Epoche andauert. Ein Arousal ist Ausdruck einer kurzen Bewegung wie zum Beispiel Entspannung der Gesichtsmuskulatur oder Blinzeln, kann einen Schlafstadienwechsel anzeigen und unterbricht die Kontinuität des herrschenden Schlafes in irgendeiner Weise (Rechtschaffen und Kales 1968 S.8). Ergänzend und aktuell findet man eine erweiterte Definition des Arousals von der American Sleep Disorders Association nach den internationalen Kriterien von Rechtschaffen und Kales 1968 und den Richtlinien der DGSM (ASDA 1997, Rechtschaffen und Kales 1968, Penzel et al. 1993).

Wir beschränkten uns auf die Standard-Arousals A:, A:LM, A:PLM, A:APN und A:HYPN sowie A.SNORE (Definition siehe Tabelle 1.1 S.8). Alle anderen Arousals wurden unter die Hauptgruppen zusammengefasst. Es wurden nur Hypopnoe- oder Apnoe-Arousal klassifiziert. Alle Ereignisse denen eine Extremitätenbewegung zugrunde lag, wurde als A:LM eingeteilt. Unterlagen mehrere Arousals einer Periodik wurden diese als A:PLM klassifiziert. Spontane Arousals waren weder den Standard-Arousals, noch der Klasse der ICU-Arousals zuzuordnen. 
Tabelle 2.3: Schlafparameter zur Qualifizierung und Quantifizierung des Schlafs nach Steinberg et al. (2000) (S.146)

\begin{tabular}{|c|c|c|}
\hline Abkürzung & Name & Bedeutung/Berechnung \\
\hline TIB & Time in Bed & $\begin{array}{l}\text { Zeit im Bett: Zeitraum von "Licht aus" } \\
\text { bis "Licht an" }\end{array}$ \\
\hline $\mathrm{SPT}$ & Sleep Period time & $\begin{array}{l}\text { Schlafperiodendauer: Zeit vom ersten } \\
\text { Stadium S1 bis zum Aufwachen }\end{array}$ \\
\hline TST & Total sleep time & Gesamte Schlafzeit ohne Wachphasen \\
\hline SEI & Sleep-Efficiency-Index & Schlafeffizienzindex TST/TIB $* 100$ \\
\hline SK & Schlafkontinuität & $\mathrm{TST} / \mathrm{SPT} * 100$ \\
\hline SOL1 & Sleep-Onset-Latency & $\begin{array}{l}\text { Schlaflatenz: Zeit von "Licht aus" bis } \\
\text { erstes Auftreten S1 }\end{array}$ \\
\hline S1 & Stadium 1 & \\
\hline $\mathrm{S} 2$ & Stadium 2 & \\
\hline $\mathrm{S} 3+\mathrm{S} 4$ & $\delta$-Schlaf $(\mathrm{S} 3+\mathrm{S} 4)$ & \\
\hline REM & Stadium REM & \\
\hline REM-Latenz & & $\begin{array}{l}\text { Zeit vom Lichtlöschen bis zum ersten } \\
\text { Auftreten REM }\end{array}$ \\
\hline Schall MW & Schall Mittelwert & $\begin{array}{l}\text { Durchschnittlicher Wert des Schallpe- } \\
\text { gels über SPT }\end{array}$ \\
\hline Standard-AI & $\begin{array}{l}\text { Index der Standard- } \\
\text { Arousals }\end{array}$ & $\begin{array}{l}\text { Durchschnittliche Anzahl aller } \\
\text { Standard-Arousals pro Stunde, be- } \\
\text { zogen auf TST }\end{array}$ \\
\hline ICU-AI & $\begin{array}{l}\text { Index der ICU- } \\
\text { Arousals }\end{array}$ & $\begin{array}{l}\text { Durchschnittliche Anzahl aller } \\
\text { Standard-Arousals pro Stunde, be- } \\
\text { zogen auf TST }\end{array}$ \\
\hline Gesamt-AI & $\begin{array}{l}\text { Index der Gesamt- } \\
\text { Arousals }\end{array}$ & $\begin{array}{l}\text { Durchschnittliche Anzahl aller Weckre- } \\
\text { aktionen pro Stunde, bezogen auf TST }\end{array}$ \\
\hline AWI & Index der Wachphasen & $\begin{array}{l}\text { Durchschnittliche Anzahl aller Wach- } \\
\text { phasen pro Stunde, bezogen auf SPT }\end{array}$ \\
\hline
\end{tabular}

Die Parameter wurden mit der Software DataLab ${ }^{\mathrm{TM}}$ errechnet. $^{2}$ 


\section{Tabelle 2.4: Die Arousal-Kriterien der ASDA zusammengefasst von Steinberg}

et al. (2000) nach den internationalen Kriterien von Rechtschaffen und Kales (1968) und den Richtlinien der DGSM (Penzel et al. 1993)

1. Für die Arousalklassifikation muss mindestens 10 Sekunden Schlaf vorausgehen. Arousals können auch im nach Rechtschaffen und Kales definierten Stadium 'Wach' auftreten, wenn dieses z.B. bis zu 14 Sekunden Schlaf enthält.

2. Zwischen zwei Arousals müssen mindestens 10 Sekunden Schlaf liegen.

3. Die Frequenzbeschleunigung im EEG muss mindestens für 3 Sekunden auftreten.

4. Arousals können im NREM Schlaf nur im EEG, ohne Anstieg im Muskeltonus des submentalen EMG auftreten. Im REM-Schlaf muss das Arousal von einer submentalen EMG-Erhöhung begleitet sein.

5. Ein alleiniger Anstieg im Muskeltonus reicht für die Arousalklassifikation nicht aus.

6. Artefakte, K-Komplexe oder $\delta$-Wellen werden nur dann als Arousals bewertet, wenn in mindestens einem Ableitkanal eine Frequenzbeschleunigung folgt.

7. 'Pen-blocking' Artefakte (Übersteuerung in einem Ableitkanal) werden dann als Arousals klassifiziert, wenn ihnen eine Frequenzbeschleunigung folgt.

8. Aufeinanderfolgende EEG- und EMG-Veränderungen mit einer jeweiligen Dauer kleiner 3 Sekunden, in der Summe aber über 3 Sekunden, werden nicht als Arousal klassifiziert.

9. Alpha Einstreuung in NREM mit einer Dauer kleiner 3 Sekunden und einer Frequenz größer 1 pro 10 Sekunden $(>0.1 \mathrm{~Hz})$ werden nicht als Arousal klassifiziert. Alpha-Einstreuungen mit einer Dauer größer 3 Sekunden werden nicht als Arousals klassifiziert, auch wenn in den vorausgehenden 10 Sekunden keine weitere Alpha-Einstreuung auftrat.

10. Schlafstadienwechsel stellen nicht das Kriterium eines Arousals dar.

(Steinberg et al. 2000 (S.148), ASDA 1997, Rechtschaffen und Kales 1968, Penzel et al. 1993) 


\subsubsection{Selbstdefinierte Arousals: "ICU-Arousals"}

Ziel dieser Untersuchung war es, die Einflüsse einer Intensivstation auf den Schlaf des Patienten zu erfassen. Da in einer normalen Schlafumgebung regelmäßige Störereignisse nicht die Regel sind, war es nötig, die Einflüsse genauer zu benennen. Als Störfaktoren wurden akustische, taktile und gemischt akustisch-taktile Einflüsse angesehen. Wir untersuchten dann ob ein dokumentiertes Ereignis mit einem Arousal verknüpft war oder nicht.

Die ICU-Arousalkriterien beinhalten die Arousalklassifikation nach den Kriterien von Rechtschaffen und Kales (1968) und den erweiterten Arousalklassifikationen der ASDA (1997) (Kapitel 2.6.4 S.29) sowie die folgenden ICU-Arousal-Kriterien.

Die akustische Lärmpegelschwelle von 10dB wurde dem Vorschlag Freedman et al. (2001) folgend gewählt (Siehe Abb. 1.2 S.13). Allerdings muss ein Lärmpegel von 10dB nicht zwingend eine Reaktion hervorrufen. Somit war gewährleistet, dass nicht jeder Minimalausschlag als Auslöser eines Arousals gezählt wurde. Es wurde eine Liste mit den charakteristischen Schallmustern der jeweiligen Geräte erstellt, die im Anhang (Abb. 6.1 S.84) einzusehen ist. Eine Sonderstellung nahm das undefinierte Lärm-Arousal ein. Bei diesem musste nur ein starker akustischer Reiz maximal 10 Sekunden einem Arousal vorausgegangen sein. Eine Dokumentation in der Ereignisliste war nicht nötig. Ein charakteristisches Schallbild musste nicht vorherrschen (Siehe Tabelle 2.6 S.34).

\subsection{Prüfung der Messungen}

Jede Messung wurde einer Qualitätsprüfung unterzogen, bevor sie in die Auswertung aufgenommen wurde.

1. Die Messung wurde auf Vollständigkeit geprüft.

2. Die Qualität der PSG musste eine zuverlässige Schlafstadienanalyse zulassen.

3. Die Ableitung des Schallkanals wurde auf Fehler und Vollständigkeit geprüft.

Zu 1 .

Zunächst wurde auf Vollständigkeit geprüft. Die Dauer der Messung musste mindestens über 6 Stunden im Zeitraum von 22:00 bis 6:00 Uhr durchgeführt werden, der Patient musste in 


\section{Tabelle 2.5: Die Kriterien der ICU-Arousals:}

\section{- Dem akustischen Arousal}

musste ein Ereignis maximal 10 Sekunden vorausgehen und in der Ereignisliste dokumentiert sein. das Ereignis muss in einer Anhebung der Schallkurve um mindestens 10dB gegenüber dem Minimalwert der Epoche im Schallkanal ersichtlich sein. Wurde das Ereignis nicht dokumentiert, konnte anhand der Beispielliste für charakteristische Schallkurven ein Ereignis zugeordnet werden. Die Arousals waren bedingt durch Monitor, Perfusor, Beatmungsgerät, und Telefon, sowie durch undefinierten Lärm.

- Das undefinierte Lärm-Arousal:

Das ' 'undefinierte Arousal"' war ein Geräuschereignis, das der genannten Erhebung des Schallpegels entsprach. War dieses Ereignis keinem charakteristischen Geräuschbild zuzuordnen, so wurde eine nachfolgende Weckreaktion ' 'undefiniertes Lärm-Arousal'" genannt.

- Dem taktilen Arousal musste ein Ereignis im Sinne einer Intervention wie Pflegen, Waschen, Blutabnahme o.ä. vorausgegangen und als solches in die Ereignisliste eingetragen worden sein. Es konnte maximal 10 Sekunden vor dem Arousal stattgefunden haben. Eine akustische Begleitaktion in Form einer Schallpegelerhöhung konnte vorkommen. Diese taktilen Weckreaktionen wurden 'Interventions-Arousals' genannt.

\section{- Dem gemischt akustisch-taktilen Arousal}

ging ein Ereignis maximal 10 Sekunden voraus. Das Ereignis konnte in der Ereignisliste festgehalten worden sein. Ein charakteristisches Geräuschbild im Geräuschkanal war notwendig. Als einziger und wichtigster Vertreter ist das Blutdruckmessgerät zu nennen, weil hierbei gleichzeitig sowohl ein akustischer als auch ein taktiler Reiz aufgenommen werden kann. Dieses wurde 'RR-Arousal' genannt.

\section{- Für alle ICU-Arousals gilt,}

dass kein Standard-Arousal oder ein anderes ICU-Arousal gleichzeitig bestehen kann. 


\section{Tabelle 2.6: Definition ICU-Arousal}

\begin{tabular}{|c|c|c|}
\hline ICU-Arousal & $\mathrm{a} / \mathrm{t}$ & Definition \\
\hline $\mathrm{Be}$ & $\mathrm{a}$ & $\begin{array}{l}\text { Jedes Arousal, das durch den Alarm des Beatmungsgerätes des Pa- } \\
\text { tienten oder eines Nachbarpatienten ausgelöst wird und die ICU- } \\
\text { Arousalkriterien erfüllt. }\end{array}$ \\
\hline Monitor & $\mathrm{a}$ & $\begin{array}{l}\text { Jedes Arousal, das durch den Alarm des Monitors des Patien- } \\
\text { ten oder eines Nachbarpatienten ausgelöst wird und die ICU- } \\
\text { Arousalkriterien erfüllt. }\end{array}$ \\
\hline Perfusor & $\mathrm{a}$ & $\begin{array}{l}\text { Jedes Arousal, das durch den Alarm eines Perfusors des Pati- } \\
\text { enten oder eines Nachbarpatienten ausgelöst wird und die ICU- } \\
\text { Arousalkriterien erfüllt. }\end{array}$ \\
\hline Telefon & $\mathrm{a}$ & $\begin{array}{l}\text { Jedes Arousal, das durch das Klingeln des Telefons ausgelöst wird } \\
\text { und die ICU-Arousalkriterien erfüllt. }\end{array}$ \\
\hline Lärm undefiniert & $\mathrm{a}$ & $\begin{array}{l}\text { Jedes Arousal, das durch einen undefinierten Schallpeak, der nicht } \\
\text { indentifiziert werden konnte und nicht in der Ereignisliste angege- } \\
\text { ben wurde, ausgelöst wird und die ICU-Arousalkriterien erfüllt. }\end{array}$ \\
\hline onal & $\mathrm{a}$ & $\begin{array}{l}\text { Jedes Arousal, das durch jeglichen Lärm, der von Personal (Ärzte, } \\
\text { Schwestern oder andere) ausgeht, ausgelöst wird und die ICU- } \\
\text { Arousalkriterien erfüllt. Es kann nicht zum Interventions-Arousal } \\
\text { hinzugezählt werden. }\end{array}$ \\
\hline Nachbarpatient & $\mathrm{a}$ & $\begin{array}{l}\text { Jedes Arousal, das durch jeglichen Lärm, der vom Nachbarpatien- } \\
\text { ten, ausgeht, ausgelöst wird und die ICU-Arousalkriterien erfüllt. }\end{array}$ \\
\hline $\mathrm{RR}$ & $a \& t$ & $\begin{array}{l}\text { Jedes Arousal, das durch die automatische Blutdruckmessung aus- } \\
\text { gelöst wird und die ICU-Arousalkriterien erfüllt. }\end{array}$ \\
\hline Intervention & $\mathrm{t}$ & $\begin{array}{l}\text { Jedes Arousal, dass durch jegliche Intervention oder Manipulation } \\
\text { am Patienten ausgelöst wird und die ICU-Arousalkriterien erfüllt. }\end{array}$ \\
\hline
\end{tabular}

Neu definierte ICU-Arousals ermöglichen es, die Ursache eines Arousals auf einer Intensivstation genauer zu eruieren. a - akustisch; t - taktil 
diesem Zeitraum die Möglichkeit haben zu schlafen.

Zu 2.

Die Qualitätsprüfung war notwendig, um eine zuverlässige Schlafstadienauswertung durchzuführen. Primär sollte auf Kanäle geachtet werden, ohne die eine Schlafstadienauswertung nicht möglich ist (C3, C4, EOG1, EOG2, EMG1 Kinn, EMG2 Bein links, EMG3 Bein rechts und Schallkanal). Mangelnde Qualität der Ableitung und eine pathologisches EEG könnten die Qualität der Messung negativ beeinflussen, so dass eine eindeutige Schlafstadienanalyse nicht möglich sein könnte. Die Messung wurde je nach Eindeutigkeit der Analyse zugelassen. Zu 3.

Um die ICU-Arousals beurteilen zu können, wurde die Ableitung des Lärmpegels geprüft. War der Schallkanal fehlerhaft oder in dem angegebenen Messzeitraum von 22:00 - 6:00 Uhr nicht vollständig, wurde die Messung als mangelhaft bewertet und nicht in die Auswertung aufgenommen.

Somit entstand eine Gruppe mit einer Anzahl ' $m$ ' Patienten und ' $n$ ' Messungen. Alle Messungen sind mit Großbuchstaben numeriert worden. Diejenigen Patienten, die zwei aufeinander folgende Nächte mit PSG überwacht worden waren, erhielten eine zusätzliche Numerierung 1-7 und die jeweilige Endung ' $a$ ' oder ' $b$ ' für die erste oder zweite Nacht. Somit ergibt sich z.B. für die zweite Nacht einer Messung 'S' eines Patienten, der zweimal gemessen wurde, die Nummer 'S5b'. Die Zahl '5'steht für die Numerierung aller Patienten, die zwei Nächte gemessen wurden. Da jede Nacht eine andere alphabetische Zuordnung hat, kann man an der Zahl (in diesem Fall '5) erkennen, ob es sich um denselben Patienten handelt. Die Messung mit der Nummer ' $\mathrm{C}$ ' eines Patienten, der nur eine Nacht beobachtet wurde, erhielt deswegen keine weiteren Kennzeichnung. Siehe Tabellen 3.3 S.46, 3.4 S.47 und 6.3 Anhang S.88.

\subsection{Auswertung}

Nach Prüfung aller Messungen konnten die Daten ausgewertet werden. 
Tabelle 2.7: "Normal human sleep" nach Carskadon und Dement 2000 (S.20)

1. Der Schlaf beginnt mit NREM.

2. NREM- und REM-Schlaf alternieren mit einer Periode von ungefähr 90 Minuten.

3. $\delta$-Schlaf tritt vermehrt in dem ersten Drittel der Nacht auf und geht mit dem Anfang des Schlafes einher.

4. REM-Schlaf ist in dem letzten Drittel vorherrschend und ist mit dem circadianen Rhythmus der Körpertemperatur verbunden.

5. Während der Schlafperiode ist ein Proband zu 5\% 'Wach',

6. zu $2-5 \%$ in Schlafstadium S1,

7. zu $45-55 \%$ in $\mathrm{S} 2$,

8. zu $3-8 \%$ in S3 und

9. zu $10-15 \%$ in Schlafstadium S4.

(Daraus ergibt sich für $\delta$-Schlaf (S3+S4) 13-23\% des SPT.)

10. Somit ergibt sich für den NREM-Schlaf ca. $75-80 \%$ und den

11. REM-Schlaf ca. 20-25\% innerhalb von vier bis sechs einzelnen Schlafzyklen

\subsubsection{Vergleich mit den Ergebnissen von Carskadon und Stein- berg (2000)}

Die Ergebnisse der Auswertung dieser Untersuchung wurden hier mit den Ergebnissen von Carskadon und Dement (2000) sowie Steinberg et al. (2000) verglichen. Die Daten werden tabellarisch gegenüber gestellt und in Kapitel 4.5.3 diskutiert (S. 68). Carskadon und Dement beschreiben 2000 in ihrer Untersuchung, "normal Human Sleep", den normalen Schlaf im idealen Fall. Diesem normalen Schlaf lagen die Messungen mit PSG eines jungen männlichen Probanden im Alter von 19 Jahren zugrunde. Carskadon und Dement (2000) konnten folgende Aussagen über den "normalen Schlaf" treffen, die in Tabelle 2.7 S.36 dargestellt sind. 


\section{Tabelle 2.8: Akustische und non-akustische Arousals}

1. Akustische Arousals

- A-Beatmung, A-Monitor, A-Perfusor, A-Telefon A-Personal, A-Nachbarpatient und A-Schall undefiniert

2. Non-akustische Arousals

- A-Intervention, A-RR sowie die Standard-Arousals.

\subsubsection{Auswertung der Arousals}

Die Ergebnisse der Arousals werden in Form des Arousalindex sowie prozentual angegeben, um einen direkten Vergleich der Messungen untereinander ziehen zu können. Die Angaben der Indices erfolgen in Arousal pro Stunde (Arousal/h). Die Arousals wurden einerseits in Standard- und ICU-Arousals und andererseits in akustische und non-akustische Arousals unterteilt (Siehe Tab. 2.8 S.37).

\subsubsection{Akustische und non-akustische Arousals}

Es wurde nun eine zweite Unterteilung aller Arousaluntergruppen getroffen, um die Untersuchung auch mit den Ergebnissen von Freedman et al. (2001) vergleichen zu können. Hierzu wurden die Arousals nicht wie vorher in ICU-Arousals und Standard-Arousals unterteilt, sondern in "Lärm-bedingte" und "nicht-durch-Lärm-bedingte" Arousals. Sie wurden analog zu Freedman 'akustische'- und 'non-akustische' Arousals genannt (Siehe Tabelle 2.8 S.37).

Die Summe der akustischen und non-akustischen-Arousals ergab die Anzahl der GesamtArousals. Auch hier wurde der Arousalindex für jede Messung und schließlich der prozentuale Anteil des akustischen Arousalindex am Gesamt-Arousalindex bestimmt. Die Werte werden in Kapitel 4.5.5 (S.73) mit denen von Freedman et al. (2001) verglichen. 


\subsubsection{Das Arousalprofil}

Die Dauer der Messung von 22:00 bis 6:00 Uhr wurde in vier gleichmäßige Zeitabschnitte à zwei Stunden von 22:00-0:00, 0:00-2:00, 2:00-4:00 und 4:00-6:00 Uhr unterteilt.

Automatisch konnte mit Hilfe der Software zu jedem Zeitpunkt die Anzahl der Arousals, TST und AWI berechnet werden. Der Index wurde durch die Formel

$$
\begin{gathered}
\text { Anzahl Arousal oder Wachphasen } \\
\text { TST }(\text { min }) \\
=A W I \text { oder Win }) \\
\text { Wachphasen - Index }
\end{gathered}
$$

angegeben. Zu jedem Zeitpunkt wurden Median sowie obere und untere Quartile, als auch Minimum und Maximum für den Gesamt-Arousalindex, der Standard- und ICU-Arousalindex, der Anteil des ICU-Arousalindex am Gesamt-Arousalindex, sowie TST und AWI errechnet. Die gewonnenen Werte wurden in Boxplots dargestellt. Die entstandenen Boxplotgrafiken zeigen den Median, die Verteilung innerhalb der Quartile sowie Minimum und Maximum.

So wurde für alle Messungen ein Arousalprofil erstellt. Die Berechnung, sowie die graphische Darstellung erfolgten mit Hilfe von Excel 2000 und SPSS 9.0 für Windows.

\subsubsection{Auswertung des Schallkanal}

Der Schallkanal zeigte den Mittelwert, das Minimum und Maximum, sowie die Standardabweichung zu jeder Epoche an. Darauf erfolgte die Berechnung der jeweiligen Mittelwerte einer Messung.

\subsubsection{Das Lärmprofil}

Die Daten des Lärmprofils wurden analog zu dem des Arousalprofils zu den Zeitpunkten eins bis vier bestimmt. Die errechneten Daten bestanden aus Schallmaximum (Schall MAX), Schallminimum (Schall MIN) und Schallmittelwert (Schall MW).

\subsubsection{Statistik}

Folgende Parameter wurden auf Beziehungen untereinander nach Pearson und Spearmans Rho untersucht: 
Anteil ICU-Arousals vs TST, Anteil ICU-Arousals vs SEI, Anteil ICU-Arousals vs Schall MW, Anteil ICU-Arousals vs Schall Max, Anteil ICU-Arousals vs Nachbarn, Anteil ICUArousals vs Alter, Anteil ICU-Arousals vs SAPSII-Score, Anteil ICU-Arousals vs EPS-Score, Anteil ICU-Arousals vs Ramsay-Score, AWI vs Schallmax, Nachbarn vs Schall MW.

Wir bestimmten für jeden Vergleich ein Korrelationsmaß mit SPSS 9.0 für Windows. Es wurde bivariat, einseitig jeweils die Korrelation nach Pearson und Spearman-Rho berechnet, die in Tabellen zusammengefasst worden sind. Signifikante Korrelationen wurden grafisch in Form einer Trendlinie dargestellt.

\subsubsection{Zusammenfassung der Methodik:}

- Patienten einer Intensivstation wurden mit PSG und Schallpegelmessung 8 Stunden von 22:00 bis 6:00 Uhr beobachtet.

- Die gewonnenen Daten wurden auf Qualität geprüft.

- Die Daten wurden einer Schlafstadienanalyse unterzogen, Schlafparameter berechnet und Arousals ausgezählt.

- Die gewonnene Auswertung wurde mit den Ergebnissen von Carskadon und Steinberg verglichen.

- Für jeden Patient wurde ein Arousal- und Lärmprofil erstellt.

- Korrelationen nach Pearson und Spearman wurden gesucht, und bei Signifikanz eine Grafik erstellt. 


\section{Kapitel 3}

\section{Ergebnisse}

\subsection{Die Population}

Es wurden 31 Patienten im Zeitraum von Dezember 2000 bis September 2001 untersucht. Das mittlere Alter der 21 Männer und 10 Frauen, die ein oder zwei Nächte lang je 8 Stunden mit PSG überwacht wurden, betrug $53 \pm 14,98$ Jahre (20 bis 76 Jahre). Siehe Abb. 3.1 (S.41). Elf Probanden wurden in zwei aufeinander folgenden Nächten untersucht, die Anderen nur eine Nacht. Die Studie umfasst somit 42 Untersuchungen, von denen 17 aufgrund der Qualitätsprüfung nicht aufgenommen werden konnten. Vier schieden wegen einer technisch unbrauchbaren Schallpegelmessung aus und 4 Messungen wurden auf Wunsch des Patienten frühzeitig abgebrochen. Sieben Messungen waren artefaktüberlagert oder wiesen ein pathologisches EEG auf, sodass eine hinreichende und aussagekräftige Auswertung nicht möglich war. Ein Softwareproblem führte zum Verlust zweier Datensätze. Einmal musste die Messung wegen der morgendlichen Körperpflege eines Patienten abgebrochen worden.

Nach der Qualitätsprüfung wurden 25 der Messungen in die Auswertung aufgenommen. Die Patienten, deren Messungen ausgewertet wurden, waren im Mittel 52 $\pm 15,90$ Jahre alt (20 bis 76 Jahre). Keiner der Patienten war jünger als 20 Jahre. Drei Männer und drei Frauen waren im Alter von 20 bis 39 Jahren; elf Männer und eine Frau im Alter von 40 bis 59, sechzig Jahre und älter waren vier Männer und drei Frauen. Die Dauer des Aufenthalts auf der Intensivstation betrug $3 \pm 2,04$ Tage (1-8). Insgesamt wurden bei 11 Patienten am ersten postoperativen Tag Messungen durchgeführt. Acht Patienten wurden am zweiten oder dritten 


\section{Abbildung 3.1: Altersverteilung aller gemessenen Patienten}

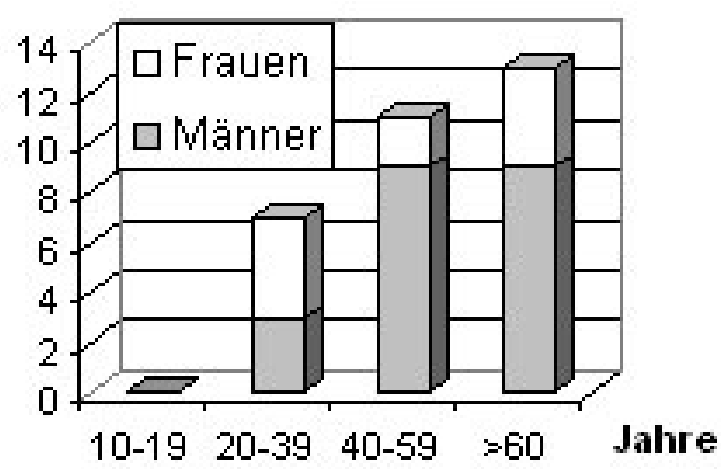

Kein Patient war jünger als 20 Jahre. Vier Patientinnen und 3 Patienten waren zwischen 20 und 39 Jahre alt, 9 respektive 2 zwischen 40 und 59. Älter als 60 Jahre waren 13, davon vier weiblich und 9 männlich. Der Altersdurchschnitt betrug $53 \pm 14,89$ Jahre (20 bis 76 Jahre).

postoperativen Tag gemessen, je drei am vierten oder fünften sowie am sechsten bis achten postoperativen Tag.

Sieben waren abdominalchirurgische und sechs traumatologische Patienten, drei orthopädische, je 2 HNO-ärztliche und je 2 kardiologische und thorakalchirurgische Patienten. Je ein Patient war gynäkologisch und urologisch. Ein weiterer Patient wurde internistisch geleitet. Alle Patienten erfuhren im Laufe ihres Aufenthaltes auf der Intensivstation eine Besserung ihres Gesundheitszustandes, sodass sie im Laufe der darauf folgenden Tage nicht mehr intensivpflichtig waren. Es konnten 6 Patienten in Einzelzimmern schlafen und 19 in Mehrbettzimmern. Sieben Patienten hatten einen Nachbarpatienten, sechs mussten das Zimmer mit 2 Nachbarn teilen und fünf Patienten hatten 3 Nachbarn.

Der gemittelte Ramsay-Score betrug 1,68 $\pm 0,69$, der gemittelte EPS-Score betrug 1,97 $\pm 0,43$. Der RAMSAY- und EPS-Score teilt sich auf wie Tabelle 3.1 (S. 42) zeigt. Ramsay unterschied in seiner Studie 6 Grade der Sedierung. Wobei die Einteilung 1-3 den wachen noch ansprechbaren Patienten und 4-6 den bereits schlafenden, sedierten Patienten beschreibt.

Drei Patienten konnten durch eine PCA-Pumpe die Schmerzmedikation selbst regulieren. Drei Patienten erhielten Diazepam, Remestan oder Dipidolor als Bedarfsmedikation. Alle biome- 
Abbildung 3.2: Überblick Nachbarpatienten

\section{Übersicht Ilachbarpatienten}

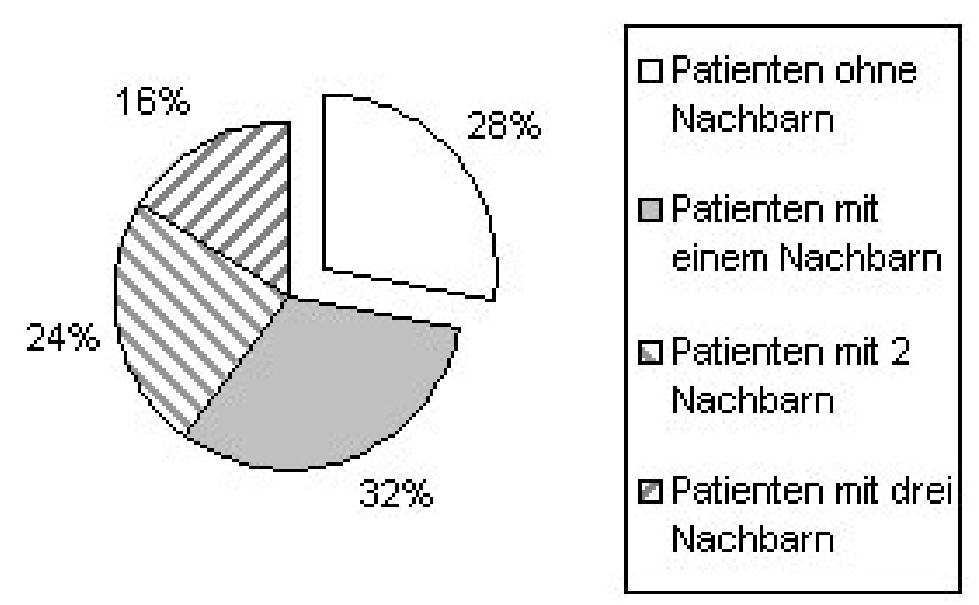

6 Patienten konnten in Einzelzimmern schlafen, 19 in Mehrbettzimmern. Sieben Patienten hatten einen Nachbarpatienten, sechs mussten das Zimmer mit 2 Nachbarn teilen und fünf Patienten hatten 3 Nachbarn.

Tabelle 3.1: Ramsay- und EPS-Score

\begin{tabular}{l|c|c|c|c||c|c|c|c} 
Ramsay-Score & 1 & 2 & 3 & $4-6$ & EPS-Score & 1 & 2 & 3 \\
\hline & 4 & 21 & 0 & 0 & & 11 & 11 & 3
\end{tabular}

Ramsay et al. (1974) unterschieden in ihren Studien 6 Grade der Sedierung. RamsayScore und EPS siehe Kapitel 2.5 (S.24) und Tabellen 2.1 (S.26) und 2.2 (S.27). 


\section{Tabelle 3.2: Ergebnisse der Schlafparameter}

\begin{tabular}{r|c|c|c|c} 
& MW & Var & Min & Max \\
\hline $\operatorname{TIB}(\mathrm{h})$ & 7,67 & 0,53 & 6,52 & 8,72 \\
SPT(h) & 6,66 & 0,98 & 4,53 & 8,65 \\
TST(h) & 3,76 & 1,57 & 1,18 & 7,58 \\
SEI(\%) & 0,49 & 0,19 & 0,17 & 0,87 \\
SK(\%) & 0,55 & 0,19 & 0,22 & 0,88 \\
SOL1(h) & 0,59 & 0,56 & 0,01 & 2,02 \\
\hline Gesamt Wach(h) & 2,91 & 2,84 & 1,07 & 5,13 \\
AWI & 4,42 & 1,81 & 0,8 & 7,0
\end{tabular}

TIB - Zeit im Bett; SPT - Schlafdauer; TST - Totale Schlafzeit; SEI- Schlafeffizienzindex; SK - Schlafkontinuität; SOL1 - Einschlaflatenz bis zum ersten Stadium S1; AWI Aufwachindex

trischen Daten, sowie Alters und Geschlechterverteilung sind in Tabelle 6.3 (Siehe Anhang S.88) nochmals zusammengefasst.

\subsection{Auswertung}

\subsubsection{Schlafparameter}

Aus Abb. 3.3 (S.44) geht hervor, dass die Schlafparameter normal verteilt sind. Die Angaben erfolgen, wenn nicht anderweitig angegeben: Mittelwert \pm Standardabweichung (Minimum Maximum). Aus den Messungen ergaben sich die in Tabelle 3.2 (S.43) dargestellten Schlafparameter.

Bei den 25 in die Auswertung eingeschlossenen Patienten betrug der mittlere Untersuchungszeitraum $7,67 \pm 0,53 \mathrm{~h}(6,52-8,72 \mathrm{~h})$. Es ergab sich eine gemittelte Schlafdauer (SPT) von $6,66 \pm 0,98 \mathrm{~h}(4,53-8,65 \mathrm{~h})$. Die totale Schlafzeit (TST) belief sich auf $3,76 \pm 1,57 \mathrm{~h}(1,18$ $-7,58 \mathrm{~h})$. Vier Messungen ergaben eine totale Schlafzeit unter 2 Stunden. Nur 3 Patienten 
Abbildung 3.3: Die Normalverteilung der Schlafparameter

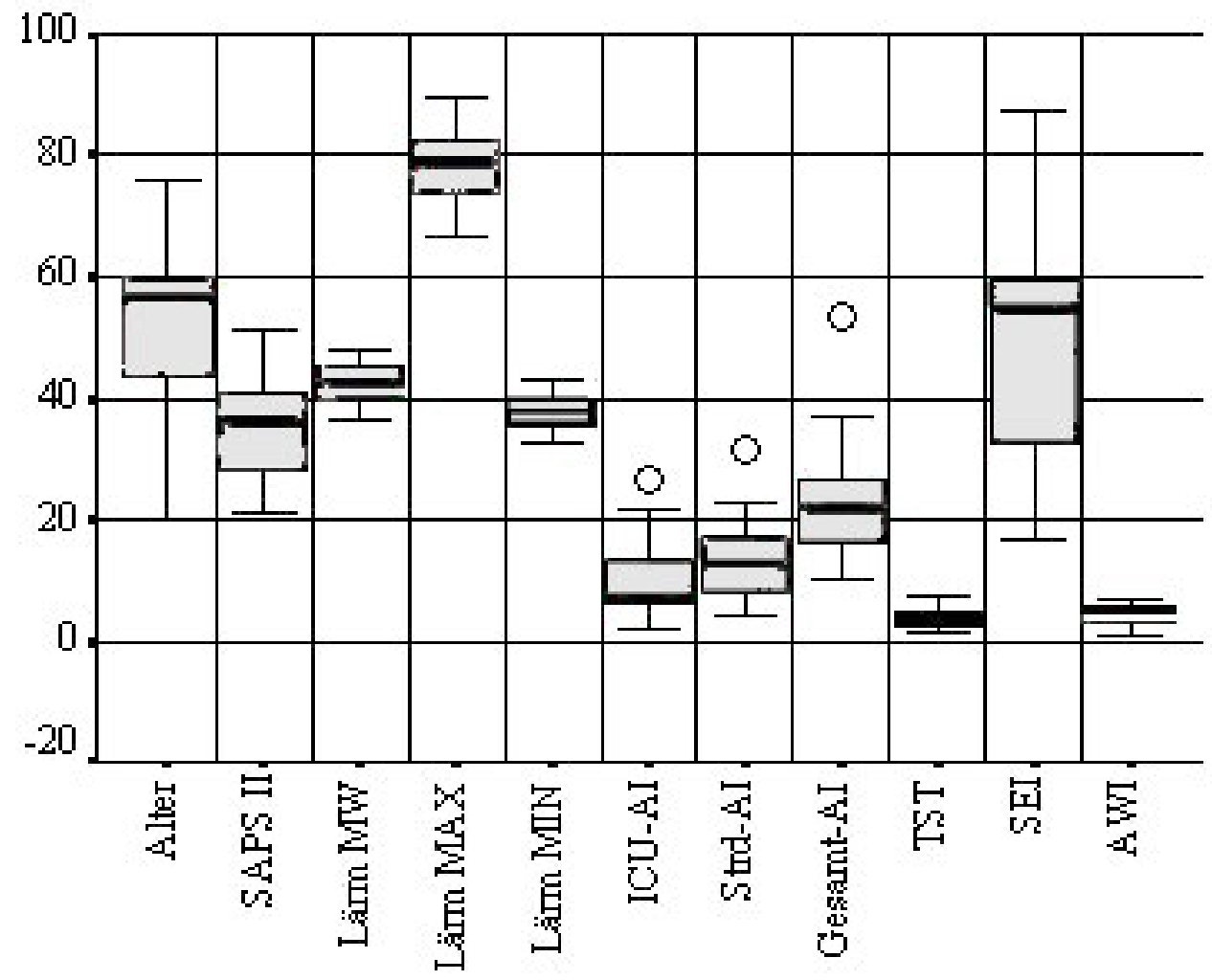

Aus der Abbildung geht die Normalverteilung der dargestellten Schlafparameter hervor. 


\section{Abbildung 3.4: Schlafstadienverteilung im Überblick}

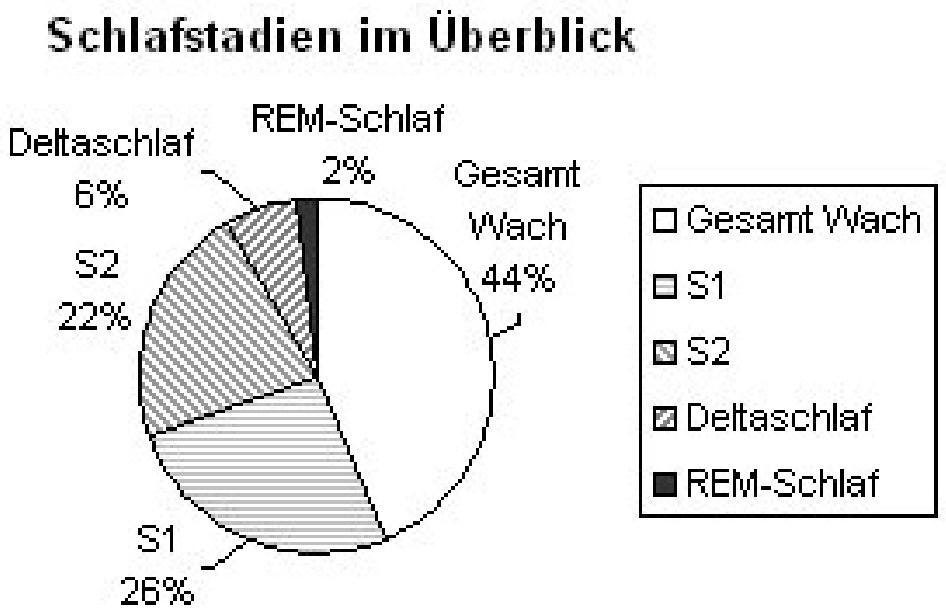

Bezogen auf die gesamte Schlafzeit TST waren die Patienten unserer Untersuchung zu $44,4 \%$ wach, die Stadien S1 und S2 traten zu 26\% bzw 22\% auf. $\delta$ - und REM-Schlaf kamen zu $6 \%$ bzw. $2 \%$ vor.

schliefen länger als 5 Stunden. Hieraus errechnete sich ein mittlerer Schlafeffizienzindex (SEI) von $0,49 \pm 0,19(0,17-0,87)$. Die Einschlaflatenz (SOL1) bezogen auf das erste Stadium S1 war durchschnittlich 35,4 $\pm 33,5$ min (0,01 - 2,02h). Der Aufwachindex betrug 4,42 $\pm 1,81$ pro Stunde $(0,8-7,0)$.

Die prozentuale Verteilung der Schlafstadien bezogen auf die SPT sah wie folgt aus: Während der Untersuchung (SPT) waren die Patienten zu 44,67 $\pm 18,65 \%$ wach. S1 und S2 waren mit je 25,7 $\pm 15,44 \%$ und 21,65 $\pm 16,47 \%$ beteiligt. Der Anteil des $\delta$-Schlafs belief sich auf 6,02 $\pm 11,18 \%$ und REM-Schlaf auf 1,96 $\pm 3,40 \%$ (Abb. 3.4 S.45).

Bei 17 von 25 Messungen trat kein REM-Schlaf auf. Bei keiner Messung war der REM-Anteil im Normbereich, also über 20\%; nur eine Messung lag über 10\%. $\delta$-Schlaf konnte bei acht Messungen nicht festgestellt werden, nur 2 waren im Normbereich ( $>15 \%)$, achtzehn von 25 Messungen wiesen weniger als 5\% $\delta$-Schlaf auf. Vergleichend zeigen die Abbildungen 4.1 (S.70) die 'normalen' Schlafparameter eines Gesunden und Abb. 1.1 (S.5) die Werte eines normalen Schlafes. In den Abbildungen 3.3 und 3.4(S.46 und 47) sind die wichtigsten Schlafparameter, die Aufteilung der Standard und ICU-Arousals, sowie die Alters und Geschlechterverteilung 
Tabelle 3.3: Schlafparameter aller Messungen A

\begin{tabular}{|c|c|c|c|c|c|c|c|c|c|c|c|c|c|c|c|}
\hline Messung & $\mathrm{A}$ & B & $\mathrm{C}$ & $\mathrm{D}$ & $\mathrm{E}$ & $\mathrm{F}$ & G & H1a & I & $\mathrm{J} 2 \mathrm{a}$ & K & $\mathrm{L}$ & M & N3b & $\mathrm{O} 4 \mathrm{a}$ \\
\hline Alter & 50 & 20 & 43 & 60 & 50 & 29 & 32 & 71 & 74 & 59 & 56 & 44 & 70 & 76 & 50 \\
\hline sex & $\mathrm{w}$ & $\mathrm{w}$ & $\mathrm{m}$ & $\mathrm{m}$ & $\mathrm{m}$ & $\mathrm{w}$ & $\mathrm{w}$ & $\mathrm{w}$ & $\mathrm{w}$ & $\mathrm{m}$ & $\mathrm{m}$ & $\mathrm{m}$ & $\mathrm{m}$ & $\mathrm{m}$ & $\mathrm{m}$ \\
\hline Nachb & 2 & 1 & 0 & 1 & 2 & 0 & 3 & 2 & 1 & 1 & 3 & 1 & 2 & 0 & 1 \\
\hline EPS & 3 & 2 & 1 & 1 & 2 & 1 & 2 & 2 & 1 & 1 & 3 & 3 & 2 & 1 & 2 \\
\hline TIB & 7,4 & 8,7 & 6,5 & 7,7 & 7,5 & 7,7 & 8,0 & 7,6 & 7,6 & 8,0 & 7,1 & 7,7 & 8,3 & 8,3 & 7,8 \\
\hline SPT & 7,3 & 8,7 & 5,8 & 7,2 & 5,2 & 6,9 & 6,6 & 6,5 & 6,7 & 6,1 & 6,4 & 7,5 & 8,0 & 6,9 & 4,5 \\
\hline $\mathrm{TST}$ & 5,5 & 7,6 & 3,8 & 4,4 & 3,3 & 3,6 & 2,2 & 3,5 & 4,5 & 2,9 & 1,6 & 4,7 & 4,9 & 2,7 & 2,4 \\
\hline SEI & 74,3 & 87,2 & 58,2 & 56,7 & 43,8 & 46,8 & 27,2 & 46,3 & 59,5 & 36,2 & 22,3 & 61,5 & 59,1 & 32,4 & 30,4 \\
\hline AWI & 5,4 & 2,3 & 6,2 & 6,4 & 3,2 & 2,2 & 2,7 & 4,3 & 4,5 & 5,5 & 5,9 & 3,5 & 6,0 & 7,0 & 6,6 \\
\hline $\mathrm{W} \%$ & 24,9 & 12,3 & 34,6 & 39,7 & 37,5 & 47,8 & 67,4 & 45,6 & 32,0 & 52,7 & 75,3 & 37,3 & 38,6 & 61,1 & 47,7 \\
\hline $\mathrm{S} 1 \%$ & 26,0 & 12,3 & 25,0 & 51,3 & 13,5 & 11,1 & 20,2 & 51,4 & 54,6 & 42,3 & 24,7 & 22,6 & 55,6 & 20,8 & 25,6 \\
\hline $\mathrm{S} 2 \%$ & 46,9 & 56,6 & 26,4 & 9,0 & 29,7 & 20,8 & 12,5 & 2,8 & 13,3 & 5,0 & 0,0 & 38,8 & 5,7 & 17,4 & 23,8 \\
\hline$\delta \%$ & 2,2 & 6,1 & 13,9 & 0,0 & 10,2 & 15,8 & 0,0 & 0,0 & 0,1 & 0,0 & 0,0 & 1,3 & 0,0 & 0,7 & 2,9 \\
\hline REM \% & 0,0 & 12,6 & 0,0 & 0,0 & 8,9 & 4,6 & 0,0 & 0,0 & 0,0 & 0,0 & 0,0 & 0,0 & 0,0 & 0,0 & 0,0 \\
\hline Schall MW dB & 42,0 & 47,8 & 36,6 & 47,0 & 45,1 & 38,4 & 39,0 & 41,5 & 41,8 & 42,5 & 45,2 & 44,1 & 41,3 & 40,0 & 47,6 \\
\hline undef. LA/h & 4,8 & 2,5 & 4,2 & 1,2 & 13,2 & 4,4 & 19,4 & 4,8 & 7,3 & 9,7 & 15,7 & 8,3 & 6,5 & 9,3 & 6,3 \\
\hline Monitor/h & 1,3 & 0,1 & 0,0 & 0,0 & 2,5 & 0,3 & 0,5 & 0,0 & 5,3 & 3,5 & 8,2 & 2,8 & 2,2 & 0,7 & 0,0 \\
\hline Standard-A./h & 18,9 & 16,8 & 15,3 & 16,7 & 4,3 & 9,7 & 31,5 & 15,0 & 20,7 & 12,4 & 7,5 & 11,0 & 5,5 & 9,3 & 13,5 \\
\hline ICU-Arousal/h & 7,5 & 6,3 & 5,0 & 2,1 & 17,4 & 6,1 & 21,8 & 5,7 & 16,1 & 13,1 & 26,4 & 11,2 & 10,6 & 11,5 & 8,0 \\
\hline Personal/h & 0,5 & 3,3 & 0,3 & 0,5 & 0,0 & 0,8 & 0,0 & 0,9 & 1,5 & 0,0 & 0,0 & 0,0 & 1,0 & 0,4 & 1,3 \\
\hline Nachbar/h & 0,0 & 2,0 & 0,0 & 0,0 & 0,0 & 0,0 & 2,0 & 0,0 & 1,0 & 0,0 & 0,0 & 0,0 & 0,0 & 2,0 & 0,0 \\
\hline $\mathrm{RR} / \mathrm{h}$ & 0,4 & 0,1 & 0,3 & 0,0 & 1,5 & 0,6 & 0,5 & 0,0 & 1,1 & 0,0 & 1,3 & 0,0 & 0,2 & 0,4 & 0,0 \\
\hline Perfusor/h & 0,5 & 0,0 & 0,3 & 0,0 & 0,3 & 0,0 & 0,5 & 0,0 & 0,7 & 0,0 & 1,3 & 0,0 & 0,6 & 0,0 & 0,4 \\
\hline sonstige A./h & 0,0 & 0,0 & 0,0 & 0,5 & 0,0 & 0,0 & 0,0 & 0,0 & 0,0 & 0,0 & 0,0 & 0,2 & 0,0 & 0,0 & 0,0 \\
\hline A.Gesamt/h & 26,4 & 23,1 & 20,3 & 18,8 & 21,7 & 15,8 & 53,3 & 20,7 & 36,8 & 25,5 & 33,9 & 22,3 & 16,1 & 20,7 & 21,6 \\
\hline
\end{tabular}

Nachb - Nachbarn; Wachep - Wachepisode; $\delta$ - Deltaschlaf; Standard-A.- Standard-

Arousal; undef. LA - undefiniertes Lärm-Arousal; A.Gesamt - Arousals Gesamt.

der Messungen aufgelistet. Als Beispiel diene hier Patientin Frau G, 32 Jahre alt, die neben 3 Nachbarn schlief. Von 8 Stunden im Bett, schlief sie innerhalb von 6,6 Stunden (SPT) nur 2,2 Stunden (TST). Die Schlafeffizienz war somit 27,2\%, folglich war Frau G zu 67,4\%, also mehr als 2/3 der Schlafenszeit wach. Die Schlafstadien S1 und S2 traten zu 11,1\% respektive $12,5 \%$ auf, REM- und $\delta$-Schlaf traten nicht auf. Bei einem mittleren Schallpegel von $39 \mathrm{~dB}$ kamen durchschnittlich 53,3 Arousals pro Stunde vor, 21,8 hiervon waren ICU- und 31,5 Standard-Arousals. Die häufigsten Arousals waren undefinierte Lärm-Arousals.

\subsubsection{Ergebnisse der Lärmmessung}

Der Geräuschpegel betrug im Mittel 42,94 $\pm 3,08 \mathrm{~dB}$, das Maximum im Mittel 85,45 $\pm 12,64 \mathrm{~dB}$ und Minimum 36,56 $\pm 3,91 \mathrm{~dB}$, es konnten Schallspitzen bis 95,5 dB gemessen werden. Nur 
Tabelle 3.4: Schlafparameter aller Messungen B

\begin{tabular}{|c|c|c|c|c|c|c|c|c|c|c|c|c|c|c|c|}
\hline Messung & $\mathrm{P}$ & $\mathrm{Q}$ & R5a & $\mathrm{S} 5 \mathrm{~b}$ & $\mathrm{~T} 6 \mathrm{a}$ & $\mathrm{U} 6 \mathrm{~b}$ & $\mathrm{~V} 7 \mathrm{a}$ & W7b & $\mathrm{x}$ & $\mathrm{Y}$ & MW & Med & $\operatorname{Max}$ & Min & $\sigma$ \\
\hline Alter & 65 & 59 & 60 & 60 & 56 & 56 & 28 & 28 & 36 & 70 & 52 & 56 & 76 & 20 & 15,9 \\
\hline sex & $\mathrm{m}$ & $\mathrm{m}$ & $\mathrm{m}$ & $\mathrm{m}$ & $\mathrm{m}$ & $\mathrm{m}$ & $\mathrm{m}$ & $\mathrm{m}$ & $\mathrm{m}$ & $\mathrm{w}$ & & & & & \\
\hline Nachb & 0 & 2 & 3 & 3 & 0 & 0 & 1 & 2 & 1 & 0 & & & & & \\
\hline EPS & 1 & 2 & 1 & 2 & 1 & 1 & 2 & 2 & 2 & 1 & & & & & \\
\hline TIB & 7,3 & 7,6 & 8,7 & 8,1 & 7,4 & 6,8 & 8,0 & 7,1 & 7,7 & 7,2 & 7,7 & 7,7 & 8,7 & 6,5 & 0,5 \\
\hline $\mathrm{SPT}$ & 6,6 & 6,3 & 8,1 & 7,6 & 7,1 & 6,7 & 6,8 & 4,9 & 5,4 & 6,7 & 6,7 & 6,7 & 8,7 & 4,5 & 1,0 \\
\hline $\mathrm{TST}$ & 1,4 & 1,4 & 6,1 & 4,8 & 4,4 & 4,4 & 3,6 & 1,2 & 4,2 & 5,0 & 3,8 & 3,8 & 7,6 & 1,2 & 1,6 \\
\hline SEI & 19,6 & 18,1 & 70,4 & 59,1 & 58,8 & 64,6 & 45,1 & 16,6 & 54,4 & 70,2 & 48,8 & 54,4 & 87,2 & 16,6 & 19,2 \\
\hline AWI & 5,8 & 6,2 & 5,8 & 0,8 & 1,8 & 3,6 & 3,2 & 2,3 & 3,1 & 6,2 & 4,4 & 4,5 & 7,0 & 0,8 & 1,8 \\
\hline $\mathrm{W} \%$ & 78,3 & 78,1 & 24,7 & 37,3 & 38,7 & 34,6 & 47,1 & 75,7 & 23,4 & 24,2 & 44,7 & 38,7 & 78,3 & 12,3 & 18,7 \\
\hline $\mathrm{S} 1 \%$ & 13,1 & 21,4 & 10,4 & 1,2 & 20,6 & 21,5 & 29,3 & 7,7 & 16,7 & 43,5 & 25,7 & 21,5 & 55,6 & 1,2 & 15,4 \\
\hline $\mathrm{S} 2 \%$ & 7,8 & 0,5 & 41,7 & 10,1 & 34,5 & 31,9 & 16,9 & 13,4 & 53,9 & 21,9 & 21,6 & 17,4 & 56,6 & 0,0 & 16,5 \\
\hline$\delta \%$ & 0,9 & 0,0 & 23,2 & 51,4 & 3,0 & 7,3 & 0,0 & 3,3 & 0,8 & 7,3 & 6,0 & 1,3 & 51,4 & 0,0 & 11,2 \\
\hline REM \% & 0,0 & 0,0 & 0,0 & 0,0 & 3,2 & 4,7 & 6,7 & 0,0 & 5,2 & 3,1 & 2,0 & 0,0 & 12,6 & 0,0 & 3,4 \\
\hline Schall MW dB & 40,3 & 45,3 & 46,0 & 44,6 & 44,8 & 39,3 & 44,5 & 42,0 & 46,3 & 40,5 & 42,9 & 42,5 & 47,8 & 36,6 & 3,1 \\
\hline undef. LA/h & 0,0 & 11,6 & 2,5 & 1,7 & 2,3 & 3,9 & 6,7 & 8,5 & 5,5 & 6,6 & 6,7 & 6,3 & 19,4 & 0,0 & 4,6 \\
\hline Monitor/h & 0,0 & 3,6 & 2,1 & 1,7 & 2,1 & 1,4 & 0,0 & 1,7 & 0,0 & 0,4 & 1,6 & 1,3 & 8,2 & 0,0 & 2,0 \\
\hline Standard A./h & 22,5 & 14,6 & 9,3 & 4,8 & 19,9 & 8,9 & 19,7 & 6,8 & 7,7 & 7,1 & 13,2 & 12,4 & 31,5 & 4,3 & 6,6 \\
\hline ICU-Arousal/h & 8,4 & 18,9 & 6,2 & 5,5 & 4,6 & 6,8 & 7,2 & 14,4 & 5,5 & 7,5 & 10,2 & 7,5 & 26,4 & 2,1 & 6,0 \\
\hline Personal/h & 5,6 & 0,0 & 0,2 & 0,6 & 0,0 & 0,9 & 0,0 & 0,0 & 0,0 & 0,0 & 0,7 & 0,3 & 5,6 & 0,0 & 1,3 \\
\hline Nachbar/h & 0,0 & 1,0 & 0,3 & 0,2 & 0,2 & 0,0 & 0,0 & 1,7 & 0,0 & 0,2 & 0,4 & 0,0 & 2,0 & 0,0 & 0,7 \\
\hline $\mathrm{RR} / \mathrm{h}$ & 0,0 & 1,5 & 0,5 & 0,4 & 0,0 & 0,7 & 0,0 & 0,8 & 0,0 & 0,2 & 0,4 & 0,3 & 1,5 & 0,0 & 0,5 \\
\hline Perfusor $/ \mathrm{h}$ & 0,7 & 0,7 & 0,5 & 0,4 & 0,0 & 0,0 & 0,6 & 1,7 & 0,0 & 0,2 & 0,4 & 0,3 & 1,7 & 0,0 & 0,4 \\
\hline sonstige A./h & 2,8 & 0,7 & 0,2 & 0,4 & 0,0 & 0,0 & 0,0 & 0,0 & 0,0 & 0,0 & 0,2 & 0,0 & 3,3 & 0,0 & 0,7 \\
\hline A.Gesamt/h & 30,9 & 33,5 & 15,5 & 10,3 & 24,5 & 15,7 & 26,9 & 19,4 & 13,2 & 14,7 & 23,3 & 21,6 & 53,3 & 10,3 & 9,2 \\
\hline
\end{tabular}

Nachb - Nachbarn; Wachep - Wachepisode; $\delta$ - Deltaschlaf; Standard A.- Standard-

Arousal; undef. LA - undefiniertes Lärm-Arousal; A.Gesamt - Arousals Gesamt; MW -

Mittelwert; Med - Median; Min - Minimum; Max - Maximum; $\sigma$ - Standardabweichung. 


\section{Tabelle 3.5: Ergebnisse der Schallmessung}

\begin{tabular}{r|c|c|c|c} 
& MW & Var & Min & Max \\
\hline Schall MW(dB) & 42,94 & 3,08 & 36,60 & 47,80 \\
Schall MAX(dB) & 85,45 & 12,64 & 69,30 & 95,50 \\
Schall MIN(dB) & 36,56 & 3,91 & 26,50 & 41,80
\end{tabular}

MW-Mittelwert; Var- Varianz, Standardabweichung; MAX-Maximum; MIN-Minimum

einmal wurde ein Schallminimum von $26,5 \mathrm{~dB}$ gemessen.

\subsubsection{Ergebnisse der Arousals}

Bezogen auf alle ausgewerteten Messungen ergab sich die Aufteilung der Gesamtzahl aller Arousals pro Stunde wie folgt: 13,16/h (55,9\%) waren Standard-Arousal und 10,38/h $(44,1 \%)$ ICU-Arousals. Um einen Vergleich mit der Untersuchung Freedman et al. herstellen zu können, wurde eine zweite Einteilung in akustische und non-akustische Arousals vorgenommen: Dabei ergab sich, dass 9,95/h (42,2\%) akustischer und 13,60/h (57,8\%) non-akustischer Herkunft waren (Siehe Abb. 3.5 S.49). Zunächst stellt man die ICU- den Standard-Arousal gegenüber.

\subsubsection{Standard-Arousals - ICU-Arousals}

Die Verteilung der Arousals war folgendermaßen:

Atemabhängige Arousals, bedingt durch Hypopnoe, Apnoe, Entsättigung oder durch Schnarchen betragen 2,14 $\pm 6,59 / \mathrm{h}$ entsprechend 9,1\% aller Arousals. 0,9\% (0,21 $\pm 0,67$ Arousal/h) werden durch Bewegung oder periodisch wiederkehrende Bewegungen hervorgerufen. 45,9\% sind spontane Arousals, durchschnittlich 13,17 \pm 6,61 Arousals pro Stunde. 44,1\% aller Arousals sind ICU-Arousals entsprechend 10,15 \pm 6,01/h (Abb. 3.6 S. 50).

Siehe auch Tabelle der ICU-Arousals 2.6 (S.34) und Standardarousal 1.1 (S.8). In der Sparte der ICU-Arousals ist das undefinierte Lärm-Arousal mit 6,66 Arousal/h (28,3\%) am häufigsten. Als Ursache kommen hier alle akustischen Ereignisse in Frage. Alle genannten Ursachen 


\section{Abbildung 3.5: Alle Arousals im Überblick}
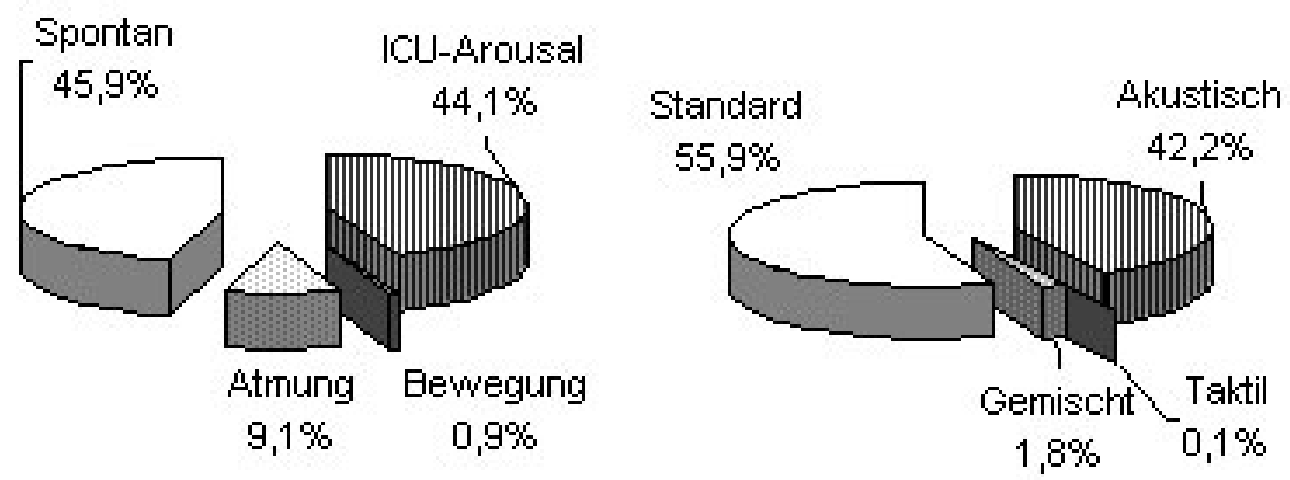

Bezogen auf alle ausgewerteten Messungen ergab sich die Aufteilung der Gesamtzahl aller Arousals pro Stunde wie folgt: 13,16/h (55,9\%) waren Standard-Arousal und 10,38/h $(44,1 \%)$ ICU-Arousals. Um einen Vergleich mit der Untersuchung Freedman et al. herstellen zu können, wurde eine zweite Einteilung in akustische und non-akustische Arousals vorgenommen: Dabei ergab sich, dass 9,95/h (42,2\%) akustischer und 13,60/h (57,8\%) non-akustischer Herkunft waren.

könnten diese Arousals bedingt haben. Von den Arousals, deren Ursache benannt werden konnte, ist der Monitor, der die Vitalparameter des Patienten kontrolliert mit 1,61 Arousals pro Stunde, 6,8\% entsprechend, der häufigste Auslöser eines Arousals. Gefolgt vom Personal, das im Schnitt 0,71 Arousals pro Stunde (3,0\%) auslöst. Weiter folgen der Nachbarpatient mit 0,43 (1,8\%), das automatische Blutdruckmessgerät mit 0,41 (1,8\%) und der Perfusor mit 0,37 Arousals pro Stunde, 1,6\% entsprechend. Selten wurde das Telefon-Arousal mit 0,4\% (0,09 Arousal/h), das Beatmungsgerät eines Nachbarn mit 0,3\% (0,08 Arousal/h) oder eine Intervention am Patienten mit 0,1\%(0,03 Arousal/h) beobachtet. Insgesamt ergab sich ein Standard-Arousalindex von $13,17 / \mathrm{h} \pm 6,61$ und ein ICU-Arousalindex von 10,15/h $\pm 6,01$. Also sind 55,9\% der Arousals Standard-Arousal und 44,1\% ICU-Arousals bei einem GesamtArousalindex von 23,26/h $\pm 9,18$. Das heißt, dass ein Patient in einer Untersuchung mehr als 23 mal pro Stunde im Schlaf gestört wurde. Dies entspricht einer dreiminütigen Weckreaktion. 


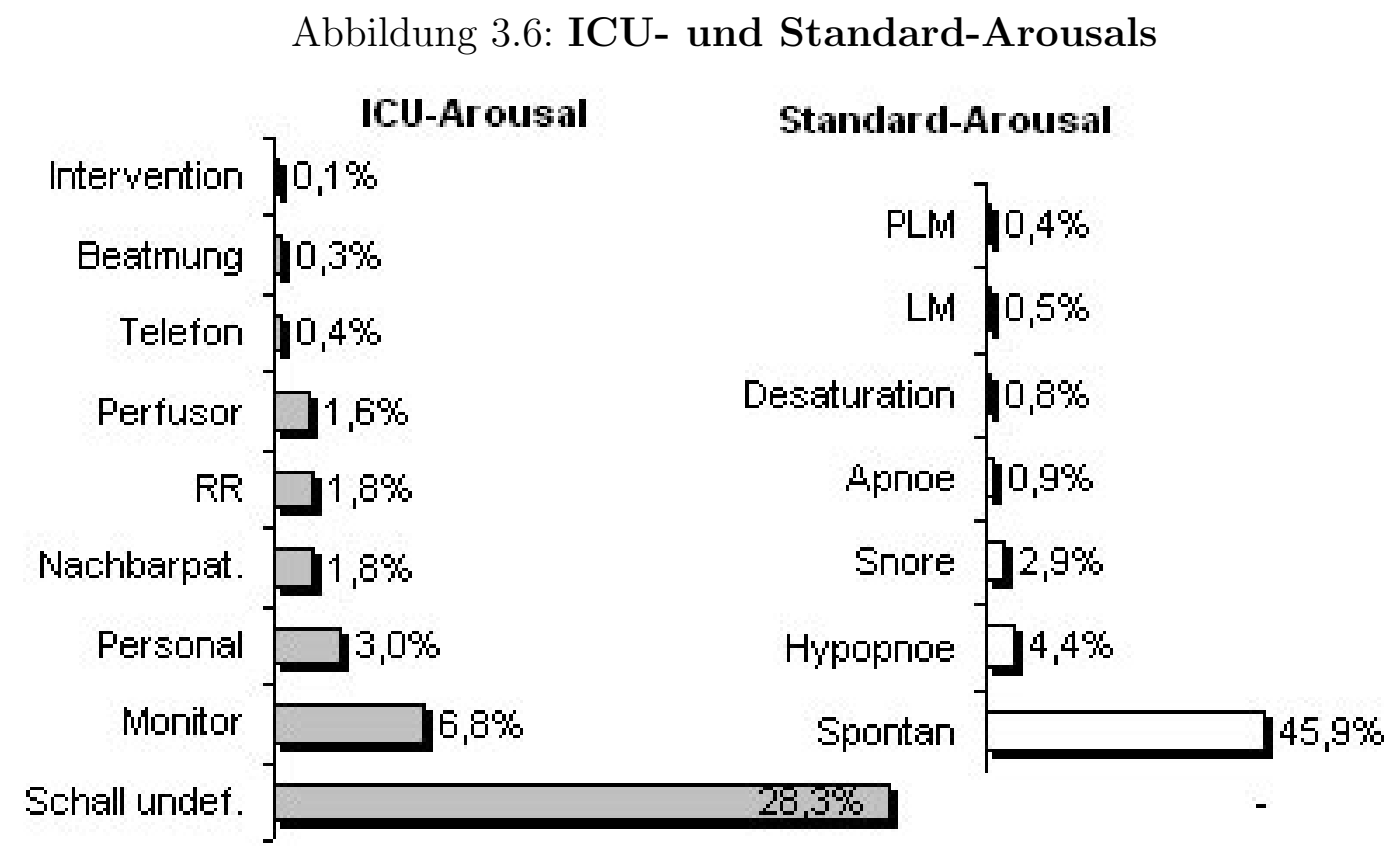

Atemabhängige Arousals, bedingt durch Hypopnoe, Apnoe, Entsättigung oder durch Schnarchen betragen 2,14 $\pm 6,59 / \mathrm{h}$ entsprechend 9,1\% aller Arousals. 0,9\% (0,21 \pm $0,67 / \mathrm{h}$ ) werden durch Bewegung oder periodisch wiederkehrende Bewegungen hervorgerufen. 45,9\% sind spontane Arousals, durchschnittlich 13,17 \pm 6,61 Arousals pro Stunde. $44,1 \%$ aller Arousals sind ICU-Arousals entsprechend 10,15 $\pm 6,01 / \mathrm{h}$. Die undefinierten Schall- und Monitor-Arousals sind die häufigsten ICU-Arousals. 


\begin{tabular}{|c|c|c|}
\hline & $\mathrm{MW}(/ \mathrm{h})$ & Anteil $(\%)$ \\
\hline akustische Arousals & 9,95 & 42,2 \\
\hline Schall undef. & 6,66 & 28,3 \\
\hline Monitor & 1,61 & 6,8 \\
\hline Personal & 0,71 & 3,0 \\
\hline Nachbarpat. & 0,43 & 1,8 \\
\hline Perfusor & 0,37 & 1,6 \\
\hline Telefon & 0,09 & 0,4 \\
\hline Beatmung & 0,08 & 0,3 \\
\hline nicht-akustische Arousals & 13,60 & 57,8 \\
\hline Standard-AI & 13,17 & 55,9 \\
\hline $\mathrm{RR}$ & 0,41 & 1,8 \\
\hline Intervention & 0,03 & 0,1 \\
\hline Gesamt & 23,54 & 100 \\
\hline
\end{tabular}

Es waren 42,2\% akustische und 57,8\% nicht-akustische Arousals zu beobachten, entsprechend einem Arousalindex von 9,95/h für akustische und 13,60/h für nicht-akustische Arousals. Das häufigste akustische Arousal war der undefinierte Lärm mit 6,66 Arousals/h respektive $28,3 \%$

\subsubsection{Akustische und nicht-akustische Arousals}

Es waren 42,2\% akustische und 57,8\% nicht-akustische Arousals zu beobachten, entsprechend einem Arousalindex von 9,95/h für akustische und 13,60/h für nicht-akustische Arousals. Die Tabelle 3.6 (S. 51) soll die Häufigkeitsverhältnisse der akustischen und nicht akustischen Arousals verdeutlichen. Deshalb ist der absolute und der relative Wert jeder Arousalkategorie angegeben.

Die akustischen Arousals beinhalten das Arousal des undefinierten Lärms mit 28,3\% (6,66/h). Gefolgt von Monitor-, Personal-, Nachbarpatient- und Perfusor-Arousals, mit 6,8\%, 3\%, 1,8\% und 1,6\%. Den geringsten Anteil machen das Telefon- und das Beatmungs-Arousal mit $0,4 \%$ und $0,3 \%$ der akustischen Arousals aus. Die nicht-akustischen Arousals bestehen aus Standard-(55,9\%), Blutdruck-(1,8\%) und Interventions-Arousals(0,1\%). Die Ergebnisse der akustischen und nicht-akustischen Arousals werden im Kapitel 4.5.5 (S.73) mit den Ergebnissen von Freedman et al. (2001) verglichen. 


\subsubsection{Lärmpegel und Arousalprofile}

Das Messintervall war in 4 Abschnitte von jeweils 2 Stunden eingeteilt (Siehe Kapitel 2.8.3 und 2.8.5 S.38). Im zeitlichen Verlauf wurden Schallmittelwert, -maximum und -minimum, der Gesamt-Arousalindex sowie die ICU- und Standard-Arousalindices, der prozentuale Anteil des ICU-Arousalindex, als auch die totale Schlafzeit (TST) und der Aufwachindex (AWI) dargestellt (Siehe Abbildung 3.7 S.53). Der Schallmittelwert ist zu den Zeitpunkten Eins und Vier mit 44,8 dB und 44,0 dB im Vergleich zu den Zeitpunkten 2 und 3 mit jeweils 44,3 dB und 43,4 dB leicht erhöht (Abb. 3.7).

In Abbildung 3.8 (S.54) sind der Standard-Arousalindex, der ICU-Arousalindex, der GesamtArousalindex sowie der Anteil der ICU-Arousals zu den Zeitpunkten Eins bis Vier aufgetragen. Der Standard-Arousalindex nimmt wie der Gesamt-Arousalindex von den Zeitpunkten Eins bis Vier, von 12,3 über 11,6 und 9,8 bis auf 5,3 Arousals pro Stunde ab. Der ICUArousalindex ist zu den Zeitpunkten Eins und Vier mit 9,4 und 6,8 Arousals pro Stunde gegenüber den Zeiten Zwei und Drei mit 7,3 und 5,9 Arousals pro Stunde erhöht.

Der Anteil des ICU-Arousalindex der Gesamt-Arousals ist zu den Zeiten 1 und 4 mit 47,7\% und 55,6\% im Vergleich zu den Intervallen 2 und 3 mit 44,1\% und 41,2\% deutlich erhöht. Trägt man den Aufwachindex (AWI) und die Totale Schlafzeit (TST) gegen die Zeit auf, so lässt sich feststellen, dass die totale Schlafzeit innerhalb eines Zeitintervalls von zwei Stunden zu den Zeiten Zwei und Drei mit 74,0 und 68,5 Minuten pro 2 Stunden höher als zu den Zeiten Eins und Vier mit 51,0 und 44,0 Minuten ist.

Das bedeutet, dass die Patienten zu den Zeitpunkten Eins und Vier nur 51 bzw. 44 Minuten innerhalb von 2 Stunden, aber zu den Zeitpunkten Zwei und Drei immerhin 74 bzw 68,5 Minuten schlafen können. Auch der Aufwachindex (AWI) ist zu den Zeitpunkten Eins und Vier mit 10,4 und 8,5 Wachphasen pro Stunde höher als zu den Zeiten Zwei und Drei mit 7,6 und 6,4 Wachphasen pro Stunde. 
Abbildung 3.7: Zeit vs Schallmittelwert -maximum und -minimum

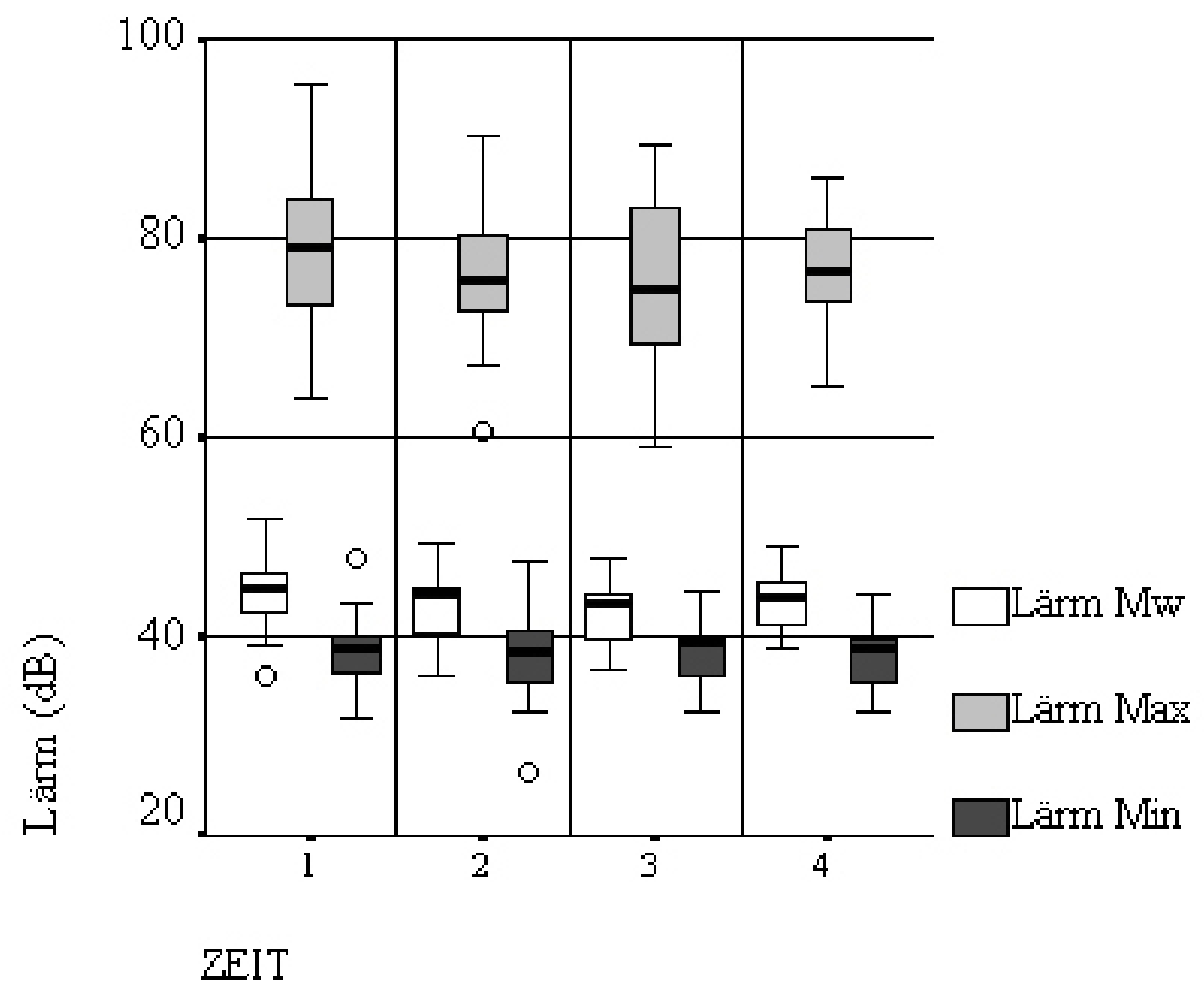

Der Schallmittelwert ist zu den Zeitpunkten Eins und Vier mit 44,8 dB und 44,0 dB im Vergleich zu den Zeitpunkten 2 und 3 mit jeweils 44,3 dB und 43,4 dB leicht erhöht. 
Abbildung 3.8: Zeit vs Standard- und ICU-Arousalindex

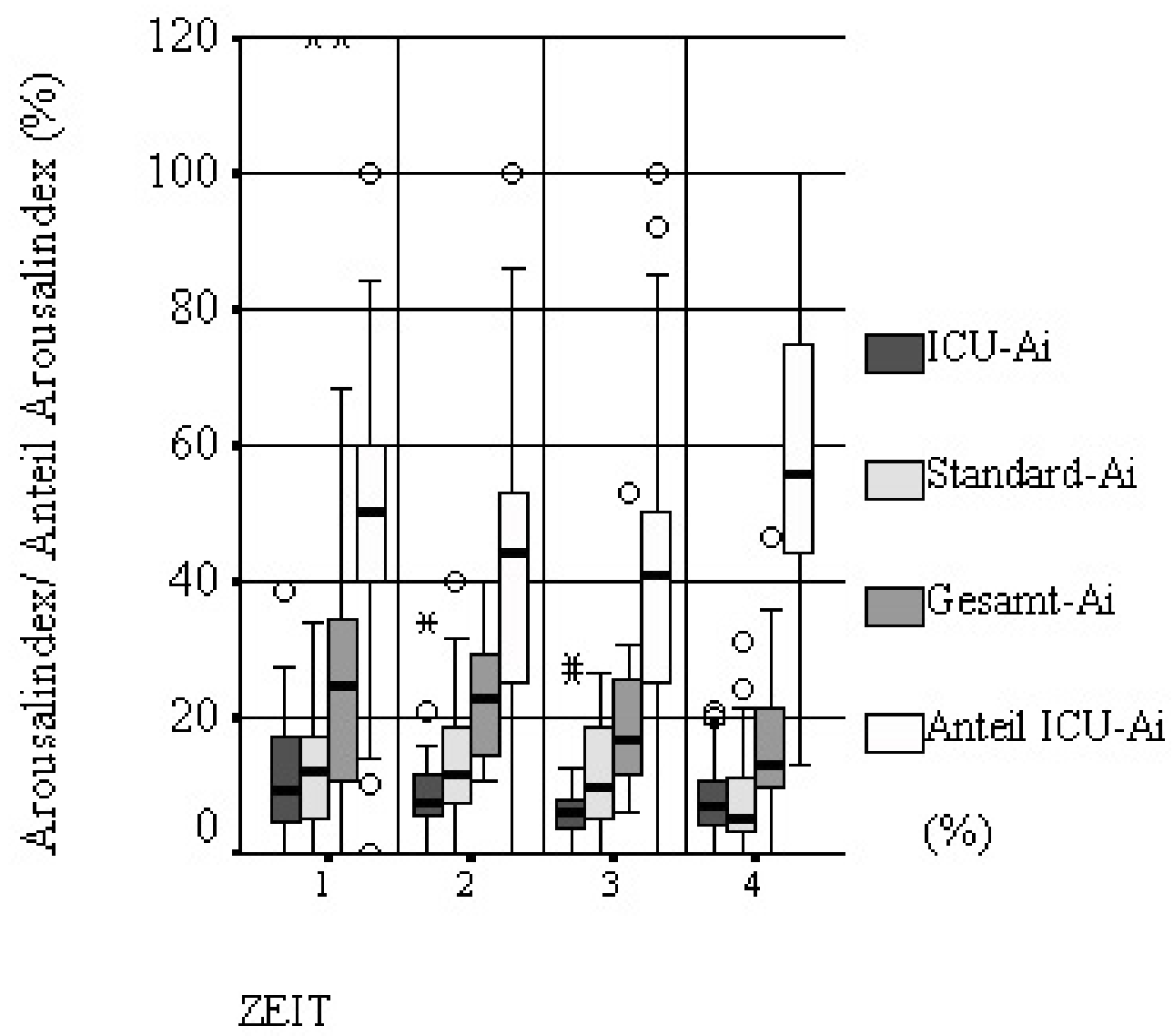

Standard-Arousalindex, der ICU-Arousalindex, der Gesamt-Arousalindex sowie der Anteil der ICU-Arousals aufgetragen zu den Zeitabschnitten 1 bis 4 . Der StandardArousalindex nimmt wie der Gesamt-Arousalindex von den Zeitpunkten Eins bis Vier, von 12,3 über 11,6 und 9,8 bis auf 5,3 Arousals pro Stunde ab. Der ICU-Arousalindex ist zu den Zeitpunkten Eins und Vier mit 9,4 und 6,8 Arousals pro Stunde gegenüber den Zeiten Zwei und Drei mit 7,3 und 5,9 Arousals pro Stunde erhöht. 
Abbildung 3.9: Zeit vs AWI und TST

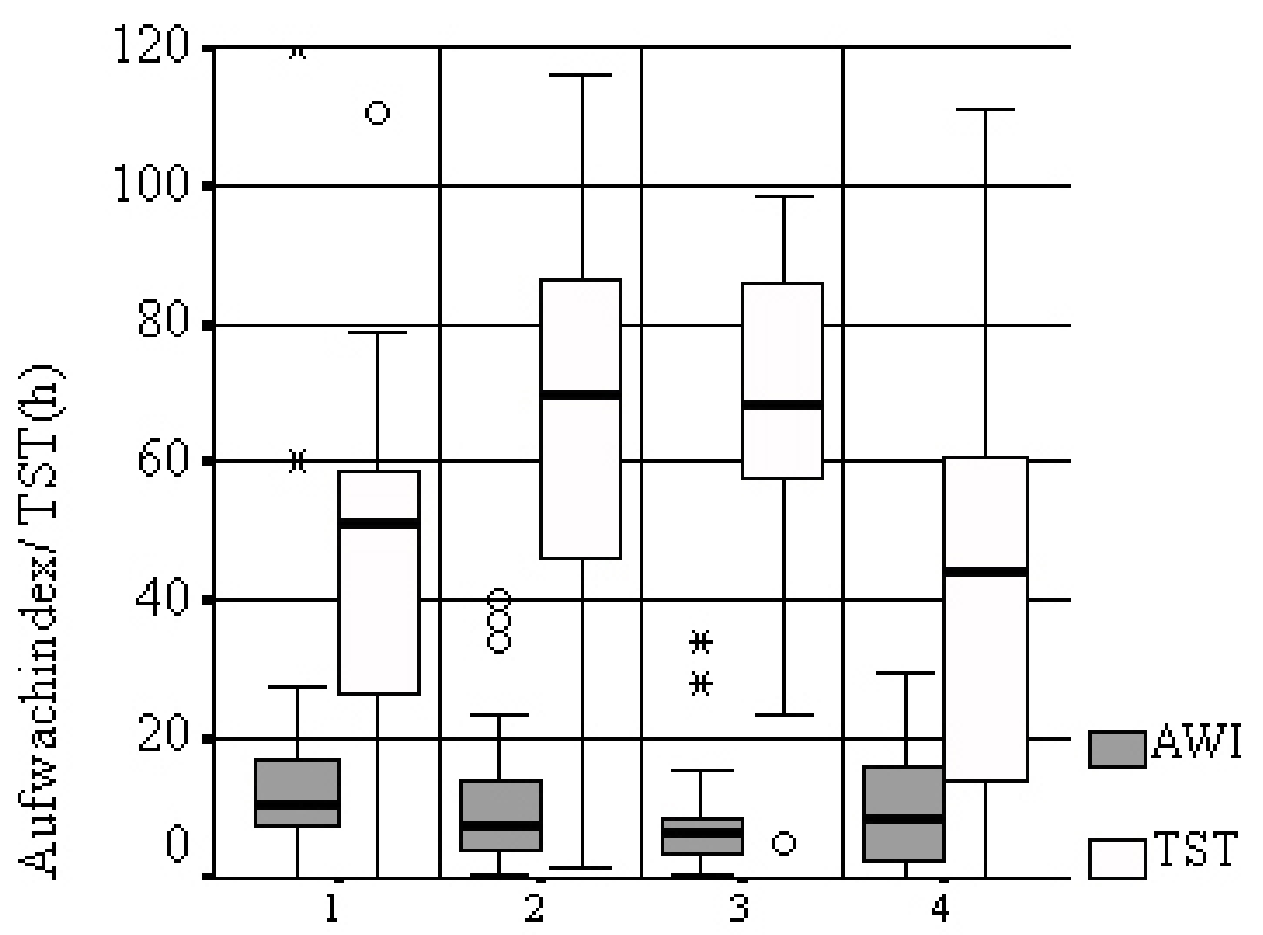
ZEIT

Die Totale Schlafzeit (TST) ist innerhalb eines Zeitintervalls von zwei Stunden zu den Zeiten Zwei und Drei mit 74,0 und 68,5 Minuten pro 2 Stunden höher als zu den Zeiten Eins und Vier mit 51,0 und 44,0 Minuten erhöht. Auch der Aufwachindex (AWI) ist zu den Zeitpunkten Eins und Vier mit 10,4 und 8,5 Wachphasen pro Stunde höher als zu den Zeiten Zwei und Drei mit 7,6 und 6,4 Wachphasen pro Stunde. 


\section{Tabelle 3.7: Korrelation des Anteils der ICU-Arousals}

\begin{tabular}{r|r|l|r|l|} 
Korrelation & Spearman-Rho & Signifikanz & Pearson & Signifikanz \\
\hline Ant ICU vs TST & $-0,232$ & 0,132 & $-0,36$ & $\mathbf{0 , 0 3 8 5}$ \\
\hline Ant ICU vs SEI & $-0,269$ & $\mathbf{0 , 0 2 9}$ & $-0,385$ & $\mathbf{0 , 0 2 9}$ \\
AWI vs Schallmax & 0,061 & 0,386 & 0,108 & 0,304 \\
Ant ICU vs Schall MW & $-0,024$ & 0,445 & 0,006 & 0,489 \\
Ant ICU vs Schall Max & 0,072 & 0,366 & 0,096 & 0,323 \\
\hline Ant ICU vs Nachbarn & 0,397 & $\mathbf{0 , 0 2 5}$ & 0,413 & $\mathbf{0 , 0 2}$ \\
\hline Nachbarn vs Schall MW & 0,36 & $\mathbf{0 , 0 3 8}$ & 0,336 & 0,05 \\
Ant ICU vs Alter & 0,136 & 0,259 & 0,094 & 0,3265 \\
Ant ICU vs SAPSII-Score & $-0,095$ & 0,326 & $-0,205$ & 0,1625 \\
Ant ICU vs EPS-Score & 0,3 & 0,0725 & 0,333 & 0,0515 \\
\hline Ant ICU vs Ramsay-Score & 0,356 & $\mathbf{0 , 0 4}$ & 0,28 & 0,0875 \\
\hline
\end{tabular}

Eingerahmter Wert der Korrelation ist auf dem Niveau $\mathrm{p} \leq 0,05$ signifikant.

Ant ICU: Anteil der ICU-Arousalindices

\subsection{Statistik}

Die Tabelle 3.7 zeigt die statistische Berechnung der Korrelationen nach Pearson und SpearmansRho. Nur signifikante Korrelationen werden graphisch dargestellt.

Es bestand eine statistisch signifikante Korrelation zwischen dem Anteil des ICU-Arousalindex und der totalen Schlafzeit $(\mathrm{p}<0,05)$, als auch zum Schlafeffizienzindex (SEI) $(\mathrm{p}<0,05)$. Eine statistisch relevante Korrelation ergab sich auch bei dem Anteil des ICU-Arousalindex zur Anzahl der Nachbarn $(\mathrm{p}<0,05)$ und der Anzahl der Nachbarn zum Schallmittelwert $(\mathrm{p}<0,05)$. Auch die Korrelation zwischen dem Anteil des ICU-Arousalindex und dem Ramsay-Score $(\mathrm{p}<0,05)$ stellte sich als signifikant heraus. Hier muss jedoch beachtet werden, dass nur der Ramsayscore 1 und 2 vergeben wurde. Für diesen Fall erübrigt sich eine statistische Aussage. Keine statistische Signifikanz ergab sich für den Anteil der ICU-Arousalindices zu Schallmittelwert, Schallmaximum, Alter, SAPS II- und EPS-Score, sowie AWI zu Schallmaximum. Die korrelierenden Paare sind in den Grafiken 3.10 bis 3.12 zusammengefasst. In die Streudiagramme wurden Regressionsgeraden einbezogen. 


\section{Abbildung 3.10: Anteil der ICU-Arousals versus TST und Ramsay}

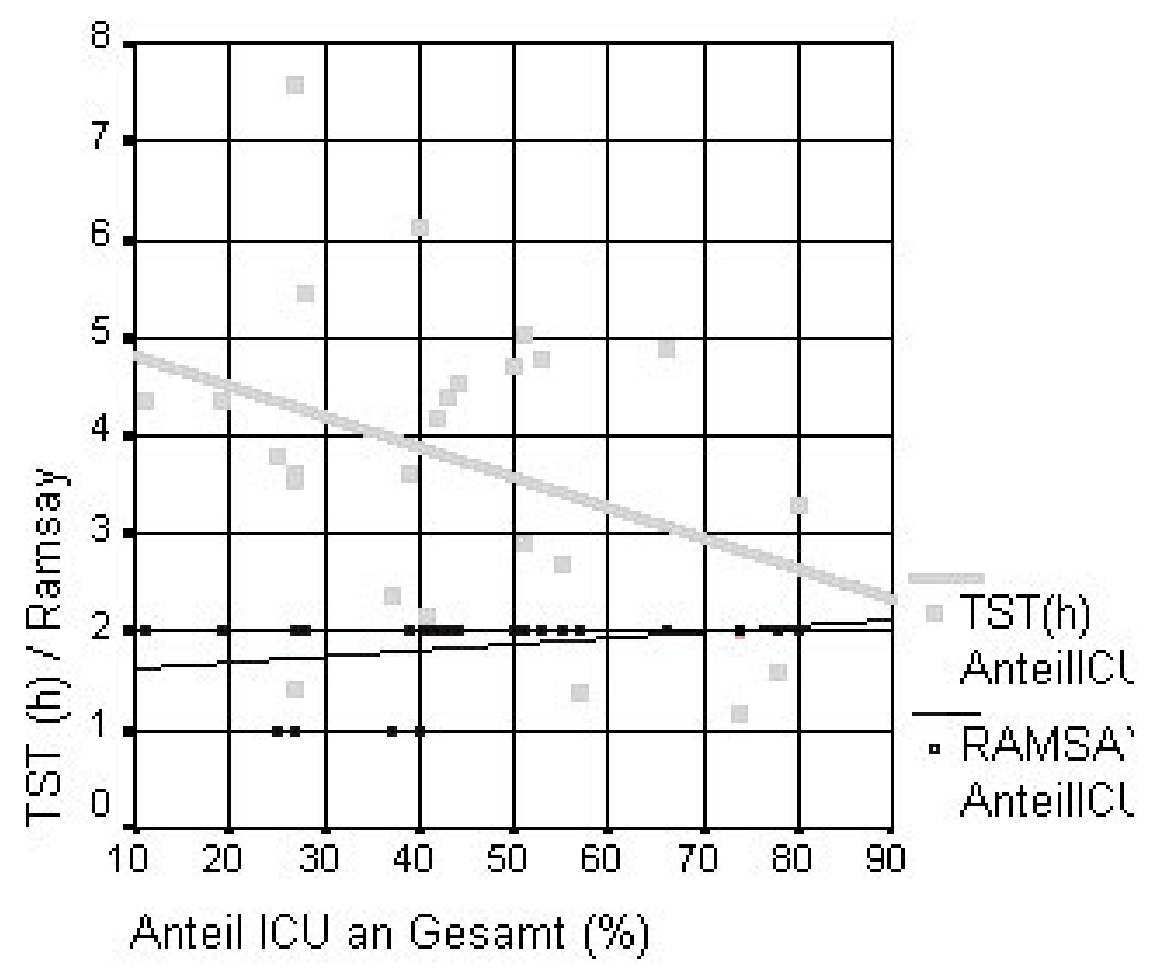

In dieser Abbildung ist einerseits die totale Schlafzeit (TST) und andererseits der Ramsay-Score gegen den Anteil der ICU-Arousals an den Gesamt-Arousals aufgetragen. Man erkennt, dass Patienten mit viel Schlaf einen geringeren Anteil an ICU-Arousals aufwiesen. Je besser also der Schlaf, desto geringer ist die Anzahl der ICU-Arousals. Eine statistische Aussage über den Ramsay-Score erübrigt sich in dieser Untersuchung. 
Abbildung 3.11: Anteil der ICU-Arousals versus SEI

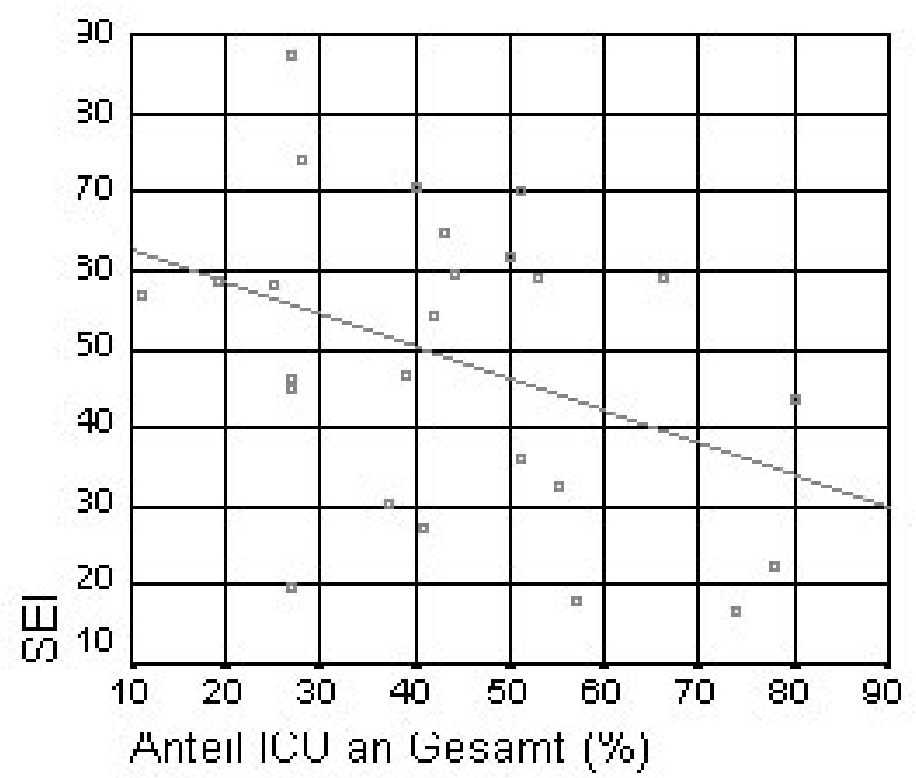

Diese Abbildung zeigt, dass Patienten mit hoher Schlafeffizienz auch einen geringeren Anteil an ICU-Arousals aufweisen. Ein geringer SEI geht allerdings mit einem hohen Anteil an ICU-Arousals einher.

In Abbildung 3.10 ist einerseits die totale Schlafzeit (TST) und andererseits der RamsayScore gegen den Anteil der ICU-Arousals an den Gesamt-Arousals aufgetragen. Man erkennt, dass Patienten mit viel Schlaf einen geringeren Anteil an ICU-Arousals aufwiesen. Je besser also der Schlaf, desto geringer ist die Anzahl der ICU-Arousals.

Die Abbildung 3.11 zeigt, dass Patienten mit hoher Schlafeffizienz auch einen geringeren Anteil an ICU-Arousals aufweisen. Ein geringer SEI geht allerdings mit einem hohen Anteil an ICU-Arousals einher.

In Abbildung 3.12 (S. 59) ist die Anzahl der Nachbarpatienten in Relation zum Lärmmittelwert und zum Anteil der ICU-Arousals gesetzt worden. Deutlich wird, dass Patienten, die nur einen oder gar keinen Nachbarn hatten, durchschnittlich von weniger Lärm umgeben waren und weniger durch ICU-Arousals beeinträchtigt waren, als Patienten mit 2 oder mehr Bettnachbarn. Dies bedeutet, dass Patienten mit 2 oder mehr Nachbarn häufiger ICU-Arousals aufweisen, als Patienten in einem Einzelzimmer, bzw. ohne Bettnachbar. 
Abbildung 3.12: Nachbarpatient vs Schallmittelwert und Anteil der ICUArousals an Gesamt

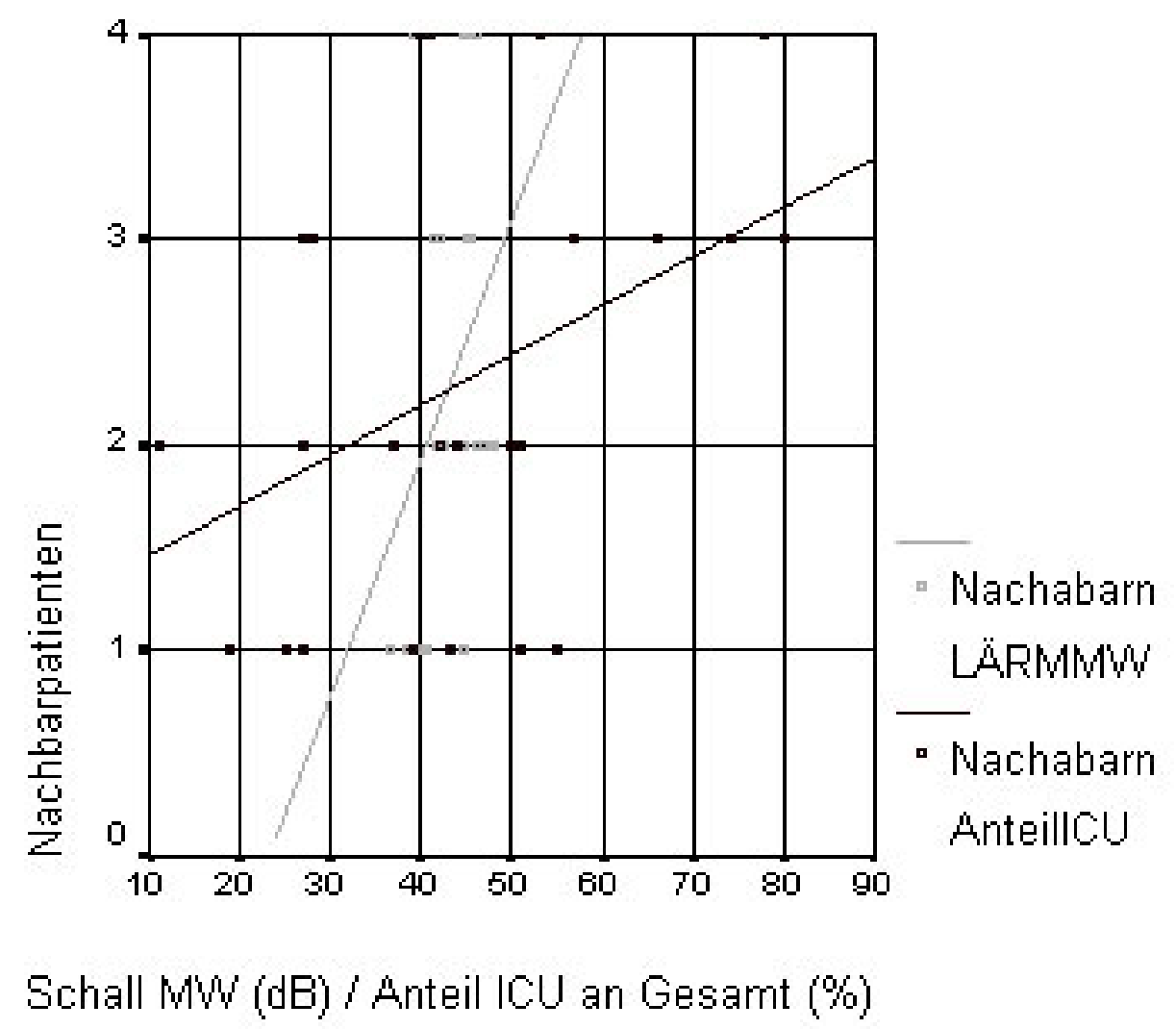

Hier ist die Anzahl der Nachbarpatienten in Relation zum Lärmmittelwert und zum Anteil der ICU-Arousals gesetzt worden. Deutlich wird, dass Patienten, die nur einen oder gar keinen Nachbarn hatten, durchschnittlich von weniger Lärm umgeben waren und weniger durch ICU-Arousals beeinträchtigt waren, als Patienten mit 2 oder mehr Bettnachbarn. Dies bedeutet, dass Patienten mit 2 oder mehr Nachbarn häufiger ICUArousals aufweisen, als Patienten in einem Einzelzimmer, bzw. ohne Bettnachbar. 


\section{Kapitel 4}

\section{Diskussion}

\subsection{Studienort und Studienplanung}

Die Untersuchung fand im Zeitraum Dezember 2000 bis September 2001 auf den Intensivstationen 0112 und 0113 im Klinikum Göttingen statt. Die Patienten waren den Einflüssen und Störungen eines normalen Ablaufs einer Intensivstation ausgesetzt. Die Untersuchung erfolgte unter Realbedingungen. Daraus ergaben sich nicht nur zusätzliche Fehlerquellen, sondern auch Probleme, die Organisation und Durchführung betrafen.

Zunächst ist der beachtliche Platzbedarf für die Messinstrumente (Siehe Kapitel 2.4.1 S.21) und Untersucher zu nennen. Die Tätigkeit von Schwestern und Ärzten durfte dadurch nicht behindert werden. Vorausgesetzt wurde, dass die Patienten der Untersuchung den üblichen Pflege- und Behandlungsabläufen unterzogen wurden. Nicht immer konnte das eingehalten werden, sodass ein Kompromiss zwischen Beeinträchtigung der Patientenversorgung, zumutbaren Arbeitsbedingungen für Ärzte und Schwestern, und einer erfolgversprechenden Untersuchungsdurchführung gefunden werden musste. Auf den Stationen war es üblich, dass die Patienten während des Nachtdienstes, von 20 bis 6 Uhr, gewaschen und die Betten frisch bezogen wurden. Um die pflegerischen Maßnahmen rechtzeitig vor 6 Uhr beenden zu können, war es nötig, bereits um 4 Uhr mit Waschen und Betten zu beginnen. Einen Patienten zu waschen, der unter PSG-Kontrolle ist, war aufgrund der vielen Kabel, der sensiblen und aufwendigen Technik nicht realisierbar und nicht sinnvoll. Deshalb einigte man sich mit den Schwestern darauf, die Probanden nicht innerhalb des Messzeitraumes zu waschen. Dies war 
der einzige Kompromiss, der eingegangen werden musste, wenn auch die Daten dadurch im positiven Sinne verfälscht wurden. Während die Patienten, die nicht an der Studie teilnahmen, in der Zeit von 4-6 Uhr geweckt und ca 15-30 Minuten versorgt wurden, hatten unsere Probanden die Möglichkeit in dieser Zeit zu schlafen.

\subsection{Population}

Die untersuchte Population war heterogen. Das Alter der Patienten reichte von 20 bis 76 Jahren, woraus sich schon Schwierigkeiten des Vergleichs ergaben, da Menschen in verschiedenen Altersabschnitten unterschiedliche Schlafgewohnheiten aufzeigen. Die Häufigkeit der Arousals ist altersabhängig (siehe Steinberg et al. 2000 S.146). Aus diesem Grund hat Steinberg eine Altersaufteilung von 10-19, 20-39, 40-59 und älter als 60 Jahre vorgeschlagen (siehe Tab. 4.1 S.66). Für alle anderen Schlafparameter (TST, SPT, SOL etc.) gelten nach Carskadon und Dement (2000) sowie Steinberg et al. (2000) einheitliche Werte, die nicht oder nur in geringem Maße vom Alter abhängig sind. Da ausschließlich Patienten zwischen 20 und 76 Jahren untersucht wurden, können hier keine Aussagen über Jugendliche (bis 19 Jahre), Kinder, Kleinkinder und Säuglinge getroffen werden.

\subsubsection{Auswahl}

Die Auswahl der Patienten ist nicht randomisiert, sondern auf eine bestimmte Gruppe bezogen, die keine vorbestehenden Schlafstörungen aufwiesen. Die Auswahl erfolgte streng nach den in Kapitel 2.3.2 (S.19) und 2.3.3 (S.20) dargelegten Aufnahme- und Ausschlusskriterien, da viele Erkrankungen mit einem pathologischen EEG einhergehen können.

Wir haben versucht, die Faktoren die den Schlaf beeinträchtigen auf das Umfeld der Intensivstation und Krankheit der Patienten einzuschränken. Septische und infektiöse Patienten wurden aus Sicherheitsgründen von der Untersuchung ausgeschlossen. Patienten, bei denen eine Akutintervention nötig gewesen wäre, hat es in der Studie nicht gegeben. 


\subsection{Untersuchungsverfahren}

\subsubsection{Polysomnographie (PSG)}

Die PSG ist eine langjährig etablierte Messmethode, die Schlaf qualitativ und quantitativ messen und erfassen kann, wie z.B. folgende Autoren in ihren Untersuchungen zeigten: Calvete Vazquez et al. (2000), Carskadon und Dement (2000), Ferrara et al. (1999), Freedman et al. (1999 und 2001), McGuire et al. (2000), Rechtschaffen und Kales (1968), Rosenberg et al. (1995), Schwab (1994), Steinberg et al. (2000) und Topf et al. (1995).

In unserer Untersuchung ergaben die Auswertungen der gemessenen Nächte mit PSG sinnvolle und aussagekräftige Daten, die mit denen der Literatur vergleichbar sind (z.B. Aaron et al. (1996), Aurell und Elmqvist (1985), Calvete Vazquez et al. (2000), Carskadon und Dement (2000), Ferrara et al. (1999), Freedman et al. (2001), Hansell (1984), Helton et al. (1980), McGuire et al. (2000), Rechtschaffen und Kales (1968), Rosenberg et al. (1995), Schwab (1994), Steinberg et al. (2000), Topf et al.(1995), Walder et al. (2000), Woodward et al. (2000)). Auch Freedman et al. untersuchten 2001 intensivpflichtige Patienten mittels PSG und Soundlevelmeter.

Um die PSG jedoch auf einer Intensivstation standardisieren zu können, ist ein hoher technischer und personeller Aufwand nötig. Zwar kann die PSG auch auf einer Intensivstation zu Schlafuntersuchungen verwendet werden, für die Routine ist das Verfahren wegen des hohen Aufwandes jedoch nicht geeignet. Zur Diagnose von Schlafstörungen und deren Erforschung ist die PSG nach wie vor Goldstandard.

\subsubsection{Soundlevelmeter}

Das verwendete mobile Gerät war batteriebetrieben; es konnten aufgrund ungenügender Schallableitung einige Messungen nicht verwendet werden. Eine batterieunabhängige Messung wäre effektiver gewesen und hätte zu weniger Datenverlust geführt. Das Soundlevelmeter wurde einen Meter entfernt vom Patienten platziert. Bewegungen des Patienten (z.B Links- o. Rechtsseitenlage) führten zu Abweichungen. Der Schallmeter gibt den Lärmpegel im Raum und in der näheren Umgebung des Patienten wieder. Die PSG gekoppelt mit Soundlevelmeter ist ein etabliertes und standardisiertes Verfahren in der somnologischen Forschung, 
das auch in unserer Untersuchung zu aussagekräftigen Ergebnissen führte (Aaron et al. 1996, Freedman et al. 2001, Steinberg et al. 2000).

\subsubsection{Online Registrierung mit Computer}

Die online Registrierung mit Computer, die Teil der Somnologie mit PSG ist, erwies sich als sehr wertvoll. Erstens wurden alle Daten und Signale in Echtzeit aufgenommen. Zweitens konnte eine große Datenmenge übersichtlich gespeichert und danach verarbeitet werden. Software gestützt war es möglich, schnell Schlafparameter zu errechnen und Hypnogramme zu erstellen, um somit Qualität und Quantität des Schlafes der Patienten beurteilen zu können. Eine gekoppelte Videoüberwachung mit Computer könnte für die weitere Auswertung von Nutzen sein. Per Video hätten Arousals oder Lageänderungen des Patienten oder die Herkunft ungeklärter Schallspitzen eindeutiger geklärt werden können. Aktionen der Schwestern

oder Ärzte, die beispielsweise ein Arousal auslösten und dem Untersucher entgangen waren, hätten nachträglich dokumentiert werden können.

\subsubsection{Akzeptanz}

Die Akzeptanz der Untersuchung mit PSG betrifft einerseits Patienten und deren Angehörige, andererseits das Pflegepersonal und Ärzte. Nur vier Patienten brachen die Studie vorzeitig ab. Auch die Angehörigen, sofern sie anwesend waren, unterstützten die Untersuchung. Sinn und Zweck der risikolosen Studie, nämlich den Ursachen der Schlafstörungen auf einer Intensivstation auf den Grund zu gehen, war sowohl Angehörigen als auch Patienten einleuchtend. Die Tätigkeit des Pflegepersonals wurde von der Studie kaum beeinflusst. Da die Ärzte nachts nur im Notfall Maßnahmen ergreifen mussten, sind sie nur selten mit der Untersuchung konfrontiert worden. Tätigkeiten wie das Legen peripher venöser oder arterieller Zugänge waren auch während der laufenden Untersuchung möglich. Folglich wurde die Untersuchung retrospektiv von Seiten der Ärzte und des Pflegepersonals positiv beurteilt und unterstützt. 


\subsection{Auswertung}

Die Auswertung der PSG erfolgte nach den Regeln von Rechtschaffen und Kales (1968) und der DGSM (Penzel et al. 1993). Mit der erweiterten Auswertung der Arousals nach den Kriterien der ASDA (1997) (Kapitel 2.6.4 S.29) konnten die Arousals speziell für diese Untersuchung analysiert werden.

\subsubsection{Die ICU-Arousals}

Da spezielle Bedingungen auf einer ICU herrschen und unterschiedlichste Lärmqualitäten, wie Monitor-, Perfusoralarme und andere typische Geräusche und deren Auswirkungen erfasst werden sollten, mussten Arousalklassen neu definiert werden. Die bereits vorhandenen Standard-Arousals wurden somit um die ICU-Arousals erweitert. In diesen wurde den Schallquellen ein entsprechendes Arousal zugeordnet. Dabei wurden nur die wichtigsten berücksichtigt, alle anderen fasste man unter der Kategorie "Schall undefiniert" zusammen. Somit resultierten die ICU-Arousals: Schall undefiniert, Monitor, Personal, Nachbarpatient, RR, Perfusor, Telefon, Beatmung, und Intervention. Durch den Vergleich mit den Daten von Carskadon und Dement (2000) sowie Steinberg et al. (2000) konnte dargelegt werden, dass mit der Einführung der ICU-Arousals die Anzahl der Standard-Arousal nicht beeinflusst wurde. (Siehe Kap. 4.5.3 S.68).

\subsection{Ergebnisse}

\subsubsection{Schlafparameter}

Bei den 25 Patienten wurde eine normale Zeit im Bett (TIB), im Mittel 7,67 Stunden, gemessen. Normal verbringt ein Mensch im Mittel 5 bis 9 Stunden im Bett. Somit wurde den Patienten genügend Zeit gegeben zu schlafen. Diese wurde auch zum Schlafen genutzt, die Schlafperiodendauer (SPT) betrug 6,66 Stunden, das heisst, dass die Patienten 86,8\% der Zeit zum Schlafen nutzen konnten. Jedoch wurden davon im Mittel nur 3,76 Stunden (TST) geschlafen. Das entspricht einem Schlafeffizienzindex (SEI) von 49\%, der deutlich niedriger liegt als die von Steinberg et al. (2000) ermittelte Norm der Schlafeffizienz von über 90\%. 
Bei unserer Gruppe von Intensivpatienten ist im Vergleich zum Schlaf normaler Probanden eine Verminderung des Schlafeffizienzindex (SEI) und ein Anstieg der Einschlaflatenz (SOL) festzustellen. Die Zeit des Wachstadiums (W) nimmt deutlich zu, die Stadien S1 und S2 sind ebenfalls auf Kosten des $\delta$ - und REM-Schlafs vermehrt. Dies entspricht den Ergebnissen von Aurell und Elmqvist (1985), Johns et al. (1974), Rosenberg et al. (1995), Schwab (1994) und Sebilia (1981). Der für die Erholung und Genesung so wichtige REM-Schlaf ist von vergleichsweise 20\% - 25\% (nach Steinberg et al. 2000) auf weniger als 2\% im Mittel gesunken. Tab. 4.1 (S. 66) enthält orientierende Kennwerte der Schlafparameter wie Steinberg et al. (2000) sie zusammengefasst haben. Einen direkten Vergleich bietet Abbildung 4.1 (S. 70).

Alle genannten Autoren stimmen darin über ein, dass auf einer Intensivstation der Schlaf tiefgreifend von der Umgebung beeinflusst wird. Die Schlafeffizienz von $49 \%$ ist alarmierend, da die Patienten weniger als die Hälfte der Zeit schliefen.

\subsubsection{Geräuschpegel}

Der Geräuschpegel auf dieser Intensivstation lag im Schnitt bei 42,94 dB $\pm 3,08 \mathrm{~dB}$ (36,60 dB; 47,80 dB). Zum Vergleich herrschen nach Walder et al. (2000) in einem Schlafzimmer stets weniger als $40 \mathrm{~dB}$, in einem ruhigen Büro weniger als $50 \mathrm{~dB}$ und in einer Disco herrschen ca. 120 dB Lärmbelastung. Bei einigen der Messungen war es also im Mittel so laut wie in einem Büro. Lediglich die Mittelwerte der minimalen Schallwerte liegen mit 36,56 dB $\pm 3,91 \mathrm{~dB}$ gerade noch unterhalb derjenigen, die für ein Schlafzimmer gelten. Jedoch kann die von Bentley et al. (1977) geforderte Maximalgrenze nicht eingehalten werden. Bentley et al. fordern für einen erholsamen Schlaf weniger als $30 \mathrm{~dB}$. Diese Voraussetzungen konnten auch in unserer Studie nicht erfüllt werden. Vielmehr überschritten die Maximalwerte die Grenzen bei Weitem. Mit 85,45 $\pm 12,64 \mathrm{~dB}$ glichen sie eher einer Disco als einem Schlafzimmer. Ein Proband hatte die stärkste Lärmbelastung mit 95,50 dB. Eine differenzierte Aufteilung des Schalls soll im folgenden Kapitel behandelt werden.

\subsubsection{Schall- und Arousalprofile}

Auf der Intensivstation wurde folgender Arbeitsrhythmus beobachtet:

Ab 21 Uhr wurden die Patienten für die Nacht versorgt, es erfolgte die Informationsweiter- 
Tabelle 4.1: Orientierende Kennwerte nach Steinberg et al. (2000)

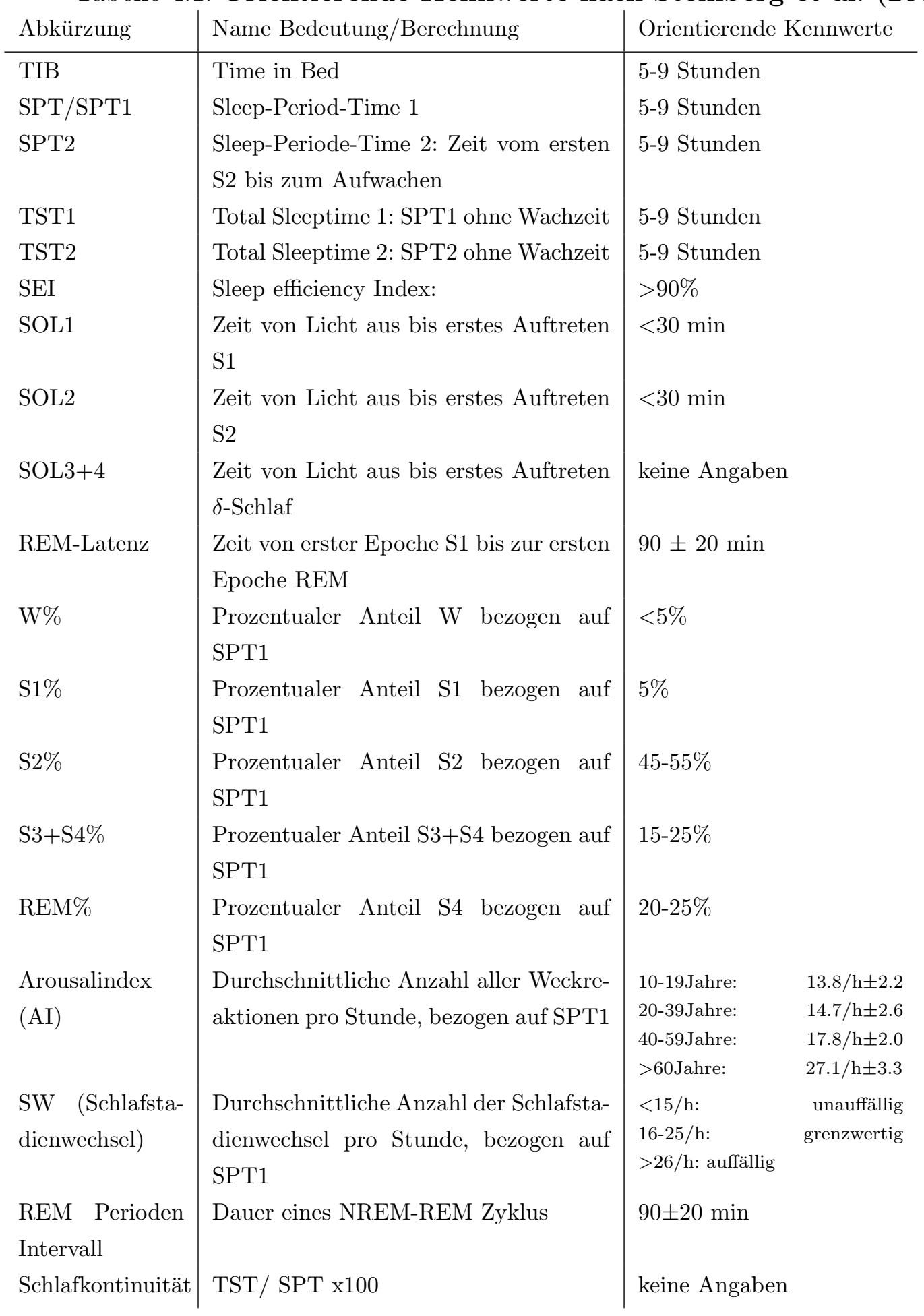

Orientierende Kennwerte aus Steinberg et al. (2000) S.146; Sie unterschieden zusätzlich Respiratorischer Arousalindex (RAI), PLMS-Arousalindex (PLMS-AI) (periodic leg movement associated) und endogener Arousalindex (EAI) 
gabe an die neue Schicht der Pflege und eine Visite der Ärzte. Gegen 22 Uhr wurde die nächtliche Medikation verabreicht. Ab ca 24 Uhr gingen die Ärzte und Schwestern nur noch Alarmen oder Notfällen nach. Es trat eine Ruhephase ein, die ungefähr bis 4 Uhr andauerte. Dann nahmen Aktivität und damit Unruhe auf der Station wieder zu. Die ersten Patienten wurden gewaschen, damit alle Patienten bis 6 Uhr versorgt waren. Dieser Rhythmus konnte in den Schall- und Arousalprofilen wiedergefunden werden. Aus Kapitel 3.2.4 (S.52), geht hervor, dass Lärm zu den Zeiten 'Eins' und 'Vier' vorherrschend war, aber 'Zwei' und 'Drei' ruhigere Zeiten zu sein schienen. Die Zeiten 'Zwei' und 'Drei' werden fortan als Ruhezeiten und die Zeitpunkte 'Eins' und 'Vier' als Aktivitätszeiten bezeichnet, sie sind durch eine besondere Dichte pflegerischer Maßnahmen gekennzeichnet. Die Ruhezeit ist also nur 4 Stunden lang. Dies ist beängstigend, da die benötigte Schlafzeit nach Steinberg et al. (2000) mindestens 5 bis zu 9 Stunden betragen sollte. Sicherlich war es dem Patienten auch möglich, zu den lärmintensiveren Zeiten zu schlafen, jedoch ist der Schlaf dann deutlich verändert, wie die Abbildungen 3.7 (S.53) - 3.9 (S.55) zeigen. Weiter ist durch die kurze Ruhezeit eine circadiane Rhythmik, die laut Hobson (1989) und Sebilia (1981) wichtig für Genesung und Gesundheit des Organismus ist, nicht vorhanden. Die circadiane Rhythmik beinhaltet z.B. durch Licht beeinflussbare Zyklen und Gewohnheiten des menschlichen Organismus. Dazu gehören insbesondere der ausreichende Nachtschlaf und die Aktivität zur Tageszeit.

Um die Schlafzeiten zu verlängern, könnten die Ruhezeiten verlängert und pflegerische und ärztliche Maßnahmen von der Nacht auf den Tag verlegt werden. Durch eine Verlängerung der Ruhezeit z.B. von 22 Uhr bis 6 Uhr könnte diese verdoppelt werden.

Wie schon erläutert, kann ein Schallpeak ein Arousal verursachen. Abbildung 3.8 (S.54) verdeutlicht das Verhalten der Arousalindices zur Zeit. Insgesamt waren zu Zeiten vermehrten Lärms (1 und 4, Aktivitätszeit) auch vermehrt Arousals zu beobachten. Besonders eindrücklich verhielt sich der prozentuale Anteil des ICU-Arousalindex zur Zeit. Das Verhältnis der Arousals zueinander war zu den Aktivitätszeiten deutlich zu Gunsten der ICU-Arousals verschoben. Das war besonders deutlich im Zeitintervall 'Vier'. Gegensinnig sind also zu den Ruhezeiten weniger ICU-Arousals als zu den Aktivitätszeiten zu beobachten. Das Verhältnis der Arousals zieht eine verminderte Schlafzeit (TST) zu den Aktivitätszeiten, aber eine Steigerung der TST zu den Ruhezeiten nach sich. Analog hierzu ist das Verhalten des Auf- 
wachindex. Der Patient konnte also zu den Ruhezeiten deutlich besser schlafen, wies weniger ICU-Arousals auf und erwachte auch weniger häufig, als zu den Aktivitätszeiten 'Eins' und 'Vier'. In den Ruhephasen wachen die Patienten seltener auf als in den Aktivitätsphasen. Es war also ein starker Einfluss von Aktivität und Arbeitsabläufen auf den Schlaf der Patienten festzustellen. Wir fanden eine Verminderung des Schlafs (TST) zu Zeiten erhöhter Aktivität und erhöhten Schalls, auch der Schlaf wurde zu diesen Zeiten häufiger durch Arousals und Wachphasen unterbrochen, als zu den Ruhezeiten. Walder et al. (2000) gelang es in ihren Untersuchungen durch Veränderung und Reglementierung der Arbeits- Stationsabläufe die Ruhezeiten von 23:00 bis 5:00, also auf 6 Stunden zu verlängern. Sie konnten eine signifikante Verringerung der Störintensität und des mittleren Schallpeaks feststellen. Jedoch konnten sie nicht nachweisen, dass Hintergrundgeräusche verringert wurden.

Die Regelungen von Walder et al. 2000 (S.2243):

- Alle Türen wurden systematisch geschlossen gehalten.

- Monitoralarme wurden maximal reduziert.

- Die Pflege wurde von 23:00 bis 5:00 Uhr auf das Nötigste reduziert.

- Unterhaltung war nur mit leiser Stimme erlaubt; Telefone, Piepser, Radio oder Fernsehen waren untersagt.

- Es durften kein direktes Licht oder Taschenlampen benutzt werden.

Durch Veränderungen der Arbeitsabläufe und des Umfeldes sowie des Verhaltens des Pflegepersonals und der Ärzte könnte also eine Reduzierung des Lärms erreicht werden. Dadurch könnten die ICU-Arousals, der AWI und die Wachphasen reduziert, sowie die gesamte Schlafzeit (TST) verlängert werden.

\subsubsection{Vergleich mit den Ergebnissen Carskadon und Dement (2000) sowie Steinberg et al. (2000)}

Carskadon und Dement (2000) sowie Steinberg et al. (2000) untersuchten den Schlaf gesunder Probanden. Unsere Ergebnisse werden im Folgenden mit den oben genannten Arbeiten verglichen. Man muss beachten, dass deren Ergebnisse an normalen und nicht kranken oder 
intensivpflichtigen Probanden unter weitgehend normalen Bedingungen erhoben wurden. Der Vergleich mit einem Normkollektiv dient dazu, festzustellen, ob und wie sich die Schlafparameter und die Schlafarchitektur von Patienten einer Intensivstation vom Normkollektiv unterscheiden (Siehe Abb. 4.1 S.70).

Hierbei fällt auf, dass im Durchschnitt die Patienten unserer Untersuchung eine längere Einschlaflatenz aufweisen. Vergleichsweise sollte ein Gesunder nicht mehr als 30 Minuten zum Einschlafen benötigen, die Patienten unserer Untersuchung benötigten jedoch mehr als 35 Minuten. Die Zeit im Bett, die Schlafperiode und die Totale Schlafzeit liegen im Normalfall bei 5-9 Stunden. Unsere Patienten schliefen im Mittel nur 3,76 Stunden, bei einer Schlafperiode von 6,66 Stunden. Die Zeit im Bett, bei unserer Untersuchung der Dauer der Messung entsprechend, betrug durchschnittlich 7,67 Stunden. Von der Zeit, die Patienten im Bett polysomnographisch überwacht wurden, waren sie zu 44,67\%, also mehr als die Hälfte der Zeit wach, die Stadien des Schlafes S1 und S2 nahmen 25,7\% respektive 21,65\% der geschlafenen Zeit ein. Die wichtigen und erholsamen $\delta$ - und REM-Schlafstadien betrugen gemeinsam im Mittel 7,98\%, sie sollten aber nach Carskadon und Dement (2000) und Steinberg et al. (2000) jeweils $15 \%$ bis $25 \%$ betragen. Die Altersgruppe der 20- bis 39- und der 40- bis 59-Jährigen verzeichnet in unserer Untersuchung einen Anstieg der Arousals auf durchschnittliche 25,27 respektive 22,59 Arousals/h. Vergleichsweise niedrig ist die Zahl der Arousal nach Carskadon und Dement (2000) und Steinberg et al. (2000), die für die beiden Altersgruppen 13,8 und 14,7 Arousals/h im Mittel als 'Norm' betrachten. Bei der Altersgruppe der über 60-Jährigen sind Arousals bis zu 27,1/h im Toleranzbereich. Vergleichend mit unserer Untersuchung wies die gleiche Altersgruppe nur 22,67 Arousals/h auf.

Zusammenfassend fällt auf, dass die Schlafeffizienz (SEI) zusammen mit der totalen Schlafzeit (TST) erheblich sank, der Wachanteil entsprechend auf knapp die Hälfte der zu schlafenden Zeit anstieg. Die Latenzzeit bis zum Einschlafen verlängert sich deutlich. In der Schlafperiode (SPT) sind die Stadien W, S1 und S2 vorherrschend; Wach und Stadium 1 sind alarmierend auf Kosten des $\delta$ - und REM-Schlafes verlängert. Verschwindend gering oder oft fehlend sind der $\delta$ - und der REM-Schlaf, die laut Carola et al. (1990) für die Erholung so wichtig sind. Vermehrte Arousals von bis zu 23 pro Stunde, gepaart mit einem Aufwachindex von 4,42 bedeutet, dass die Patienten in einer Stunde mehr als 27 mal eine Weckreaktion aufweisen 
Abbildung 4.1: Graphische Gegenüberstellung der Normwerte von Carskadon und Dement (2000) sowie Steinberg et al. (2000)

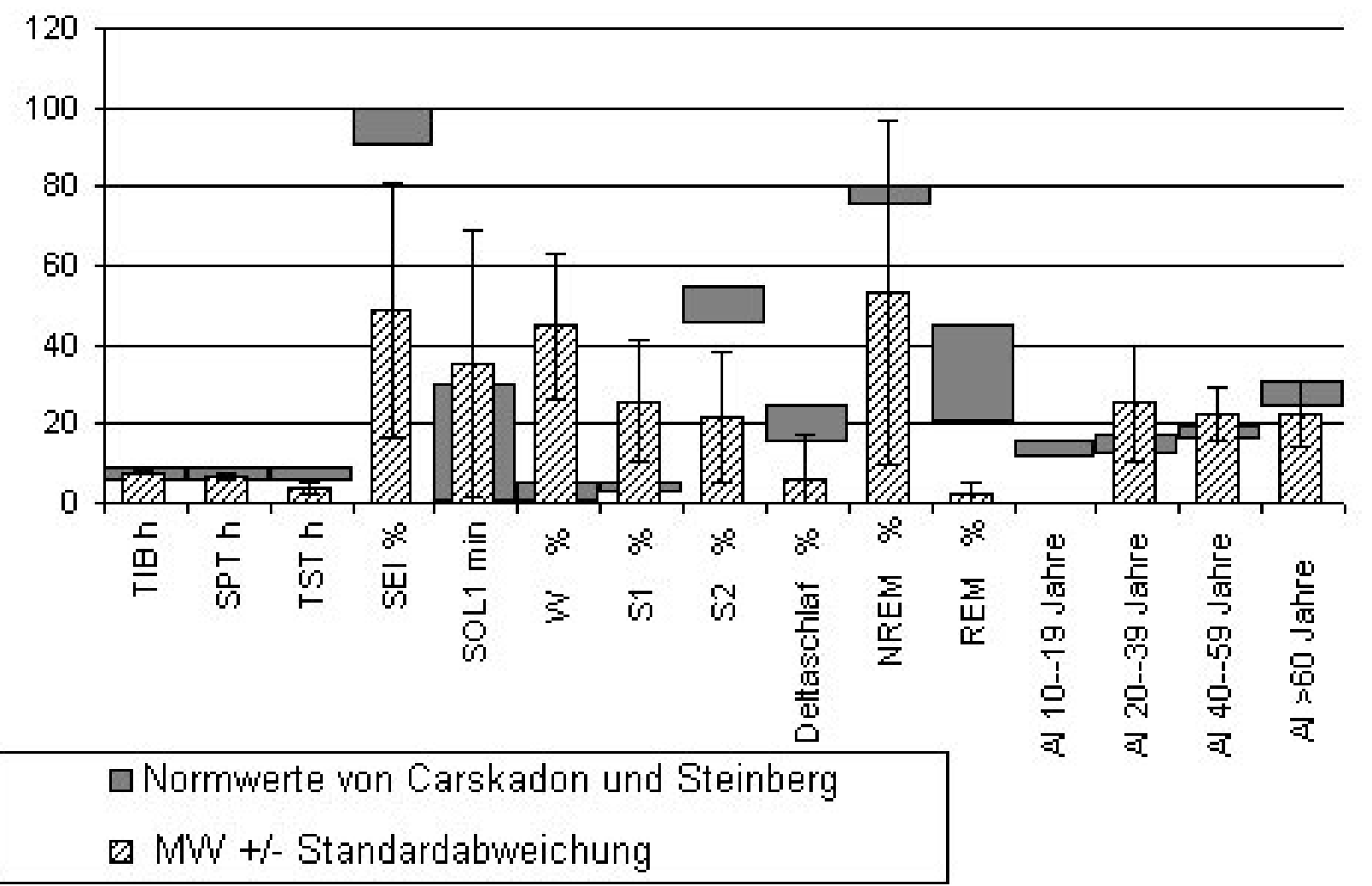

Die Normwerte nach Carskadon und Dement (2000) und Steinberg et al. (2000) sind als graue Balken dargestellt. Die hellen Säulen entsprechen den Werten unserer Untersuchung mit der jeweiligen Standardabweichung. 


\section{Abbildung 4.2: Hypnogramm eines Gesunden im Vergleich mit pathologi-} schem Hypnogramm

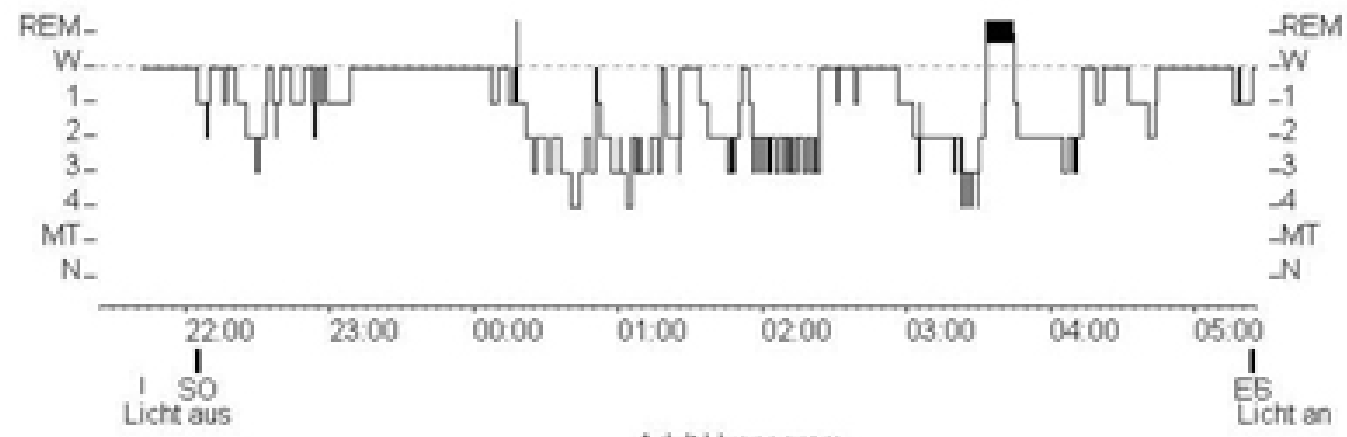

Adult Hypnogram

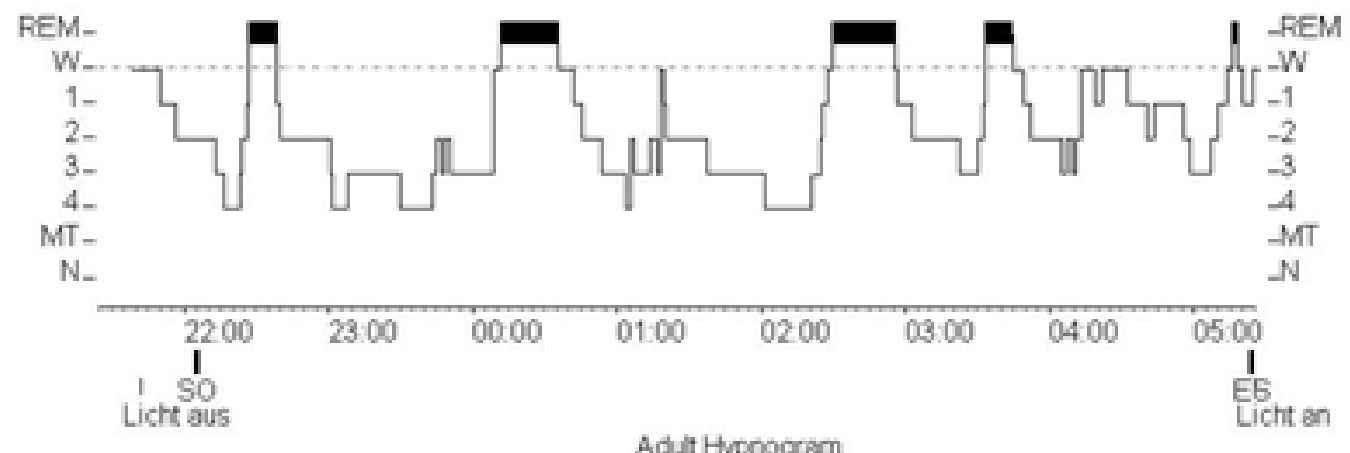

Das obere Hypnogramm zeigt eine pathologische Schlafarchitektur eines unserer Patienten. Das Untere zeigt die Schlafarchitektur eines gesunden Menschen.

SO: Sleep-Onset - Schlafbeginn (erstes Auftreten S1 nach "Lights Off")

ES: End-of-Sleep - Schlafende (letztes Schlafstadium vor "Lights On")

oder gänzlich aufwachen. Wobei jüngere Patienten mehr Arousals aufweisen als ältere.

Diese Veränderungen der Schlafparameter und die häufigen Störungen sind Besorgnis erregend und führen letztlich zu den beobachteten Schlafdefiziten bei den untersuchten Patienten. Ein beispielhaftes fragmentiertes Hypnogramm ist in Abbildung 4.2 (S.71) zu sehen.

Abbildung 4.2 zeigt, dass der Schlaf auf die Stadien W, S1 und S2 reduziert ist. Wachepisoden und 'leichter' Schlaf dominieren. Regelmäßigkeiten oder Schlafzyklen sind nicht zu erkennen. REM und $\delta$-Schlaf sind nur spärlich vorhanden. Arousals sind in diesem Hypnogramm nicht dargestellt. 


\subsubsection{Arousals}

Von den Gesamt-Arousals sind 55,9\% Standard-Arousal und 44,1\% sind ICU-Arousals, bedingt durch den Aufenthalt auf einer Intensivstation und der damit verbundenen Überwachung und Pflege (Siehe Abb. 3.5 S.49). Wir stellten fest, dass ein Patient der Intensivstation, durch Einflüsse dieser Umgebung nahezu doppelt so viele Arousals aufweist wie in dem von Carskadon und Dement (2000) sowie Steinberg et al. (2000) beschriebenen Normkollektiv (Siehe Tab. 4.1 S.66). Ähnliches beobachtet man bei den akustischen und nicht-akustischen Arousals in Abbildung 3.5 (S.49). 42,2\% der Arousals sind akustisch, also durch Lärm verursacht. Mit einer Reduktion der akustischen Einflüsse könnte man also eine Arousalreduktion um mehr als 40\% erreichen! Es ist festzustellen, dass in dieser Untersuchung nicht eine hauptverantwortliche Geräuschquelle eruiert werden konnte, sondern es ist scheinbar die Gesamtheit aller Geräuschquellen, die den Pegel erhöhen. Am häufigsten sind die undefinierten Arousals. Sie sind durch einen unbekannt Schallpeak, auch z.B. durch Personal, Monitor, Perfusor, oder Nachbarpatient, verursacht worden. Als zweites ist das Monitor-Arousal zu nennen. Es folgen Arousals verursacht durch das Personal, den Nachbarn, die Blutdruckmessung, Perfusoralarme, das Telefon, Beatmung und Beatmungsalarme sowie durch Interventionen am Patienten. Die Monitor-Arousals, aber auch die durch Nachbarn und Gerätealarme bedingten Arousals könnten durch die in Kapitel 4.5.2 (S.68) genannten Regelungen vermindert werden, um dem Patienten mehr Schlaf und Erholung zukommen zu lassen, wie Olson et al. (2001), Topf (1992) Redeker (2000) und Walder et al. (2000) bestätigt haben. Außerdem könnte zentrales Monitoring und frühzeitiges Quittieren von Alarmen die Monitor- und Perfusor-Arousals reduzieren.

Die Überwachung der Vitalparameter ist wichtig für den Patienten, doch wie schon Calvete Vazquez et al. (2000) feststellten, ist der Gerätealarm ein häufiger Störfaktor. Individuelle Alarmgrenzen des Patientenmonitors könnten helfen, diese Arousals zu verringern. Würden Monitor- und Perfusoralarme im Zimmer des Patienten optisch, in der Zentrale jedoch auch akustisch registriert, ließen sich die Störungen durch Gerätealarme deutlich reduzieren. Da die PSG auf der Intensivstation nicht standardisiert ist, könnten auch die Pflegekräfte den Schlaf der Patienten beurteilen. Edwards und Schuring (1993) erkannten in einer Studie, dass die Schwestern auf einer Intensivstation in $80 \%$ der Fälle richtig einschätzen können, ob sich 
der Patient in einer Wach- oder Schlafphase befindet. Schlaflosigkeit und -defizite könnten somit auch ohne PSG diagnostiziert werden, um den im Schlaf gestörten Patienten durch genannte Reglements zu mehr Ruhe und Erholung, und somit auch zu Schlaf kommen zu lassen.

Zuletzt soll noch auf die Störung durch den Nachbarpatienten eingegangen werden. Leider kann mann nicht immer unterscheiden, ob die Monitor-, Perfusor- und Beatmungsalarme vom Nachbarn oder von den Geräten des Probanden stammen. Alle Geräusche und Störfaktoren durch den Nachbarn könnten beseitigt werden, bestünde eine schalldämpfende Abtrennung der Patienten untereinander, z.B durch Unterbringung in Einzelzimmern. Somit könnte eine weitere Geräuschpegelreduktion und Privatsphäre für den Patienten erreicht werden. Dies würde eventuell einen größeren Platzbedarf bedeuten. Nimmt man auf die hier vorliegenden Platz- und Raumverhältnisse Bezug, müssten sich vor allem die Vierbettzimmer reduzieren. Architektonisch müsste auf dieser Intensivstation sicherlich ein Bettplatz bautechnisch eingebüßt werden, um zusätzliche Einzelzimmer auszustatten.

\subsubsection{Vergleich mit der Studie von Freedman et al. (2001)}

In unserer Studie wurden 9,95 $\pm 5,82$ akustische und 13,61 $\pm 6,52$ nicht-akustische Arousals pro Stunde bei einem Gesamt-Arousalindex von 23,26 $\pm 9,18$ gemessen. Das heißt, dass 42,2\% der gesamten Arousals akustisch bedingt waren.

Freedman et al. (2001) untersuchten 12 Männer und 10 Frauen; mittleres Alter $61 \pm 16$ Jahren $(20$ - 83 Jahre). Der mittlere Aufenthalt auf der Intensivstation vor der Untersuchung betrug $18 \pm 20$ Tage (3 - 80 Tage). Der APACHE-Score, ein Ausdruck für die Morbidität von ICU-Patienten, betrug im Schnitt $57 \pm 28$. Die Patienten wurden über $24 \mathrm{~h}$ beobachtet. Es wurde TST sowie S1, S2, S3, S4 und REM bezogen auf TST, der Schallpegel und der mittlere Arousalindex, unterteilt in akustisch- und nicht-akustisch bedingte Arousals, aufgenommen. Die genannten Parameter werden in Tabelle 4.2 (S.74) denen dieser Untersuchung gegenübergestellt. Die wichtigen Unterschiede liegen darin, dass alle Patienten der Studie von Freedman et al. (2001) intubiert waren und der Aufenthalt vor der Untersuchung auf der Intensivstation mit durchschnittlich 18 Tagen wesentlich länger als 3 Tage bei unserer Untersuchung war. Der Apache Score wurde in unserer Untersuchung nicht erhoben, die Patienten Freedmans et 
Tabelle 4.2: Vergleich mit den Parametern von Freedman et al. (2001)

\begin{tabular}{|c|c|c|c|c|}
\hline & \multicolumn{2}{|c|}{ Freedman et al. } & \multicolumn{2}{|c|}{ Daten d. Untersuchung } \\
\hline & & & & \\
\hline Männer & 12 & & 18 & \\
\hline Frauen & 10 & & 7 & \\
\hline \multirow[t]{2}{*}{ Gesamt } & 22 & & 25 & \\
\hline & MW & Var & MW & Var \\
\hline Alter & 61 & 16 & 52 & 16,01 \\
\hline APACHE & 57 & 28 & - & - \\
\hline \multirow{2}{*}{$\begin{array}{l}\text { Aufenthalt vor Untersuchung (d) } \\
\text { Dauer der Untersuchung (h) }\end{array}$} & 18 & 20 & 2,64 & 2,04 \\
\hline & $24-48$ & & 8 & \\
\hline Patienten intubiert & 22 & & 0 & \\
\hline \multicolumn{5}{|l|}{ Schlafparameter } \\
\hline TST (h) & 8,8 in $24 \mathrm{~h}$ & 5 & 3,8 in $8 \mathrm{~h}$ & 1,18 \\
\hline $\mathrm{S} 1$ (min) & 59 & 33 & 26 & 15 \\
\hline $\mathrm{S} 2(\min )$ & 26 & 28 & 22 & 16 \\
\hline $\mathrm{S} 3 \& \mathrm{~S} 4(\mathrm{~min})$ & 9 & 18 & 6 & 11 \\
\hline REM (min) & 6 & 9 & 2 & 3 \\
\hline Schallpegel & \multirow[b]{3}{*}{59,1} & \multirow[b]{3}{*}{6,1} & \multirow{5}{*}{$\begin{array}{r}\text { Die } \\
\text { wurden } \\
\text { nicht }\end{array}$} & \multirow{5}{*}{$\begin{array}{l}\text { Patienten } \\
\text { tagsüber } \\
\text { gemessen }\end{array}$} \\
\hline Schallpegel Tag $(d B)$ & & & & \\
\hline Mittlerer & & & & \\
\hline Maximum & \multirow{3}{*}{$\begin{array}{l}68,5 \\
85,9\end{array}$} & \multirow{2}{*}{$\begin{array}{l}7,7 \\
5,1\end{array}$} & & \\
\hline Peak & & & & \\
\hline Schallpegel Nacht $(d B)$ & & & & \\
\hline Mittlerer & 56,8 & 4,9 & 42,9 & 3,1 \\
\hline Maximum & 64,6 & 7,5 & 85,5 & 12,6 \\
\hline Peak & 82,8 & 5,3 & & \\
\hline Arousal & & & & \\
\hline Arousalindex (AI) Gesamt (/h) & 11,6 & 5 & 23,3 & 9,2 \\
\hline Schall AI $(/ \mathrm{h})$ & 1,9 & 2,1 & 9,7 & 5,8 \\
\hline Spontaner AI (/h) & 9,6 & 4,9 & 13,6 & 6,5 \\
\hline Schall Arousals (\%) & 11,5 & 11,8 & 42,2 & \\
\hline
\end{tabular}

Die Daten wurden aus Freedman et al. (2001) (S.451-456) entnommen. Die Daten der Population, Schlafparameter, Schallpegel und der Arousals von Freedman und den Daten dieser Untersuchung werden hier gegenübergestellt. 
al. (2001) waren wahrscheinlich schwerer erkrankt, da hier intubierte und beatmete Patienten gemessen wurden. Der mittlere Schallpegel lag bei Freedman et al. (2001) in der Nacht bei 56,8 dB im Gegensatz zu unserer Untersuchung mit 42,9 dB. Das Maximum der Nacht lag mit $64,6 \mathrm{~dB}$ bei Freedman et al. (2001) versus 85,45 wesentlich niedriger als bei unserer Untersuchung. Weiterhin kann man einen Unterschied der Arousalindices erkennen. Der Gesamt-Arousalindex lag bei der Referenzstudie bei 11,6 versus 23,26 Arousals pro Stunde bei uns. Diese Indices teilen sich wiederum in akustische und non-akustische Arousals auf, die sich bei Freedman et al. (2001) auf 1,9 und 9,6 Arousals pro Stunde versus 9,95 und 13,61 Arousals pro Stunde bei unserer Untersuchung beliefen. Somit ergab sich für die akustischen Arousals bei Freedman et al. (2001) ein Anteil von 11,5\%, bei unserer Untersuchung jedoch 42,2\%. Für Freedman et al. (2001) ergab sich der Schluss, dass ein erhöhter Schallpegel bei seiner Untersuchungspopulation nicht die häufigste Ursache für ein Arousal ist, sondern anderweitige z.B. intrinsische Faktoren. Die Patienten der Referenzstudie waren jedoch intubiert und mussten unter Analgosedierung beatmet werden. Die Patienten unserer Untersuchung hingegen waren wach und standen vergleichsweise wenig unter medikamentösem Einfluss. Beide Ergebnisse scheinen sich zu widersprechen, jedoch ist zu beachten, dass ein wacher Patient, der nicht intubiert ist, eine völlig andere Wahrnehmung hat als ein analgosedierter, beatmungspflichtiger Patient. Deshalb ist zu postulieren, dass die akustischen Arousals sehr wohl einen negativen Einfluss auf den Schlaf eines Patienten einer Intensivstation besitzen. Bezogen auf wache, wahrnehmungsfähige Patienten ist der Behauptung von Freedman et al. (2001), akustische Arousals hätten keinen Einfluss auf den Schlaf, zu widersprechen. Über beatmete Patienten kann diese Untersuchung keine Aussage treffen.

Krachman und Criner (2002) stellten fest, dass chronisch respiratorisch insuffiziente Patienten von einer nächtlichen Langzeit-Beatmung mittels Tracheotomie oder NPPV (Noninvasive Positive Pressure Ventilation) profitieren. Auch die Schlafqualität sei unter anderem durch Optimierung des nächtlichen Gasaustausches verbessert worden. Interessant wäre eine Studie, die den Einfluss nächtlicher Langzeit-Beatmung auf die Schlafarchitektur und Arousals darstellt. 


\subsection{Statistik}

Aus der Statistik ergeben sich signifikante Korrelationen, die aufgrund der geringen Fallzahl und der heterogenen Population nur vorsichtig zu gewichten sind. Es werden nur signifikante Korrelationen diskutiert.

\subsubsection{ICU-Arousals}

Nachweislich sinken die Schlafzeit und der Schlafeffizienzindex bei vermehrten ICU-Arousals. Sehr deutlich erkennbar ist der Zusammenhang zwischen Nachbarpatienten, Schall und ICUArousalhäufigkeit in Abbildung 3.12 (S.59). Dass Lärm den Schlaf eines Patienten negativ beeinflusst, ist ausführlich besprochen worden. Als Lärmquelle kommt zusätzlich der Nachbarpatient in Betracht. Er scheint direkt in Zusammenhang mit dem Schallpegel und der Arousalhäufigkeit des Patienten zu stehen. Offensichtlich hängt Lärm auch von der Anzahl der Nachbarn ab. Es scheint daher nahe zu liegen, dass durch getrennte Unterbringung oder Trennung vom Nachbarpatient der Schlaf eines Patienten verbessert werden könnte. Es sollte daher an eine Reduktion des Lärms durch Trennwände, Einzelzimmer oder Ähnliches gedacht werden. Auch Meyer et al. (1994), die mehrere Intensivstationen mit Mehr- und Einbettzimmer in ihrer Studie untersuchten, konnten feststellen, dass die extern induzierten Schlafstörungen in Einzelzimmern geringer sind.

Der Zusammenhang der Scores SAPSII, Ramsay- und EPS-Score mit der ICU-Arousalhäufigkeit ist komplex. Alle Scores zeigen unterschiedliche Prognosen oder Zustände der Patienten an. Der SAPS II Score beurteilt das Outcome des Patienten, die Scores Ramsay und EPS beurteilen den Ist-Zustand der Vigilanz, wobei letztgenannter zusätzlich den zu erwartenden Schallpegel in einem gegebenen Umkreis mit einschließt. Diesen komplexen Sachverhalt beachtend, ist es naheliegend, dass eine statistische Signifikanz nur schwer nachzuweisen ist.

\subsection{Resumee}

Mit dieser Untersuchung konnte eine detaillierte Aufteilung der akustischen Einflüsse auf den Patienten einer Intensivstation hergestellt werden. Es konnte nachgewiesen werden, dass insbesondere Lärm in der Lage ist, Arousals und Schlafunterbrechungen hervorzurufen. Die 
wichtigsten Schallquellen auf der untersuchten Intensivstation sind die Monitore und Perfusoren, das Personal sowie eine noch größere Menge an akustischen bis dato noch undefinierten Schallquellen. Einen erheblichen Einfluss hat zudem der Nachbarpatient. In einer nachfolgenden Untersuchung könnte das bereits besprochene Missverhältnis der ICU-Arousals zwischen 'undefinierten Lärm-Arousals' und den übrigen Arousals optimiert werden. Es könnte so eine feinere oder weitere Untergruppierung der ICU-Arousals definiert werden. Die diagnostischen Mittel sollten durch Video- und Audioüberwachung erweitert werden, um damit die Ursachen eines undefinierten Lärm-Arousals eindeutig klären zu können.

Der Vergleich mit den Ergebnissen Freedmans et al. (2001) ergab einen deutlicheren Einfluss akustischer Faktoren auf wache als auf analgosedierte und beatmungspflichtige Patienten. Im Vergleich mit dem Normkollektiv, das Carskadon und Dement (2000) sowie Steinberg et al. (2000) untersuchten, zeigte sich, dass die Patienten dieser Untersuchung durchgehend schlechter schlafen, was durchaus auf eine erhöhte Lärmbelastung und nachfolgend erhöhte Arousalaktivität zurückzuführen ist.

Aufgrund unserer Beobachtungen kann auf folgende Vorschläge hingewiesen werden, die den Schlaf eines Patienten auf einer Intensivstation verbessern könnten:

1. Schallreduktion der Alarme durch zentrales Monitoring.

- Es ist abzuwägen, ob bei jedem Patienten tatsächlich der Alarm akustisch sowohl in der Schwesternzentrale als auch in unmittelbarer Umgebung ausgelöst werden muss oder ob es nicht auch ebenso effektiv ist, den Alarm wenigstens für die Ruhezeit nur zentral akustisch auszulösen. Somit könnten insbesondere Fehlalarme ohne Störung des Patienten erkannt und beseitigt werden. Ein zentrales weiter entwickeltes Monitoring, das die Vitalparameter aller Patienten in der Schwesternzentrale zusammenfasst, wie es auf dieser ICU vorhanden war, sollte weitere Aufgaben übernehmen. Die Perfusoren und Beatmungsmaschinen z.B. könnten zusätzlich zentral gesteuert, oder zumindest überwacht werden.

2. Schallreduktion durch Einhaltung spezieller Ruhezeiten auf ICU von Seiten des Personals.

- Es wäre von Vorteil, Ruhezeiten auf ICU einzuführen, innerhalb derer das laute 
Sprechen des Personals oder helles Licht eingeschränkt wären (Notfälle und akute Intervention ausgenommen). Auch das Arousal- und Schallprofil zeigen, dass eine Verlängerung der Ruhezeiten mehr Schlaf ermöglichen könnte.

3. Verlängerung der Ruhezeiten der Patienten.

- Auch ist es erwiesen, dass längere Ruhezeiten die Schlafarchitektur verbessern. Auf der Intensivstation im Universitätsklinikum Göttingen könnten die Ruhezeiten Nachts von 4 auf 8 Stunden verlängert werden. So könnten z.B. Visiten und letzte Tätigkeiten vor der Nachtruhe bis z.B. 22 Uhr abgeschlossen sein und erste Tätigkeiten, wie z.B. Waschen, erst ab 6 Uhr beginnen. Arbeiten des Personals, der Ärzte und Schwestern sollten sich auf die restliche Zeit beschränken und nur im Notfall während der nächtlichen Ruhe durchgeführt werden. Somit hätte der Patient die Möglichkeit, länger zu ruhen und bestenfalls auch zu schlafen. Erholsamer Schlaf mit Tiefschlafstadium oder REM-Phasen ist innerhalb der Ruhezeiten am ehesten zu erwarten. So könnte auch der für die Heilung wichtige $\delta$ - oder REM-Schlaf gesteigert, und damit Schlafdeprivation und Schlafentzug vermindert werden. Außerdem wird mit der Nachtruhe der circadiane Rhythmus, der wichtig für Genesung und Gesundheit des Organismus ist, eingehalten.

4. Schallreduktion durch akustische Abschirmung - Einzelzimmer.

- Wie diese Studie gezeigt hat, leiden Patienten, die einen oder mehrere Nachbarn neben sich haben, unter störendem Lärm und Schlafunterbrechungen. Bauliche Veränderungen, z.B. Einzelzimmer, könnten hier deutlich Abhilfe schaffen. In einem Einzelzimmer genießt ein Patient Privat- und Intimsphäre. Die Tätigkeiten des Personal sowie unruhige und laute Nachbarpatienten werden in einem deutlich geringeren Maße wahrgenommen. Darüber hinaus ist eine Reduzierung des Schalllevels und der Arousals zu erwarten.

Letztlich unterstützt die Lärm- und Arousalreduktion eine schnellere Genesung. Somit ist auch mit weniger Fällen eines ICU-Syndroms zu rechnen, und die Kosten eines intensivstationären Aufenthaltes könnten durch kürzere Liegezeiten reduziert werden (Hansell 1984, Weber et al. 1985). Ein sehr interessanter und auch kostengünstiger Weg könnte die von 
Wallace et al. (1999) beschriebene Applikation von Ohrstöpseln (z.B. Oropax) an die Patienten darstellen. Wallace erreichte dadurch 1999 eine signifikante Erhöhung des REM-Anteils, wobei die REM-Latenz sank. Walder et al. (2006) stellten in der Arbeit "Schlafstörungen bei kritisch kranken Patienten " noch folgende pharmakologische und nicht-pharmakologische Therapieoptionen zusammen:

Unter pharmakologischer Behandlung versteht man die adäquate medikamentöse Schmerztherapie, sowie Angst und Stress reduzierende Sedativa, wie z.B. Barbiturate und Benzodiazepine. Alle Untersuchungen diesbezüglich bestätigten einen positiven Effekt auf die Schlafparameter, wie verlängerte TST und eine reduzierte Schlaflatenz. Nicht-pharmakologisch wurden die hier schon beschriebenen Reglements zur Reduzierung des Nachtlärms und -lichtes hervorgehoben (Siehe S.68). Außerdem wird von Walder et al. (2006) eine gewisse Evidenz im Bezug auf Musik und Massage berichtet, wobei insbesondere Musik den Schlaf verbessern und Schmerzen reduzieren soll.

Wir sehen hier mehrere sinnvolle Wege, die Schlafqualität kritisch kranker Patienten zu verbessern. Nicht jeder Vorschlag ist einfach umzusetzen, jedoch gehen wir davon aus, dass die Gesamtheit aller Bemühungen durchaus sinnvolle Ergebnisse und eine deutliche Verbesserung der Patientensituation liefern könnte. Letztlich ist es nicht nur aus ökonomischer Sicht sinnvoll und zweckmäßig, die Liegezeit zu verkürzen, sondern auch das Wohlbefinden des Patienten zu steigern und Privat- und Intimsphäre zu bieten. Zwischen dem Anspruch, den Patienten maximal zu versorgen, ihn dabei minimal zu belasten oder einzuschränken, ist eine weite Kluft. Diese gilt es durch Kompromisse, die im Einzelfall diskutiert werden müssen, zu überbrücken. 


\section{Kapitel 5}

\section{Zusammenfassung}

Wir untersuchten spontan atmende, nicht intubierte oder beatmete Patienten einer Intensivstation der Universität Göttingen mittels PSG und Soundlevelmeter. Es wurden 7 weibliche und 18 männliche Patienten einer Intensivstation, im Durchschnitt $52 \pm 15,90$ Jahre alt, untersucht und die Ergebnisse ausgewertet und analysiert.

Wir mussten feststellen, dass die Untersuchten bei einer mittleren Schlafdauer (SPT) von 6,66 Stunden 2,91 Stunden wach waren und nur 3,76 Stunden schliefen (TST), was einem Schlafeffizienzindex von nur $49 \%$ entspricht. Die Einschlaflatenz lag bei 35,4 Minuten. Die Schlafstadien Wach und S1 waren drastisch auf Kosten des $\delta$ - und REM-Schlafs erhöht. Im Mittel lag der $\delta$ - und der REM-Schlaf bei 6,02\% respektive 1,96\%. Keine der Messungen ergab ein normales Hypnogramm mit normalem REM-Schlafanteil.

Der mittlere Schallpegel mit 42,94 dB, als auch das Schallmaximum mit 85,45 DB, sowie das -minimum mit 30,39 dB waren gegenüber einer dem Schlaf adäquaten Lautstärke deutlich erhöht.

Der hohe Schallpegel bewirkte ein vermehrtes Auftreten von akustischen Arousals. 42,2\% aller Arousals waren akustischen Ursprungs. Das mit 28,3\% häufigste Arousal wurde durch undefinierten Lärm hervorgerufen. Die weiteren akustischen Arousals waren in absteigender Reihenfolge verursacht durch Monitoralarm, das Personal, den Nachbarpatienten, Perfusoralarme, Telefonklingeln und letztlich durch Alarme der Beatmungsmaschinen eines Nachbarpatienten.

Wir konnten Ruhezeiten von Aktivitätszeiten, die durch eine besonders hohe Dichte pflege- 
rischer Maßnahmen gekennzeichnet sind, abgrenzen. Während der bestehenden Ruhezeiten zwischen 0:00 Uhr und 4:00 Uhr stellten wir fest, dass die Schlafzeit und die Schlafeffizienz im Vergleich zu den Aktivitätsphasen (von 22:00 bis 0:00 Uhr, sowie von 4:00 bis 6:00 Uhr) deutlich erhöht waren, der Schallmittelwert und die ICU-Arousals hingegen auffallend vermindert waren.

Um eine Verbesserung der Schlafhygiene und eine Verminderung der Lärmbelastung auf der Intensivstation zu erreichen, fordern wir deshalb:

- Die Schallreduktion durch zentrales Monitoring.

- Die Schallreduktion durch Einhaltung und Verlängerung spezieller Ruhezeiten auf den Intensivstationen.

- Und die Schallreduktion durch akustische Abschirmung, z.B. in Einzelzimmern.

Diese Veränderungen würden für Patienten einer ICU die nötige Ruhe und Erholung gewährleisten, Privat- und Intimsphäre bieten und könnten die Heilung der Patienten beschleunigen. 


\section{Kapitel 6}

\section{Anhang}

\subsection{Abkürzungsverzeichnis}

\section{Abkürzung}

AAT

AI

ASDA

AWI

CPMP

DGSM

EAI

EEG

EOG

EPS-Score

Flow

$\mathrm{HF}$

ICU

ICU-Arousal

ICU-Syndrom

ITS

\section{Bedeutung; Synonyme}

Akustik Arousal Threshold

Arousalindex

American Sleep Disorders Association

Aufwachindex

Committee for Proprietary Medicinal Products

Deutsche Gesellschaft für Schlafmedizin

endogener Arousalindex

Elektroenzephalogramm

Elektrookulogramm

Grad des Vigilanz, der Pflegeintensität und der Schallintensität

Atemflussgeschwindigkeit

Herzfrequenz

Intensiv-Care-Unit

Intensiv-Care-Unit-Arousal

Durchgangssyndrom

Intensivstation ICU 
MT

NPPV

NREM

$\mathrm{p}$

PLMS-AI

PSG

RAI

REM

REM-Latenz

$\mathrm{RR}$

S1-S4

$\mathrm{SaO} 2$

SAPS II

SAS

SEI

SK

SOL1/SOL2

SPT

stabw

TST

Var

vs

W
Movement Time Movement-Arousal

Noninvasive Positive Pressure Ventilation

Non-Rapid-Eye-Movement

Korrelationskoeffizient

Periodic legmovement associated Arousalindex

Polysomnographie

Respiratorischer Arousalindex

Rapid-Eye-Movement

Rapid-Eye-Movement-Latenz

Blutdruck

Schlafstadium 1 bis 4

arterielle Sauerstoffsättigung

Simplified Acute Physiology Score

Schlafapnoesyndrom

Sleep Efficiency Index

Schlafkontinuität

Sleep Onset Latency

Sleep Period Time

Standardabweichung; Varianz

Total Sleep Time

Standardabweichung; Varianz stabw

versus

Wach 
KAPITEL 6. ANHANG

\subsection{Abbildungen und Tabellen}

Abbildung 6.1: Typische Schallmuster durch Monitore und Blutdruckmessgeräte verursacht

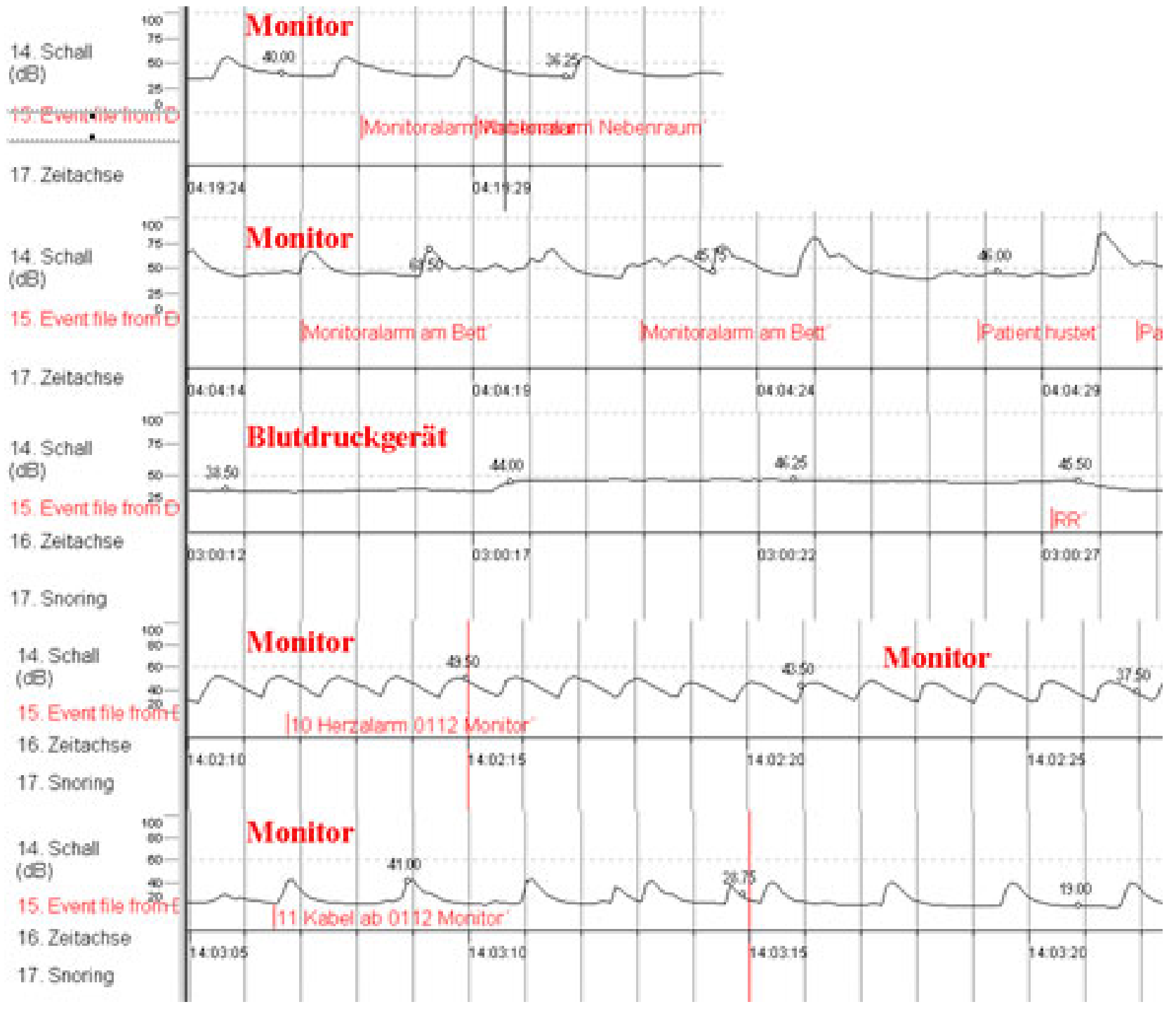

Man beachte die Regelmäßigkeit der Schallpeaks, diese sind wichtig zur Erkennung z.B. eines Monitor-Arousals. 


\section{Tabelle 6.1: Schlafparameter in der Literatur:}

\begin{tabular}{|c|c|c|}
\hline Schlafparameter & Erklärung & Tendenz \\
\hline SOL1/SOL2 & $\begin{array}{l}\text { Einschlaflatenz vom zu Bett } \\
\text { gehen bis erstes S1/S2 }\end{array}$ & $\Uparrow$ \\
\hline $\mathrm{SPT}$ & Schlafperiodendauer & $\Downarrow$ \\
\hline TST & Totale Schlafzeit & $\Downarrow$ \\
\hline SEI & Schlaf Effizienzindex & $\Downarrow$ \\
\hline SK & Schlafkontinuität & $\Downarrow$ \\
\hline NREM S1 & & $\Uparrow$ \\
\hline NREM S2 & & $\Uparrow$ \\
\hline$\delta$-Schlaf & NREM S3 und S4 & $\Downarrow$ \\
\hline REM & & $\Downarrow$ \\
\hline REM-Latenzzeit & $\begin{array}{l}\text { Zeit bis zum ersten Auftre- } \\
\text { ten REM }\end{array}$ & $\Uparrow$ \\
\hline Arousals & & $\Uparrow$ \\
\hline $\begin{array}{l}\text { Arousal mit folgender } \\
\text { Wachphase }\end{array}$ & & $\Uparrow$ \\
\hline Schlafstadienwechsel & & $\Uparrow$ \\
\hline Schlaffragmentierung & & $\Uparrow$ \\
\hline Schlaf unter Tags & & $\Uparrow$ \\
\hline
\end{tabular}

Tendenz der Schlafparameter eines Intensivpatienten verglichen mit denen eines Gesunden. $\Uparrow:$ erhöht oder; $\Downarrow$ : erniedrigt 
Tabelle 6.2: Kanalkonfiguration der Aufzeichnung und der Anzeige

\begin{tabular}{l|c|c|r} 
Kanal & Signalcode & Filter $(\mathrm{Hz})$ & Abtastrate $(\mathrm{Hz})$ \\
\hline Die Kanäle wurden in folgender Konfiguration abgetastet, gefiltert und aufgenommen \\
EEG1 & C3-A2 & $0,1-35$ & 98,9188 \\
EEG2 & C4-A2 & $0,1-35$ & 98,9188 \\
EOG1 & ROC-A2 & $0,5-35$ & 98,9188 \\
EOG2 & LOC-A2 & $0,5-35$ & 98,9188 \\
EMG1 Kinn & EMG1 \pm & $0,03-70$ & 98,9188 \\
EMG2 Bein links & EMG2 \pm & $0,03-70$ & 98,9188 \\
EMG3 Bein rechts & EMG3 \pm & $0,03-70$ & 98,9188 \\
Flow & Rsp1 \pm & $0,5-5$ & 16,485 \\
Thoraxsensor & Rsp2 \pm & $0,5-5$ & 16,485 \\
Abdomensensor & Rsp3 \pm & $0,5-5$ & 16,485 \\
Schnarch & Rsp4 \pm & $30-70$ & 16,485 \\
SaO 2 (Sättigung) & X2 & $0-3$ & 5,75109 \\
HF (Herzfrequenz) & X3 & $0-3$ & 5,75109 \\
EKG & O1/O2 & $0,05-120$ & 197,838 \\
Schallpegel & X5 & DC & 10,752 \\
\hline Die Kan & &
\end{tabular}

Die Kanäle kamen in folgender Konfiguration abgetastet, gefiltert zur Anzeige

\begin{tabular}{l|c|c|c} 
EEG1 & EEG & $0,03-35$ & 98,9188 \\
EEG2 & EEG & $0,03-35$ & 98,9188 \\
EOG1 & EOG & $0,05-70$ & 98,9188 \\
EOG2 & EOG & $0,05-70$ & 98,9188 \\
EMG1 Kinn & EMG & $1-200$ & 98,9188 \\
EMG2 Bein links & EMG & $1-200$ & 98,9188 \\
EMG3 Bein rechts & EMG & $1-200$ & 98,9188 \\
Flow & RSP & $0,03-15$ & 16,4865 \\
Thoraxsensor & RSP & $0,03-15$ & 16,4865 \\
Abdomensensor & RSP & $0,03-15$ & 16,4865 \\
Schnarch & RSP & $5-200$ & 164,865 \\
SaO ${ }_{2}$ (Sättigung) & SaO 2 & $0-0$ & 5,75109 \\
HF (Herzfrequenz) & DC & $0-3$ & 5,75109 \\
EKG & DC & $0,03-400$ & 197,838 \\
Schallpegel & DC & $0-0$ & 10,752
\end{tabular}

RSP: Respiratorisch; DC: Kanäle externer Geräte. 


\section{Liste 6.1 Standardereignisse:}

- Alarme

- Perfusoralarm am Bett

- Perfusoralarm am Nachbar

- Perfusoralarm Nebenraum

- Tropfenzähleralarm

- Monitoralarm am Bett

- Monitoralarm am Nachbar

- Monitoralarm Nebenraum

- Beatmungsgerät

- Beatmungsgerät Nachbar

- Beatmungsgerät Nebenraum

- RR Messung

- Manipulation

- Arztvisite

- Absaugen

- Schwester/Pfleger -Tätigkeit am Bett

- Kabel ab

- Kabel angeschlossen

- Kabel erneuert

- Patient Eigengeräusche

- Husten

- Schnarchen

- Keuchen

- Niesen

- Bewegung
- Patient möchte Schmerzmittel

- Patient möchte Schlafmittel

- Patient ruft/schreit

- Patient hustet

- Andere Geräusche

- Schwester/ Pfleger -Tätigkeit im Zimmer

- Schwester/ Pfleger -Tätigkeit Nebenraum

- Neuzugang im Zimmer

- Neuzugang im Nebenraum

- Nachbarpatient ruft/ schreit

- Nachbarpatient hustet

- Nachbarpatient wird abgesaugt

- Sonstiges

- Beleuchtung an

- Beleuchtung aus

- Patient wach

- Telefon 
Tabelle 6.3: Patientenübersicht

\begin{tabular}{|c|c|c|c|c|c|c|c|c|c|c|}
\hline Pat & Alt & Sex & $\mathrm{Na}$ & EPS & Ram & ICU & BDK & PCA & Grund & Nachtmed \\
\hline $\mathrm{A}$ & 50 & $\mathrm{w}$ & 2 & 3 & 2 & 1 & ja & nein & Abd. OP & keine \\
\hline B & 20 & $\mathrm{w}$ & 1 & 2 & 2 & 1 & ja & ja & Orth. & keine \\
\hline $\mathrm{C}$ & 43 & $\mathrm{~m}$ & 0 & 1 & 1 & 6 & nein & ja & Thor. OP & keine \\
\hline $\mathrm{D}$ & 60 & $\mathrm{~m}$ & 1 & 1 & 2 & 1 & ja & nein & Kard. OP & keine \\
\hline $\mathrm{E}$ & 50 & $\mathrm{~m}$ & 2 & 2 & 2 & 1 & ja & nein & IN & keine \\
\hline $\mathrm{F}$ & 29 & $\mathrm{w}$ & 0 & 1 & 2 & 1 & nein & nein & Gyn. OP & keine \\
\hline G & 32 & $\mathrm{w}$ & 3 & 2 & 2 & 1 & nein & nein & Thor. OP & keine \\
\hline H1a & 71 & $\mathrm{w}$ & 2 & 2 & 2 & 1 & ja & nein & Orth. & keine \\
\hline I & 74 & $\mathrm{w}$ & 1 & 1 & 2 & 2 & nein & nein & Kard. OP & keine \\
\hline $\mathrm{J} 2 \mathrm{a}$ & 59 & $\mathrm{~m}$ & 1 & 1 & 2 & 5 & nein & nein & Trauma & keine \\
\hline K & 56 & $\mathrm{~m}$ & 3 & 3 & 2 & 1 & ja & nein & Abd. OP & keine \\
\hline $\mathrm{L}$ & 44 & $\mathrm{~m}$ & 1 & 3 & 2 & 1 & nein & nein & Orth. & Dipidolor \\
\hline M & 70 & $\mathrm{~m}$ & 2 & 2 & 2 & 1 & ja & nein & Abd. OP & Stangyl Tr. \\
\hline N3b & 76 & $\mathrm{~m}$ & 0 & 1 & 2 & 3 & ja & nein & Abd. OP & Rem. Dia. \\
\hline O4a & 50 & $\mathrm{~m}$ & 1 & 2 & 1 & 7 & ja & nein & Uro & keine \\
\hline $\mathrm{P}$ & 65 & $\mathrm{~m}$ & 0 & 1 & 1 & 3 & ja & nein & Abd. OP & keine \\
\hline $\mathrm{Q}$ & 59 & $\mathrm{~m}$ & 2 & 2 & 2 & 1 & ja & nein & HNO & Remestan \\
\hline R5a & 60 & $\mathrm{~m}$ & 3 & 1 & 1 & 2 & ja & nein & Abd. OP & keine \\
\hline S5b & 60 & $\mathrm{~m}$ & 3 & 2 & 2 & 3 & ja & nein & Abd. OP & keine \\
\hline T6a & 56 & $\mathrm{~m}$ & 0 & 1 & 2 & 3 & nein & nein & Trauma & keine \\
\hline U6b & 56 & $\mathrm{~m}$ & 0 & 1 & 2 & 4 & nein & nein & Trauma & keine \\
\hline V7a & 28 & $\mathrm{~m}$ & 1 & 2 & 2 & 2 & ja & nein & Trauma & keine \\
\hline W7b & 28 & $\mathrm{~m}$ & 2 & 2 & 2 & 3 & nein & ja & Trauma & keine \\
\hline $\mathrm{X}$ & 36 & $\mathrm{~m}$ & 1 & 2 & 2 & 8 & $\mathrm{Ja}$ & Nein & Trauma & keine \\
\hline $\mathrm{Y}$ & 70 & $\mathrm{w}$ & 0 & 1 & 2 & 4 & ja & nein & HNO & keine \\
\hline
\end{tabular}

Übersicht der Population aller gültigen Messungen.

Pat: Patienten Nummer; alt: Alter; sex: Geschlecht; Na: Anzahl der Nachbarn; EPS: EPS-Score; Ram: Ramsay Score; ICU-Tag: Tag auf Intensivstation; BDK: Blasendauerkatheter: PCA: patient controlled Analgesia; Grund: Grund der Aufnahme; Nachtmed: Nachtmedikation;

Abd. OP: Abdominalchirurgisch; Gyn.: Gynäkologisch ; HNO: Hals-Nasen-OhrenKunde; Kard. OP: Kardiologische Operation; Orth: Orthopädisch; IN : Internistisch; Thor. OP: Thorakalchirurgisch; Uro: Urologisch; Trauma: Traumatologisch;

Rem. - Remestan; Dia. - Diazepam; 


\section{Literaturverzeichnis}

[Aaron et al. 1996] Aaron JN, Carlisle CC, Carskadon MA, Meyer TJ, Hill NS, Millman RP (1996): Environmental Noise as a Cause of Sleep Disruption in an Intermediate Respiratory Care Unit. Sleep, 19(9), 707-710

[ASDA 1997] American Sleep Disorders Association: the international classification of sleep disorders, revised diagnostic and coding manual, American Academy of Sleep Medicine. One Westbrook Corporate Center, Westchester, IL, USA 1997

[Aurell und Elmqvist 1985] Aurell J, Elmqvist D (1985): Sleep in the surgical intensive care unit: continuous polygraphic recording of sleep in nine patients receiving postoperative care. Br Med J, 290, 1029-1032

[Bentley et al. 1977] Bentley S, Murphy F, Dudley H (1977): Perceived noise in surgical wards and an intensive care area: an objective analysis. Br Med J, 1977/2, 1503-6

[Brockhaus 2004] Brockhaus: Der Brockhaus Bd.4 Bibliographisches Institut \& F. A. Brockhaus AG Leipzig 2004

[Calvete Vazquez et al. 2000] Calvete Vazquez R, Garcia Arufe MB, Uriel Latorre P, Fernandez Lopez V, Medin Catoira B (2000): Patients' sleep in intensive care units and sleep-modifying factors. [Article in Spanish], Enferm Intensiva, $11(1) 10-16$ 
[Carola et al. 1990] Carola R, Harley J, Noback CR: Human anatomy and physiology. McGraw Hill, New York 1990, 73-77

[Carskadon und Dement 2000] Carskadon M, Dement W: Normal human sleep: an overview. In: Principles and practice of sleep medicine. Bd. 3, Harcourt Brace Jovanovich, Philadelphia, USA 2000, 15-25

[CPMP 1990] CPMP Working Party EEC note for guidance (1990): good clinical practice for trials on medicinal products in the European Community. on Efficacy of Medicinal Products. Pharmacol Toxicol, 67(4), 361-72

[De Jonghe et al. 2000] De Jonghe B, Cook D, Appere-De-Vecchi C, Guyatt G, Meade M, Outin H (2000): Using and understanding sedation scoring systems: a systematic review. Intensive Care Med, 26(3), 275-285

[Dement und Kleitman 1957a] Dement W, Kleitman N (1957): Cyclic variations in EEG during sleep and their relation to eye movements, body motility and dreaming. Electroencephalogr Clin Neurophysiol, $\underline{9}, 673-690$

[Dement und Kleitman 1957b] Dement W, Kleitman N (1957): The relation of eye movements during sleep to dream activity: an objective method of the study of dreaming. J Exp Psychol, $\underline{53}, 339-46$

[Dyer et al. 1995] Dyer CB, Ashton CM, Teasdale TA (1995): Postoperative delirium. A review of 80 primary data-collection studies. Arch Intern Med., 155(5), 461-5

[Edwards und Schuring 1993] Edwards GB, LM Schuring (1993):Pilot study: validating staff nurses' observations of sleep and wake states among critically ill patientes, using polysomnography. Am J Crit Care, 2.2, 125-31

[Ferrara et al. 1999] Ferrara M, De Gennaro L, Casagrande M, Bertini M (1999): Auditory arousal thresholds after selective slow-wave sleep deprivation. Clin Neurophysiol, 110(12), 2148-52 
[Freedman et al. 1999] Freedman N, Kotzer N, Schwab R (1999): Patient Perception of Sleep Quality and Etiology of Sleep Disruption in the Intensiv Care Unit. Am J Crit Care, 159(4 Pt 1), 1155-1162

[Freedman et al. 2001] Freedman N, Gazendam J, Levan L, Pack A, Schwab RJ (2001): Abnormal sleep/wake cycles and the effect of environmental noise on sleep disruption in the intensive care unit. Am J Respir Crit Care Med, 163(2), $451-457$

[Hansell 1984] Hansell HN (1984): The behavioral effects of noise on man: the patient with "intensive care unit psychosis". Heart Lung, 13(1), 59-65

[Helton et al. 1980] Helton MC, Gordon SH, Nunnery SL (1980): The correlation between sleep deprivation and the intensive care unit syndrome. Heart Lung, $\underline{9(3)}, 464-468$

[Hobson 1989] Hobson JA (1989): Sleep. Scientific American Library, New York 1989

[Jasper 1958] Jasper HH (1958): The ten-twenty electrode system of the International Federation. Electroenephalogr Clin Neurophysiol 10, 371-375

[Johns et al. 1974] Johns MW, Large AA, Masterton JP, Dudley HAF (1974): Sleep and delirium after open heart surgery. Br J Surg, $\underline{61}, 377-381$

[Krachman und Criner 2002] Krachman SL, GJ Criner (2002): Sleep and long-term ventilation. Respir Care Clin N Am, 8(4), 611-29

[Krachman et al. 1995] Krachman SL, GE D'Alonzo, GJ Criner (1995): Sleep in the intensiv care unit. Chest, 105(4), 1713-20

[Le Gall et al. 1993] Le Gall JR, S Lemeshow, F Saulnier (1993): A new Simplified Acute Physiology Score (SAPS II) based on a European/North Amerikan mulficenter study. JAMA, 270, 2957-63 
[Loomis et al. 1935] Loomis AL, Harvey EN, Hobart GA (1935): Potential rhythms of the cortex during sleep. Science, $\underline{81}, 597-598$

[Loomis et al. 1937] Loomis AL, Harvey EN, Hobart GA (1937): Cerebral states during sleep, as studied by human brain potentials. J Exp Psychol, 21, 127-144

[McGuire et al. 2000] McGuire BE, Basten CJ, Ryan CJ, Gallagher J (2000): Intensive care unit syndrome- A dangerous misnomer. Arch Intern Med, 160(7), 906-909

[Meyer et al. 1994] Meyer TJ, SE Eveloff, MS Bauer, WA Schwartz, NS Hill, RP Millman (1994): Adverse environmental conditions in the respiratory and medical ICU settings. Chest, 105(4), 1211-16

[Olson et al. 2001] Olson DM, Borel CO, Laskowitz DT, Moore DT, McConnell ES (2001): Quiet time: A nursing intervention to promote sleep in neurocritical care units. Am J Crit Care, 10(2), 74-78

[Penzel et al. 1993] Penzel T, Hajak G, Hoffmann RM (1993): Empfehlungen zur Durchführung und Auswertung polygraphischer Ableitungen im diagnostischen Schlaflabor. EEG EMG Z Elektroenzephalogr Elektromyogr Verwandte Geb, $\underline{24}$, 65-70

[Ramsay et al. 1974] Ramsay MA, Savege TM, Simpson BR, Goodwin R. (1974): Controlled Sedation with Alphaxalone-Alphadolone. Br Med J, 1974/2, 656659

[Rechtschaffen und Kales 1968] Rechtschaffen A, Kales A: A Manual for Standardized Terminology, Techniques and Scoring Systems for Sleep Stages of Human Subjects, Public Health Service US Government Printing Office, Washington DC 1968, 1-56

[Redeker 2000] Redeker, NS (2000): Sleep in acute care settings: an integrative review. J Nurs Scholarsh 32(1), 31-38 
[Rosenberg et al. 1995] Rosenberg J, Rosenberg-Adamsen S, Kehlet H (1995): Postoperative sleep disturbance: causes, factors and effects on outcome. Eur J Anaesthesiol Suppl, 12(10), 28-30

[Sanner 1992] Sanner B (1992): Welche Bedeutung hat das obstruktive Schlafapnoesyndrom postoperativ oder auf Intensivstationen? Dtsch Med Wochenschr $\underline{117}, 1122-1123$

[Schwab 1994] Schwab RJ (1994): Disturbances of sleep in the intensive care unit. Crit Care Clin, 10(4), 681-694

[Sebilia 1981] Sebilia A, RN, MSN, CCRN (1981): Sleep Deprivation and Biological Rhythms in the Critical Care Unit. Crit Care Nurse, 1(4) 19-23

[Steinberg et al. 2000] Steinberg R, HG Weeß, R Landwehr: Schlafmedizin: Grundlagen und Praxis, UNI-MED Verlag Bremen 2000

[Topf 1992] Topf M (1992): Effects of Personal Control over Hospital Noise on Sleep. Res Nurs Health, 15(1), 19-28

[Topf et al. 1995] Topf M, Bookman M, Arand D (1995): Effects of critical care unit noise on the subjective quality of sleep. J Adv Nurs, $\underline{24(3)}$, 545-551

[Walder et al. 2000] Walder B, Francioli D, Meyer J, Lançon M, Romand J (2000): Effects of guidelines implementation in a surgical intensive care unit to control nighttime light and noise levels. Crit Care Med, 28(7), 2242-2247

[Walder et al. 2006] Walder B, Haase U, Rundshagen I: Schlafstörungen bei kritisch kranken Patienten, Der Anästhesist, (Online Publikation 2006), Springer Medizin Verlag, Berlin 2006

[Wallace et al. 1999] Wallace J, Robins J, Alvord L, Walker J (1999): The effect of earplugs on Sleep Measures During Exposure to Simulated Intensive Care Unit Noise. Am J Crit Care, 8(4), 210-219 
[Weber et al. 1985] Weber RJ, Oszko MA, Bolender BJ, Grysiak DL (1985): The intensive care unit syndrome: causes, treatment, and prevention. Drug Intell Clin Pharm, 19(1), 13-20

[Wood 1993] Wood A (1993): A review of literature relating to sleep in hospital with emphasis on the sleep of the ICU patient. Intensive Crit Care Nurs, $\underline{9}$, 129-136

[Woodward et al. 2000] Woodward SH, Murburg MM, Bliwise DL (2000): PTSDrelated hyperarousal assessed during sleep. Physiol Behav, 70(1-2), 197203 


\section{Abbildungsverzeichnis}

1.1 Verteilungsmuster der Schlafstadien eines Gesunden nach Carskadon und Dement (2000) sowie Steinberg et al. (2000) . . . . . . . . 5

1.2 Schall und Arousal . . . . . . . . . . . . . . . . . . . . . . 13

2.1 Lageplan der Intensivstation der Universität Göttingen 0112 . 19

2.2 Die Platzierung der Elektroden nach dem internationalen 10/20System (Jasper 1958) f . . . . . . . . . . . . . . . . . . . . . 22

2.3 Versuchsaufbau . . . . . . . . . . . . . . . . . 25

3.1 Altersverteilung aller gemessenen Patienten . . . . . . . . . . . . 41

3.2 Überblick Nachbarpatienten . . . . . . . . . . . . . . . . . . . . . 42

3.3 Die Normalverteilung der Schlafparameter . . . . . . . . . . . . 44

3.4 Schlafstadienverteilung im Überblick . . . . . . . . . . . . . . . 45

3.5 Alle Arousals im Überblick . . . . . . . . . . . . . . . . . . . . . . 49

3.6 ICU- und Standard-Arousals . . . . . . . . . . . . . . . . . 50

3.7 Zeit vs Schallmittelwert -maximum und -minimum . . . . . . 53

3.8 Zeit vs Standard- und ICU-Arousalindex . . . . . . . . . . . . 54

3.9 Zeit vs AWI und TST . . . . . . . . . . . . . . . . . 55

3.10 Anteil der ICU-Arousals versus TST und Ramsay . . . . . . . . 57

3.11 Anteil der ICU-Arousals versus SEI . . . . . . . . . . . . 58

3.12 Nachbarpatient vs Schallmittelwert und Anteil der ICU-Arousals an Gesamt . . . . . . . . . . . . . . . . . . . . . 59 
4.1 Graphische Gegenüberstellung der Normwerte von Carskadon und Dement (2000) sowie Steinberg et al. (2000) . . . . . . . 70

4.2 Hypnogramm eines Gesunden im Vergleich mit pathologischem Hypnogramm . . . . . . . . . . . . . . . . 71

6.1 Typische Schallmuster durch Monitore und Blutdruckmessgeräte verursacht $\ldots \ldots \ldots \ldots \ldots \ldots \ldots$ 


\section{Tabellenverzeichnis}

1.1 Zusammenfassende Definition der Standard-Arousals nach Steinberg et al. 2000 (S.44-55 und S.145-148) . . . . . . . . . . . 8

1.2 Intrinsische und extrinsische Faktoren auf ICU . . . . . . . . . . 11

2.1 Ramsay-Score: die 6 Grade der Sedierung eines Patienten (Ramsay et al. 1974) . . . . . . . . . . . . . . . . . . 26

2.2 Die Kategorien des EPS-Score: . . . . . . . . . . . . . . . . . . . . 27

2.3 Schlafparameter zur Qualifizierung und Quantifizierung des Schlafs nach Steinberg et al. (2000) (S.146) . . . . . . . . . . . . . . 30

2.4 Die Arousal-Kriterien der ASDA zusammengefasst von Steinberg et al. (2000) nach den internationalen Kriterien von Rechtschaffen und Kales (1968) und den Richtlinien der DGSM (Penzel et al. 1993) 31

2.5 Die Kriterien der ICU-Arousals: . . . . . . . . . . . . . . . . . . . 33

2.6 Definition ICU-Arousal . . . . . . . . . . . . . . . . . . . . . 34

2.7 "Normal human sleep" nach Carskadon und Dement 2000 (S.20) 36

2.8 Akustische und non-akustische Arousals . . . . . . . . . . . . . . 37

3.1 Ramsay- und EPS-Score . . . . . . . . . . . . . . . . . . 42

3.2 Ergebnisse der Schlafparameter . . . . . . . . . . . . . 43

3.3 Schlafparameter aller Messungen A . . . . . . . . . . . . . 46

3.4 Schlafparameter aller Messungen B . . . . . . . . . . . . . 47

3.5 Ergebnisse der Schallmessung . . . . . . . . . . . . . . . 48

3.6 Arousals akustisch - nicht-akustisch . . . . . . . . . . . . 51 
3.7 Korrelation des Anteils der ICU-Arousals . . . . . . . . . . 56

4.1 Orientierende Kennwerte nach Steinberg et al. (2000) _ . . . 66

4.2 Vergleich mit den Parametern von Freedman et al. (2001) . . . 74

6.1 Schlafparameter in der Literatur: . . . . . . . . . . . . . . 85

6.2 Kanalkonfiguration der Aufzeichnung und der Anzeige . . . . 86

6.3 Patientenübersicht . . . . . . . . . . . . . . . . . . . . 88 


\section{Danksagung}

Ich möchte mich ganz herzlich bei meinem Doktorvater, Herrn Prof.Dr. H.Burchardi, der die Untersuchung ermöglichte und dieser Dissertation Form und Stil verlieh, bedanken. Besonderer Dank gilt meinen Betreuern Dr. Tim Deegener und Dr. Thomas Schneider, deren Engagement, Wissen und Ausdauer den Anstoß und die Durchführung dieser Untersuchung ermöglichten, die die Untersuchung leiteten und zur Vollendung

brachten. Ich danke den Ärzten und Schwestern der Stationen 0112 und 0113 für deren Mit- und Zusammenarbeit, sowie allen anderweitig Beteiligten und Mitwirkenden. 


\section{Lebenslauf}

Ich wurde am 24.01.1975 in Memmingen geboren. Ich habe das Abitur am Gymnasium mit Schülerwohnheim Hohenschwangau 1995 absolviert, worauf ich den einjährigen Zivildienst als Stationshilfe an der Fachklinik für orthopädische und neurologische Rehabilitation Enzensberg, Hopfen am See, leistete.

Das Medizinstudium begann ich im Wintersemester des Jahres 1996 an der GeorgAugust-Universität Göttingen. Begleitend studierte ich Sport von 1997 bis 2002 und begann 2000 im Zentrum für Anästhesiologie bei Prof. Dr. Burchardi zu promovieren. Meine Zeit in Göttingen beendete ich 2002 mit dem 2. Staatsexamen der Medizin und setzte das Praktische Jahr am Klinikum Bietigheim-Bissingen mit abschließendem 3. Staatsexamen an der Ruprecht-Karls-Universität Heidelberg fort. Den 'Arzt im Praktikum' begann ich ebenfalls im Klinikum Bietigheim-Bissingen unter Chefarzt Dr. Thomas Vögele in der Abteilung Anästhesie, Schmerztherapie und Intensivmedizin, und erhielt im Oktober 2004 meine Approbation als Arzt.

Die Anstellung behielt ich bis März 2005, um dann in die Chirurgie am Klinikum Günzburg zu wechseln. Seit Juli 2006 arbeite ich regelmäßig als Notarzt im Landkreis Günzburg. Im September 2007 wechselte ich für ein knappes Jahr ins Klinikum Heidenheim in die Thorax-, Viszeral- und Gefäßchirurgie unter der Leitung von Prof. Dr. A. Imdahl. Seit Juli 2008 bin ich wieder in der Chirurgie im Klinikum Günzburg unter der chefärztlichen Leitung von Dr. D. Michel, Dr. U. Widmaier und Dr. U.Kugelmann bis heute als Assistenzarzt tätig.

Am 25.01.08 erblickte meine Tochter Ronja Maria Barbara das Licht der Welt und am 28.12.2009 ging ich mit meiner Frau Manuela Engels, geborene Schott, den Bund der Ehe ein. 